\title{
Forces and Variational Compatibility for Equilibrium Liquid Crystal Director Models with Coupled Electric Fields
}

\author{
Eugene C. Gartland, Jr.
}

September 24, 2019

\begin{abstract}
Expressions are obtained for force and couple densities and stress tensors in macroscopic models for nematic liquid crystals subjected to electric fields. The coupling between the liquid crystal orientational properties and the electric field is taken into account via a free energy of Oseen-Frank type expressed as an integral functional of the director field and the electric potential field. The variational model here also allows for a gravitational field and a magnetic field, and the differences among these three common types of force fields (gravitational, magnetic, and electric) are discussed. Also included in the free energy is a surface anchoring potential, and its effect on boundary traction and couple stress is explored. The electric field is assumed to arise from electrodes held at constant potential. Flexoelectric effects are included, and as a consequence, the material is no longer a linear dielectric medium. It is shown that the equilibrium solutions of the Euler-Lagrange equations satisfy appropriate expressions of force balance and torque balance, obtained from a virtual-work principle, and a connection is made between this and ideas related to Noether's Theorem. The development here builds from theories of Ericksen related to the notion of "variational compatibility," and this connection is made. Comparisons are also made to the extensive literature in physics and continuum mechanics on electromagnetic field/matter interaction.
\end{abstract}

Keywords liquid crystal · Oseen-Frank model · surface anchoring energy · electric field · flexoelectricity $\cdot$ stress tensor

PACS 61.30.-v $\cdot 61.30 . \mathrm{Dk} \cdot 61.30 . \mathrm{Gd} \cdot 61.30 . \mathrm{Hn} \cdot 77.84 . \mathrm{Nh}$

\section{Introduction}

Our interest is in macroscopic continuum models for the orientational properties of materials in a liquid crystal phase, a complex partially ordered fluid phase exhibited by certain materials in certain parameter ranges. Such models are used at the scales of typical devices and experiments involving these kinds of materials. Our primary objective is to obtain

E. C. Gartland, Jr.

Department of Mathematical Sciences, Kent State University, Kent, Ohio 44242, USA

Tel.: +1-330-672-9112, Fax:+1-330-672-2209, E-mail: gartland@math.kent.edu 
expressions for the various stress tensors, couple stress tensors, and boundary tractions in situations in which the material is subjected to an electric field. These tensors are required to describe conservation laws in the hydrodynamic theory, and they are sometimes needed to model experiments. Electric fields have been used for decades to control liquid crystals, and treating these fields as analogous to the better-understood magnetic fields often produces adequate approximations. There are, however, aspects to the coupling between electric fields and liquid-crystal orientational properties that have lacked complete understanding, especially with respect to forces and stresses, and especially with respect to situations in which flexoelectric polarization needs to be taken into account.

The history of the macroscopic continuum theory of liquid crystals has its origins in the works of Oseen [48], Zocher [65], and Frank [22]. It was brought into the realm of modern continuum mechanics by Ericksen, who together with Leslie developed the hydrodynamic theory (the Ericksen-Leslie equations). The review articles [15] and [41] contain numerous references. The standard modern reference in the physics literature is [24]; while the standard references in the applied mathematics and continuum mechanics literature are [55,56, 63]. At this level of modeling, the orientational state of the material is characterized by a unit-length vector field $\boldsymbol{n}$ (referred to as the "director field"), which represents the average orientation of the distinguished axis of the anisometric molecules in a fluid element at a point. Central to the modeling of both equilibria and dynamics is an appropriate expression for the free energy of the system, which serves as a work function (stored-energy function) for isothermal, reversible processes. In the models of interest to us, the material is also treated as incompressible.

Liquid crystals are very responsive to external stimuli, such as magnetic fields and electric fields, and this has been one of the keys to their usefulness in technological applications. Ericksen deliberately ignored this aspect in his first papers, as he focused on other features of liquid-crystal systems, such as their stress tensors, which are not necessarily symmetricthese are examples of what are known as "polar materials" [61, Sec. 98]. Ericksen finally sought to introduce external fields into his development in [14], where he presented sufficient conditions for the director field that solved the equilibrium Euler-Lagrange equations associated with the free energy to be consistent with the hydrostatic limit of the EricksenLeslie equations in the presence of external force fields, an issue which he termed "variational compatibility." We discuss the details of these ideas in Sec.3. They work well for systems involving gravitational and/or magnetic fields, and this is the accepted approach in those settings. The additional complications associated with electric fields, however, have been acknowledged for a long time-quoting Leslie [41, Sec. B.4]: "Although there are rather obvious dielectric analogies for electric fields, we do not discuss this further on account of other complications that can occur in such cases." Similar cautionary notes can be found in [15. Sec. II.A.1] and [56. Secs. 3.5, 4.2.4], while the discussion of the hydrostatics of nematics in [24, Sec. 3.5.1] explicitly avoids electric fields and flexoelectric effects.

Three types of force fields of external origin are commonly discussed in the context of liquid crystals: gravitational fields, magnetic fields, and electric fields. Gravitational fields seldom have enough influence to warrant inclusion in a model, although there are exceptions (see [15. Sec. II.A.1]). Magnetic fields are commonly used in experiments, sometimes in combination with electric fields. Electric fields are very common in devices, as well as in experiments. Gravitational fields are true external fields, i.e., independent of the state of the material at a point [27, Sec. 9.01]. Magnetic fields are influenced by liquid crystal materials, which are anisotropic with magnetic susceptibilities that depend on the orientational state of the material at a point. For the parameter values of typical liquid-crystal materials, however, this influence is negligible—we elaborate below in Sec.2.1. Thus a magnetic field in a liquid 
crystal can be treated as an external field. An electric field is influenced by the state of the liquid-crystal material in a similar fashion; however the coupling is much stronger and cannot be ignored-this also is quantified below. In the development here, we consider all three types of force fields, and we will see how they contribute differently to the body forces, stresses, body couples, couple stresses, and tractions.

The literature on electromagnetic fields in matter is vast and contains more than its share of controversies. In the physics literature, one can find decades-long debates about the appropriate expressions for electromagnetic momentum and stress in dielectrics, a prominent example being the "Abraham-Minkowski controversy"-for a contemporary view, see [4,5. 7] and references contained therein. In the continuum-mechanics literature, there has been extensive development in the areas of electromagnetics in deformable and fluent matter, tracing back to Toupin [59] and continuing to this day. This work has been motivated by a variety of applications, including piezoelectricity, photoelasticity, electro/magneto-active polymers, electrogels, electro-rheological fluids, and the like. The models take a variety of forms, due to several factors: different application domains, no unique choice of basic electromagnetic variables, no unique definition of electromagnetic stress and body force, and the need to postulate expressions for such quantities. More recent papers and books have illustrated the sameness of several different-looking models—-see for example [9, 10, 43] and [32, Ch. 4]. We shall make contact with this literature at points in our development.

Our setting here is simpler in several ways (isothermal, incompressible, equilibrium, non-optical fields); yet it is more complicated in others (inhomogeneous, anisotropic, nonlinear dielectric medium). A major advantage that we enjoy is that we are able to work from an expression for the free energy that is of widely accepted form, from which we can derive the quantities of interest, thereby avoiding the postulational approach and associated controversies. Our approach is to employ a virtual work principle as used by Ericksen in his original work [13, Sec. IV], as recounted in [56, Sec. 2.4.2] and [61, Secs. 127-8], except that here we will take into account the coupling between the director field and the electric field. The approach is similar in spirit to that found in [40, Sec. 15], [57, Sec. 2.21], and [59, Sec. 10].

The paper is organized as follows. In Section 2 we introduce the variational model, including all fields and terms that contribute to the free-energy density and surface anchoring energy. Section 3 concerns the relationship between the variational equilibrium equations and the hydrostatic limit of the Ericksen-Leslie flow equations. There the issue of "variational compatibility" and Ericksen's "compatibility potential" are introduced. Expressions for the balance of forces and balance of torques that characterize hydrostatic equilibrium are established in Sec.4. Also introduced in that section is a virtual-work principle, from which expressions are derived for the stress tensor, couple stress tensor, and boundary conditions for our model. Section 5 contains an attempt to interpret our results and to put them in context with related results in the literature. The final Sec.6 summarizes our main findings.

\section{Model Problem}

We are interested in the modeling of liquid-crystal systems as found in typical experiments with such materials: a thin-film liquid-crystal cell sandwiched between a combination of glass substrates, alignment layers, optical components, and conducting layers that are attached to a variable voltage source. The typical setup is, in essence, that of a parallel-plate capacitor with a complex, changeable dielectric_changes in the voltage cause changes in the orientational state of the liquid crystal, which in turn cause changes in the effective ca- 


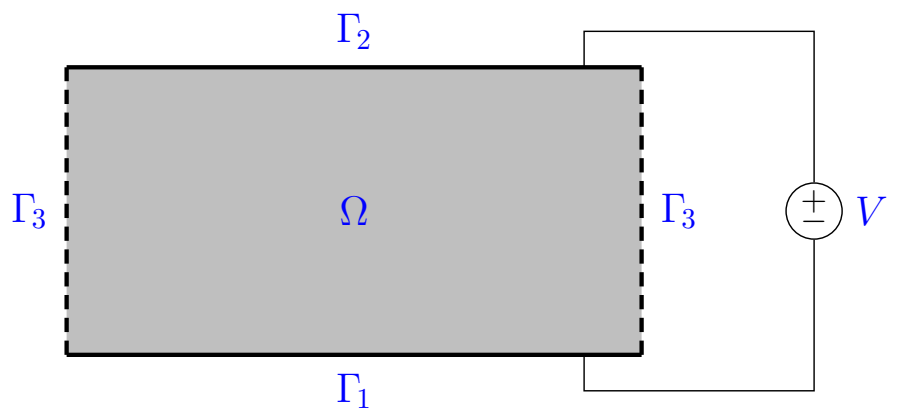

Fig. 1 Model problem domain (two-dimensional depiction of actual three-dimensional, hexahedral domain). Boundary conditions: on $\Gamma_{1}$ (strong anchoring, Dirichlet boundary condition $\boldsymbol{n}=\boldsymbol{n}_{0}$ on $\boldsymbol{n}, U=-V / 2$ ), on $\Gamma_{2}$ (weak anchoring, natural boundary condition $\left[11 \mathrm{~b}\right.$ on $n, U=V / 2$ ), on $\Gamma_{3}$ (periodic boundary conditions on both $\boldsymbol{n}$ and $U$ on opposing sides of $\Gamma_{3}$ ).

pacitance. In general, the lateral dimensions of the cell are much larger than the cell gap, which allows one to model the behavior in the interior ignoring the influence of fringe fields and the extended nature of the electric field. Thus for a model problem domain, we can take an interior subdomain $\Omega$, as shown in Fig. 1-while this depiction is two dimensional, the actual domain that we envision is three dimensional, hexahedral.

Confining substrates can be treated in various ways in order to coerce (or at least encourage) the neighboring molecules to orient in desired ways. If the control exercised in this fashion is sufficiently effective, then one can assume a "strong anchoring condition" (Dirichlet boundary condition) on the director; otherwise, one will use a "weak anchoring condition" (a natural boundary condition arising from a surface anchoring energy) - this is discussed in Sec. 2.2 below. The boundary tractions and couple stresses that we study here behave differently with respect to strong versus weak boundary conditions, and so to account for this, we incorporate both, imposing the following boundary conditions on $\boldsymbol{n}$ in our model problem: on $\Gamma_{1}$ (strong anchoring, $\boldsymbol{n}=\boldsymbol{n}_{0}$ ), on $\Gamma_{2}$ (weak anchoring, (11b) below), on $\Gamma_{3}$ (periodic) - it is common (though not necessary) for $\boldsymbol{n}_{0}$ to be constant. The electric potential $U$ is constant on $\Gamma_{1}$ and also on $\Gamma_{2}$ and must have a difference of $V$ between them, which is imposed by requiring $U=-V / 2$ on $\Gamma_{1}$ and $U=V / 2$ on $\Gamma_{2}$-the potential is relative to a value of zero at infinity. As is the case with $\boldsymbol{n}$, the potential $U$ satisfies periodic boundary conditions on opposing sides of $\Gamma_{3}$.

While the model problem geometry and boundary conditions are somewhat idealized, they embody several features that are of interest to us, and they allow for several types of solutions that are encountered in practice. These include one-dimensional solutions $(\boldsymbol{n}=$ $\boldsymbol{n}\left(x_{3}\right)$, uniform in $\left.x_{1}, x_{2}\right)$, two-dimensional solutions $\left(\boldsymbol{n}=\boldsymbol{n}\left(x_{1}, x_{3}\right)\right.$, periodic in $x_{1}$, uniform in $x_{2}$, "stripes"), and three-dimensional solutions $\left(\boldsymbol{n}=\boldsymbol{n}\left(x_{1}, x_{2}, x_{3}\right)\right.$, periodic in $x_{1}$, periodic in $x_{2}$, "cells"). Here the coordinates are with respect to a frame with $e_{1}$ and $e_{2}$ in the lateral, in-plane directions, and $e_{3}$ bridging the cell gap. In reality, the periodicities of such solutions would not be known a-priori but would instead be determined in a self-consistent way with the equilibrium fields. We ignore this aspect, which would only add unnecessary complexity to our efforts here.

The theories from which we work are variational in nature. We consider a model for a material in a nematic liquid crystal phase (chiral or achiral) that can be expressed as an 
integral functional of the director field $\boldsymbol{n}$ and the electric potential field $U$ :

$$
\mathscr{F}[\boldsymbol{n}, U]=\int_{\Omega} W(x, \boldsymbol{n}, \nabla \boldsymbol{n}, U, \nabla U) \mathrm{d} V+\int_{\Gamma_{2}} W_{\mathrm{s}}(x, \boldsymbol{n}) \mathrm{d} S .
$$

Here $\Omega$ is the region occupied by the material (as depicted in Fig. 1), $W$ is the free-energy density (per unit volume), $\Gamma_{2}$ is the weak-anchoring portion of the boundary of $\Omega$, and $W_{\mathrm{s}}$ is the surface anchoring energy (per unit area). Stationary points of $\mathscr{F}$ (subject to the unitlength constraint on $\boldsymbol{n}$ and appropriate boundary conditions) determine equilibrium states of the system. The indicated dependencies of $W$ and $W_{\mathrm{s}}$ are sufficiently general to encompass the variety of effects of interest to us, which we now elaborate.

\subsection{Free-energy density}

The free-energy densities in which we are interested may embody all or some of the influences of distortional elasticity, an electric field, flexoelectricity, a prescribed distribution of free electric charge, a magnetic field, and gravity. The relevant relations from electrostatics (macroscopic Maxwell equations, Maxwell-Faraday theory) are

$$
\boldsymbol{D}=\varepsilon_{0} \boldsymbol{E}+\boldsymbol{P}, \quad \operatorname{div} \boldsymbol{D}=\rho_{\mathrm{f}}, \quad \boldsymbol{E}=-\nabla U,
$$

where $\boldsymbol{D}$ is the electric displacement, $\varepsilon_{0}$ the vacuum permittivity, $\boldsymbol{E}$ the electric field, $\boldsymbol{P}$ the polarization, and $\rho_{\mathrm{f}}$ the density of free charge [57, Ch. III]. The individual contributions to $W$ that we envision can be grouped as follows, with dependencies indicated:

$$
W(x, \boldsymbol{n}, \nabla \boldsymbol{n}, U, \nabla U)=W_{\mathrm{e}}(\boldsymbol{n}, \nabla \boldsymbol{n})+W_{\mathrm{E}}(\boldsymbol{n}, \nabla \boldsymbol{n}, \nabla U)+\rho_{\mathrm{f}}(x) U+W_{\mathrm{H}}(\boldsymbol{n})+\rho_{\mathrm{m}} g h(x) .
$$

Here $W_{\mathrm{e}}$ denotes the distortional elasticity of the director field, $W_{\mathrm{E}}$ and $W_{\mathrm{H}}$ stand for the free-energy contributions associated with the electric and magnetic fields, $\rho_{\mathrm{m}}$ is the constant mass density, $g$ is the gravitational constant, and the function $h$ gives the height of the point $x$ above some reference elevation $-g h$ is the gravitational potential per unit mass for an incompressible material.

Through intermolecular forces, the liquid crystal director field is encouraged to assume certain simple arrangements: uniform parallel alignment (in the nematic phase), helical structures (in the cholesteric or chiral nematic phase). Other influences (such as boundary conditions and external fields) can cause $\boldsymbol{n}$ to deviate from these preferred orientations, and the distortional elasticity $W_{\mathrm{e}}$ gives the energetic penalty for this. In macroscopic models, the most commonly used expression for $W_{\mathrm{e}}$ is the Oseen-Frank model and is given by

$$
2 W_{\mathrm{e}}=K_{1}(\operatorname{div} \boldsymbol{n})^{2}+K_{2}\left(\boldsymbol{n} \cdot \operatorname{curl} \boldsymbol{n}+q_{0}\right)^{2}+K_{3}|\boldsymbol{n} \times \operatorname{curl} \boldsymbol{n}|^{2}+K_{24}\left[\operatorname{tr}(\nabla \boldsymbol{n})^{2}-(\operatorname{div} \boldsymbol{n})^{2}\right],
$$

with $K_{1}, K_{2}, K_{3}$, and $K_{24}$ the elastic constants and $q_{0}$ the spontaneous twist parameter (the wave number associated with the cholesteric pitch) [56, Sec. 2.2], [63, Sec. 3.2]. These parameters depend on various factors: the type of material, the temperature, the concentration of chiral dopant, etc. Different symmetries are associated with the presence or absence of $q_{0}$ : the case $q_{0}=0$ corresponds to the "nematic energy," which satisfies

$$
W_{\mathrm{e}}\left(\mathbf{Q} \boldsymbol{n}, \mathbf{Q} \nabla \boldsymbol{n} \mathbf{Q}^{T}\right)=W_{\mathrm{e}}(\boldsymbol{n}, \nabla \boldsymbol{n}), \quad \forall \mathbf{Q} \in O(\mathscr{V}),
$$

while $q_{0} \neq 0$ gives the "cholesteric energy," which satisfies

$$
W_{\mathrm{e}}\left(\mathbf{R} \boldsymbol{n}, \mathbf{R} \nabla \boldsymbol{n} \mathbf{R}^{T}\right)=W_{\mathrm{e}}(\boldsymbol{n}, \nabla \boldsymbol{n}), \quad \forall \mathbf{R} \in S O(\mathscr{V}) .
$$


Here $O(\mathscr{V})$ and $S O(\mathscr{V})$ denote the orthogonal group and the special orthogonal group on the underlying vector space $\mathscr{V}$. We note that while the $S O \mathscr{V})$ invariance holds for the full expression $W_{\mathrm{e}}$ above, it is generally the case that one takes $K_{24}=0$ in the cholesteric energythe reasons for this are given in [56, Sec. 2.2.2] and [63, Sec. 3.2 Remark 2]. The simplest prototype $W_{\mathrm{e}}$ is given by the "equal elastic constants model" ( $q_{0}=0, K_{1}=K_{2}=K_{3}=K_{24}=$ $K)$ :

$$
W_{\mathrm{e}}=\frac{1}{2} K|\nabla \boldsymbol{n}|^{2} .
$$

The precise form of $W_{\mathrm{e}}$ does not matter in our development. All that matters is that $W_{\mathrm{e}}$ depends only on $\boldsymbol{n}$ and $\nabla \boldsymbol{n}$ and that it is properly frame indifferent (i.e., satisfies (3) above). Distortion of the director field can lead to the creation and transmission of forces and torques [24. Secs. 3.1.5, 3.5.2], and one of our main objectives here is to determine expressions for the stress tensor and couple stress tensor related to this in a more general context than has been considered in the past. In the hydrodynamic setting, director distortion can induce fluid motion, and fluid motion can induce reorientation of the director field-here, however, we restrict our attention to statics.

The most common situation for liquid-crystal devices and experiments involving electric fields is for the fields to be produced by electrodes held at constant potential by some voltage source (or battery), for which the contribution to the free energy is usually found written in the form

$$
W_{\mathrm{E}}=-\int_{0}^{\boldsymbol{E}} \boldsymbol{D} \cdot \mathrm{d} \boldsymbol{E} .
$$

See for example [24, Sec. 3.3.1] or [38, Sec. 6.2] or [56, Sec. 2.3]. This expression gives the electrostatic free-energy density at a point expressed in terms of the intensity of the electric field as the field is built up incrementally, reversibly from $\boldsymbol{E}=\mathbf{0}$ to its final value. The equivalent relation is given in differential form in [40, Eqn. (10.9)]. To determine $W_{\mathrm{E}}$ in a particular context, one must prescribe the dependence of $\boldsymbol{D}$ on $\boldsymbol{E}$. Here we assume that the polarization $\boldsymbol{P}$ consists of an induced polarization that is linear in $\boldsymbol{E}$ plus a flexoelectric polarization (which is caused by the distortion of the director field itself):

$$
\boldsymbol{P}=\varepsilon_{0} \chi_{\mathrm{e}} \boldsymbol{E}+\boldsymbol{P}_{\mathrm{f}} \Rightarrow \boldsymbol{D}=\boldsymbol{\varepsilon} \boldsymbol{E}+\boldsymbol{P}_{\mathrm{f}}, \quad \varepsilon=\varepsilon_{0}\left(\mathbf{I}+\chi_{\mathrm{e}}\right) .
$$

Here $\chi_{\mathrm{e}}$ is the electric susceptibility tensor and $\varepsilon$ the dielectric permittivity tensor, which for a uniaxial nematic liquid crystal has the "transversely isotropic" form

$$
\varepsilon=\varepsilon_{0}\left[\varepsilon_{\perp} \mathbf{I}+\Delta \varepsilon(\boldsymbol{n} \otimes \boldsymbol{n})\right], \quad \Delta \varepsilon:=\varepsilon_{\|}-\varepsilon_{\perp},
$$

with $\varepsilon_{\|}$and $\varepsilon_{\perp}$ the relative permittivities parallel to $\boldsymbol{n}$ and perpendicular to $\boldsymbol{n}$. The tensor field $\varepsilon$ is real, symmetric, and positive definite, and the medium is inhomogeneous and anisotropic in general. Linearity of the induced polarization is a valid assumption for the most common situations, though it is acknowledged that it holds more properly in the frequency domain [33, Sec. I.4]. The flexoelectric polarization is taken in the form

$$
\boldsymbol{P}_{\mathrm{f}}=e_{\mathrm{s}}(\operatorname{div} \boldsymbol{n}) \boldsymbol{n}+e_{\mathrm{b}} \boldsymbol{n} \times \operatorname{curl} \boldsymbol{n},
$$

with $e_{\mathrm{s}}$ and $e_{\mathrm{b}}$ the "splay" and "bend" flexoelectric coefficients [38, Sec. 4.1].

With this model for $\boldsymbol{D}(\boldsymbol{E})$, one can compute $W_{\mathrm{E}}$ (most readily by expressing all fields in terms of an orthonormal basis for $\varepsilon$ at the point):

$$
W_{\mathrm{E}}=-\int_{\mathbf{0}}^{\boldsymbol{E}}\left(\varepsilon \boldsymbol{E}+\boldsymbol{P}_{\mathrm{f}}\right) \cdot \mathrm{d} \boldsymbol{E}=-\frac{1}{2} \boldsymbol{\varepsilon} \boldsymbol{E} \cdot \boldsymbol{E}-\boldsymbol{P}_{\mathrm{f}} \cdot \boldsymbol{E}=-\frac{1}{2} \varepsilon \nabla U \cdot \nabla U+\boldsymbol{P}_{\mathrm{f}} \cdot \nabla U .
$$


See for example [46, Sec. II.C]. If flexoelectric terms are ignored (taking $\boldsymbol{P}_{\mathrm{f}}=\mathbf{0}$ ), this simplifies to the more familiar form

$$
W_{\mathrm{E}}=-\frac{1}{2} \boldsymbol{D} \cdot \boldsymbol{E}, \quad \boldsymbol{D}=\boldsymbol{\varepsilon} \boldsymbol{E}
$$

We note that the inclusion of flexoelectric terms formally takes us outside the realm of a "linear dielectric medium" (since $\boldsymbol{D}=\boldsymbol{\varepsilon} \boldsymbol{E}+\boldsymbol{P}_{\mathrm{f}}$ is an affine function of $\boldsymbol{E}$ and not the result of a linear transformation acting on $\boldsymbol{E}$ ). We also note that the presence of $\boldsymbol{P}_{\mathrm{f}}$ creates a coupling between the electric field and $\nabla \boldsymbol{n}$, in addition to the coupling between $\boldsymbol{E}$ and $\boldsymbol{n}$ already present in the simplified expression above.

The potential associated with the magnetic field can be taken in the form

$$
W_{\mathrm{H}}=-\frac{1}{2} \mu_{0} \Delta \chi(\boldsymbol{H} \cdot \boldsymbol{n})^{2}, \quad \Delta \chi:=\chi_{\|}-\chi_{\perp},
$$

with $\mu_{0}$ the free-space permeability, $\chi_{\|}$and $\chi_{\perp}$ the magnetic susceptibilities parallel to $\boldsymbol{n}$ and perpendicular to $\boldsymbol{n}$, and $\boldsymbol{H}$ the magnetic field (which can be assumed to be constant, uniform throughout the material) [56. Sec. 2.3], [63, Sec. 4.1]. In reality, $\boldsymbol{H}$ is affected by the medium, but at a negligible level for typical liquid crystal materials, as we have noted in Sec.11 This can be seen from the relevant formulas of magnetostatics for a uniaxial nematic:

$$
\begin{gathered}
\boldsymbol{B}=\mu_{0}(\boldsymbol{M}+\boldsymbol{H}), \quad \boldsymbol{M}=\chi_{\mathrm{m}} \boldsymbol{H}, \quad \chi_{\mathrm{m}}=\chi_{\perp} \mathbf{I}+\Delta \chi(\boldsymbol{n} \otimes \boldsymbol{n}), \\
W_{\mathrm{H}}=-\frac{1}{2} \boldsymbol{B} \cdot \boldsymbol{H}=-\frac{1}{2} \mu_{0}\left[\left(1+\chi_{\perp}\right) H^{2}+\Delta \chi(\boldsymbol{H} \cdot \boldsymbol{n})^{2}\right] .
\end{gathered}
$$

Here $\boldsymbol{B}$ is the magnetic induction, $\boldsymbol{M}$ the magnetization, and $\chi_{\mathrm{m}}$ the magnetic susceptibility tensor [57, Ch. IV]. In terms of the scalar magnetic potential $U_{\mathrm{m}}$ (with $\boldsymbol{H}=-\nabla U_{\mathrm{m}}$ ), the Maxwell equation for the magnetic induction $(\operatorname{div} \boldsymbol{B}=0)$ takes the form

$$
\Delta U_{\mathrm{m}}+\frac{\Delta \chi}{1+\chi_{\perp}} \operatorname{div}\left[\left(\nabla U_{\mathrm{m}} \cdot \boldsymbol{n}\right) \boldsymbol{n}\right]=0,
$$

and the term involving $\boldsymbol{n}$ is negligible (at our level of modeling) by virtue of the fact that $\Delta \chi /\left(1+\chi_{\perp}\right)=O\left(10^{-6}\right)$ for typical liquid crystals-see [24. Sec. 3.2.1] or [56, Appendix D]. The analogous equation for the electric potential $U$ (from $\operatorname{div} \boldsymbol{D}=\rho_{\mathrm{f}}$ ) is

$$
\Delta U+\frac{\Delta \varepsilon}{\varepsilon_{\perp}} \operatorname{div}[(\nabla U \cdot \boldsymbol{n}) \boldsymbol{n}]=\frac{1}{\varepsilon_{0} \varepsilon_{\perp}}\left(\operatorname{div} \boldsymbol{P}_{\mathrm{f}}-\rho_{\mathrm{f}}\right),
$$

and the coupling term satisfies $\Delta \varepsilon / \varepsilon_{\perp}=O(1)$-again see [56, Appendix D]. For this reason, the influence of the medium on the electric field cannot be neglected-the electric field influences the director field and vice versa, and the two fields must be determined in a coupled, self-consistent way (cf. [56, Sec. 3.5]). The term $-\frac{1}{2} \mu_{0}\left(1+\chi_{\perp}\right) H^{2}$ in the expression for $-\frac{1}{2} \boldsymbol{B} \cdot \boldsymbol{H}$ above would just add a constant to $W$ and is generally discarded. We note that while $\nabla \boldsymbol{H}$ is negligibly small in typical liquid crystal settings (the justification for discarding the term $-\frac{1}{2} \mu_{0}\left(1+\chi_{\perp}\right) H^{2}$ ), if circumstances were such that it needed to be taken into account, then it would contribute to the body force-see [55, Sec. 3.1.4] or [56, Sec. 2.4.2].

The term $\rho_{\mathrm{f}} U$ is not common but is included here for the purpose of illustration. For the systems we are interested in modeling, the only free-charge distributions typically encountered are very small concentrations of ionic impurities, which are usually dealt with in one of two ways. If DC electric fields are employed, then the mobile ions will cluster into very thin screening layers adjacent to the electrodes of opposite polarity. This can be modeled 
using Poisson-Boltzmann theory, though simpler approximations are usually employedsee [3, Ch. 7] or [34, Sec. 8.5]. As an alternative, one could use AC fields at a frequency such that the force on the ions $\left(\rho_{\mathrm{f}} \boldsymbol{E}\right)$ effectively time-averages to zero, while the director (which couples to $E^{2}$ ) effectively responds to the field associated with the RMS voltage. In a similar vein, the gravitational potential $\rho_{\mathrm{m}} g h$ is not usually encountered but is included for illustration. All of the other terms discussed above are common. The simplest prototype of (2) that embodies liquid-crystal distortional elasticity and a coupled electric field is

$$
W(\boldsymbol{n}, \nabla \boldsymbol{n}, \nabla U)=\frac{1}{2} K|\nabla \boldsymbol{n}|^{2}-\frac{1}{2} \varepsilon(\boldsymbol{n}) \nabla U \cdot \nabla U,
$$

with the dielectric tensor $\varepsilon$ having the form (5). In most of what follows, we simply work with $W$ in the generic form $W(x, \boldsymbol{n}, \nabla \boldsymbol{n}, U, \nabla U)$.

\subsection{Surface anchoring energy}

The surface anchoring energy $W_{\mathrm{s}}$ can take a variety of forms, as there are a number of different techniques used to try to control the orientational properties of a liquid-crystal material at an interface to a substrate-see for example [3, 28, 52,53,54]. A reasonable model is to assume that $W_{\mathrm{s}}$ depends on the director, the normal to the surface, and a distinguished direction tangent to the surface at the point. Thus we assume

$$
W_{\mathrm{s}}=W_{\mathrm{s}}(x, \boldsymbol{n} ; \boldsymbol{\nu}, \boldsymbol{\tau}),
$$

where $\nu$ is the unit normal to the surface at the point $x$ and $\tau$ is a unit-vector tangent to the surface at that point. We note that in circumstances in which curvature effects of the anchoring surface are significant, that would warrant the inclusion of additional terms in $W_{\mathrm{s}}$ involving surface gradients of $\boldsymbol{n}$ and/or $\boldsymbol{\nu}$ and/or $\boldsymbol{\tau}$-see [21] and references contained therein.

The anchoring potential should conform to the same symmetries as the free-energy density: evenness,

$$
W_{\mathrm{s}}(x,-\boldsymbol{n} ; \boldsymbol{\nu}, \boldsymbol{\tau})=W_{\mathrm{s}}(x, \boldsymbol{n} ; \boldsymbol{\nu}, \boldsymbol{\tau}),
$$

and either invariance with respect to the full orthogonal group (case of "nematic symmetry"),

$$
W_{\mathrm{s}}(x, \mathbf{Q} \boldsymbol{n} ; \mathbf{Q} \boldsymbol{\nu}, \mathbf{Q} \boldsymbol{\tau})=W_{\mathrm{s}}(x, \boldsymbol{n} ; \boldsymbol{\nu}, \boldsymbol{\tau}), \quad \forall \mathbf{Q} \in O(\mathscr{V}),
$$

or invariance with respect to only proper orthogonal transformations (case of "cholesteric symmetry"),

$$
W_{\mathrm{s}}(x, \mathbf{R} \boldsymbol{n} ; \mathbf{R} \boldsymbol{\nu}, \mathbf{R} \boldsymbol{\tau})=W_{\mathrm{s}}(x, \boldsymbol{n} ; \boldsymbol{\nu}, \boldsymbol{\tau}), \quad \forall \mathbf{R} \in S O(\mathscr{V}) .
$$

Representation theory tells us that in the former case, $W_{\mathrm{s}}$ must be of the form

$$
W_{\mathrm{s}}(x, \boldsymbol{n} ; \boldsymbol{\nu}, \boldsymbol{\tau})=\widetilde{W}_{\mathrm{s}}(x, \boldsymbol{n} \cdot \boldsymbol{\nu}, \boldsymbol{n} \cdot \boldsymbol{\tau}),
$$

for some function $\widetilde{W}_{\mathrm{s}}$ that satisfies

$$
\widetilde{W}_{\mathrm{s}}\left(\arg _{1},-\arg _{2},-\arg _{3}\right)=\widetilde{W}_{\mathrm{s}}\left(\arg _{1}, \arg _{2}, \arg _{3}\right),
$$

while in the latter case, we must have

$$
\begin{gathered}
W_{\mathrm{s}}(x, \boldsymbol{n} ; \boldsymbol{\nu}, \boldsymbol{\tau})=\widetilde{W}_{\mathrm{s}}\left(x, \boldsymbol{n} \cdot \boldsymbol{\nu}, \boldsymbol{n} \cdot \boldsymbol{\tau}, \boldsymbol{n} \cdot \boldsymbol{\tau}_{2}\right), \quad \boldsymbol{\tau}_{2}:=\boldsymbol{\nu} \times \boldsymbol{\tau} \\
\widetilde{W}_{\mathrm{s}}\left(\arg _{1},-\arg _{2},-\arg _{3},-\arg _{4}\right)=\widetilde{W}_{\mathrm{s}}\left(\arg _{1}, \arg _{2}, \arg _{3}, \arg _{4}\right) .
\end{gathered}
$$


See [61. Sec. 11].

A simple example of such an energy (which obeys the full nematic symmetry) is

$$
\begin{aligned}
W_{\mathrm{s}} & =-\frac{1}{2} W_{0}\left(\boldsymbol{n} \cdot \boldsymbol{n}_{0}\right)^{2}, \quad \boldsymbol{n}_{0}=a \boldsymbol{\nu}+b \boldsymbol{\tau}, \quad a^{2}+b^{2}=1 \\
& =-\frac{1}{2} W_{0}\left[a^{2}(\boldsymbol{n} \cdot \boldsymbol{\nu})^{2}+2 a b(\boldsymbol{n} \cdot \boldsymbol{\nu})(\boldsymbol{n} \cdot \boldsymbol{\tau})+b^{2}(\boldsymbol{n} \cdot \boldsymbol{\tau})^{2}\right] .
\end{aligned}
$$

Here the positive constant $W_{0}$ is the "anchoring strength" and $\boldsymbol{n}_{0}$ the "easy axis." The potential simply encourages the director to align with $\pm \boldsymbol{n}_{0}$ and penalizes it if it doesn't. Among other possibilities, such an energy allows one to model a weak "pre-tilt" boundary condition, in which the preferred orientation $\boldsymbol{n}_{0}$ is tilted some amount in a certain direction from the normal. The $x$ dependence that we envision is through the anchoring coefficients. While parameters such as $W_{0}$ above are almost always taken to be constants, it is conceivable that if the anchoring strength were known to vary appreciably across the surface, then one may want to model $W_{0}=W_{0}(x)$.

For the situation in which the in-plane vector $\tau$ is associated with the "rubbing direction" of grooves rubbed into a polymer alignment layer, the anchoring energy should be indifferent to $\tau$ versus $-\tau$, as discussed in [28]. In that case, we would require the additional symmetry

$$
W_{\mathrm{s}}(x, \boldsymbol{n} ; \boldsymbol{\nu},-\boldsymbol{\tau})=W_{\mathrm{s}}(x, \boldsymbol{n} ; \boldsymbol{\nu}, \boldsymbol{\tau}),
$$

which would necessitate that the nematic function satisfy both

$$
\begin{aligned}
& \widetilde{W}_{\mathrm{s}}\left(\arg _{1},-\arg _{2}, \arg _{3}\right)=\widetilde{W}_{\mathrm{s}}\left(\arg _{1}, \arg _{2}, \arg _{3}\right) \\
& \widetilde{W}_{\mathrm{s}}\left(\arg _{1}, \arg _{2},-\arg _{3}\right)=\widetilde{W}_{\mathrm{s}}\left(\arg _{1}, \arg _{2}, \arg _{3}\right)
\end{aligned}
$$

and that the cholesteric function obey

$$
\begin{gathered}
\widetilde{W}_{\mathrm{s}}\left(\arg _{1},-\arg _{2}, \arg _{3}, \arg _{4}\right)=\widetilde{W}_{\mathrm{s}}\left(\arg _{1}, \arg _{2}, \arg _{3}, \arg _{4}\right) \\
\widetilde{W}_{\mathrm{s}}\left(\arg _{1}, \arg _{2},-\arg _{3},-\arg _{4}\right)=\widetilde{W}_{\mathrm{s}}\left(\arg _{1}, \arg _{2}, \arg _{3}, \arg _{4}\right) .
\end{gathered}
$$

The difference between the types of anchoring energies allowed in the case of nematic symmetry versus cholesteric symmetry (for the models invariant with respect to $\pm \boldsymbol{\tau}$ ) can be illustrated by example. Consider the case of weak homeotropic anchoring, with easy axis $\boldsymbol{n}_{0}=\boldsymbol{\nu}$. For the common situation of polynomial expressions of at most quadratic order, the set of admissible terms comprises

$$
(\boldsymbol{n} \cdot \boldsymbol{\nu})^{2},(\boldsymbol{n} \cdot \boldsymbol{\tau})^{2},\left(\boldsymbol{n} \cdot \boldsymbol{\tau}_{2}\right)^{2},(\boldsymbol{n} \cdot \boldsymbol{\tau})\left(\boldsymbol{n} \cdot \boldsymbol{\tau}_{2}\right),
$$

with only two of the first three terms being independent, by virtue of the relation

$$
(\boldsymbol{n} \cdot \boldsymbol{\nu})^{2}+(\boldsymbol{n} \cdot \boldsymbol{\tau})^{2}+\left(\boldsymbol{n} \cdot \boldsymbol{\tau}_{2}\right)^{2}=1 .
$$

The first three admissible terms satisfy the nematic symmetry assumptions (since $\left(\boldsymbol{n} \cdot \boldsymbol{\tau}_{2}\right)^{2}=$ $\left.1-(\boldsymbol{n} \cdot \boldsymbol{\nu})^{2}-(\boldsymbol{n} \cdot \boldsymbol{\tau})^{2}\right)$, while the fourth term does not. Thus one can construct nematic anchoring energies of the form

$$
W_{\mathrm{s}}=-\frac{1}{2} W_{0}(\boldsymbol{n} \cdot \boldsymbol{\nu})^{2} \quad \text { or } \quad W_{\mathrm{s}}=\frac{1}{2} W_{1}(\boldsymbol{n} \cdot \boldsymbol{\tau})^{2}+\frac{1}{2} W_{2}\left(\boldsymbol{n} \cdot \boldsymbol{\tau}_{2}\right)^{2} .
$$

With $W_{0}, W_{1}$, and $W_{2}$ all positive, both energies attain their minimum values at $\boldsymbol{n}= \pm \boldsymbol{\nu}$. The level sets of the first energy (projected onto the tangent plane to the surface at the point) 
are circles, while those of the second are ellipses with axes aligned with $\tau$ and $\tau_{2}$. Such potentials are said to be of "Rapini-Papoular type" (see for example [3, Sec. 2.2]). A slight generalization of this model, which obeys the cholesteric symmetry but not the nematic symmetry, is

$$
W_{\mathrm{s}}=\frac{1}{2} W_{1}(\boldsymbol{n} \cdot \boldsymbol{\tau})^{2}+\frac{1}{2} W_{2}\left(\boldsymbol{n} \cdot \boldsymbol{\tau}_{2}\right)^{2}+W_{3}(\boldsymbol{n} \cdot \boldsymbol{\tau})\left(\boldsymbol{n} \cdot \boldsymbol{\tau}_{2}\right) .
$$

With $W_{1}, W_{2}>0$ and $W_{3}^{2}<W_{1} W_{2}$, this potential still has its minimum at $\boldsymbol{n}= \pm \boldsymbol{\nu}$, but the $W_{3}$ term penalizes the twist differently in different directions (clockwise versus counterclockwise) and causes the axes of the elliptical level sets to be rotated relative to $\tau$ and $\tau_{2}$. More aspects of the physical significance of such a model are discussed in [28].

The analogous case of weak planar anchoring can be expressed

$$
W_{\mathrm{s}}=-\frac{1}{2} W_{1}(\boldsymbol{n} \cdot \boldsymbol{\tau})^{2}-\frac{1}{2} W_{2}\left(\boldsymbol{n} \cdot \boldsymbol{\tau}_{2}\right)^{2}+W_{3}(\boldsymbol{n} \cdot \boldsymbol{\tau})\left(\boldsymbol{n} \cdot \boldsymbol{\tau}_{2}\right), \quad W_{1}, W_{2} \geq 0, \quad W_{3}^{2} \leq W_{1} W_{2},
$$

with $W_{1}$ and $W_{2}$ not both zero. The easy axis would be determined by the weights: $W_{1}>W_{2}$ and $W_{3}=0 \Rightarrow \boldsymbol{n}_{\min }= \pm \boldsymbol{\tau}, W_{2}>W_{1}$ and $W_{3}=0 \Rightarrow \boldsymbol{n}_{\min }= \pm \boldsymbol{\tau}_{2}$. With $W_{3} \neq 0$, the easy axis would lie in the plane $\operatorname{span}\left\{\tau, \tau_{2}\right\}$ but would not be aligned with either of those directions.

The various representations for $W_{\mathrm{s}}$ have consequences concerning quantities such as $\partial W_{\mathrm{s}} / \partial \boldsymbol{n}, \partial W_{\mathrm{s}} / \partial \boldsymbol{\nu}$, and $\partial W_{\mathrm{s}} / \partial \tau$, but we shall not require them in what follows and therefore omit those details. In our development, we can assume the more general cholesteric symmetry (with or without the assumption of invariance with respect to $\pm \boldsymbol{\tau}$ ), viewing the corresponding nematic energy as a special case. For the most part, we simply work with the generic expression $W_{\mathrm{s}}(x, \boldsymbol{n} ; \boldsymbol{\nu}, \boldsymbol{\tau})$, which is capable of modeling most of the cases of interest.

\section{Orientational Equilibrium and the Issue of Variational Compatibility}

The issue of variational compatibility concerns the following. The Euler-Lagrange equations associated with the functional (1), when combined with appropriate boundary conditions and the constraint $|\boldsymbol{n}|=1$, completely determine the equilibrium director field $\boldsymbol{n}$ and electric potential field $U$. These solutions, however, must also satisfy the static limit of the EricksenLeslie equations for liquid crystal flows. In this section, we frame this question and present Ericksen's suggested approach to resolving it.

\subsection{Euler-Lagrange equations}

The equilibrium Euler-Lagrange equations associated with a functional of the form (1) follow from the variations $\delta_{\boldsymbol{n}} \mathscr{F}=0$ (subject to $|\boldsymbol{n}|=1$ ) and $\delta_{U} \mathscr{F}=0$, from which one obtains

$$
\begin{gathered}
\operatorname{div}\left(\frac{\partial W}{\partial \nabla \boldsymbol{n}}\right)-\frac{\partial W}{\partial \boldsymbol{n}}+\lambda \boldsymbol{n}=\mathbf{0}, \quad \operatorname{div}\left(\frac{\partial W}{\partial \nabla U}\right)-\frac{\partial W}{\partial U}=0, \quad \text { in } \Omega \\
\left(\frac{\partial W}{\partial \nabla \boldsymbol{n}}\right) \boldsymbol{\nu}-\frac{\partial W_{\mathrm{s}}}{\partial \boldsymbol{n}}+\mu \boldsymbol{n}=\mathbf{0}, \quad \text { on } \Gamma_{2} .
\end{gathered}
$$


Here $11 \mathrm{~b}$ ) is the natural boundary condition associated with the weak anchoring potential, and $\lambda$ and $\mu$ are Lagrange-multiplier fields associated with the pointwise unit-length constraint on $\boldsymbol{n}$. Equation (11a) 2 can be seen to yield the appropriate electrostatics equation: using (2) and (7),

$$
\frac{\partial W}{\partial \nabla U}=-\varepsilon \nabla U+\boldsymbol{P}_{\mathrm{f}}=\boldsymbol{\varepsilon} \boldsymbol{E}+\boldsymbol{P}_{\mathrm{f}}=\boldsymbol{D}, \quad \frac{\partial W}{\partial U}=\rho_{\mathrm{f}},
$$

from which follows

$$
\operatorname{div}\left(\frac{\partial W}{\partial \nabla U}\right)-\frac{\partial W}{\partial U}=0 \Rightarrow \operatorname{div} \boldsymbol{D}=\rho_{\mathrm{f}}
$$

We note that in the thermodynamic formalism, $\boldsymbol{D}$ and $-\boldsymbol{E}$ are conjugate variables [40, Sec. 10]; so the relationship $\partial W / \partial \nabla U=-\partial W / \partial \boldsymbol{E}=\boldsymbol{D}$ is expected (and in fact is built into (4)).

The Euler-Lagrange equations $(11 \mathrm{a})$ plus $(11 \mathrm{~b})$ and the essential boundary conditions completely determine equilibrium director fields $\boldsymbol{n}$ and electric potential fields $U$ in a coupled, self-consistent way. The problem is nonlinear, however, and so it is possible for there to be more than one distinct solution pair for a given set of parameters. In this case, any solution pair of least free energy provides a globally stable state of the system. The issue of "variational compatibility" concerns the consistency of these solutions with the static limit of the Ericksen-Leslie equations, which form the hydrodynamic theory of liquid crystal flows at this modeling scale.

\subsection{Static limit of Ericksen-Leslie hydrodynamics}

The hydrostatic limit of the Ericksen-Leslie equations is discussed in [24, Sec. 3.5], [55, Sec. 3.1], and [56, Sec. 2.4]. In this limit, the hydrodynamic system reduces to a balance of forces (associated with conservation of linear momentum) and a balance of torques (associated with conservation of angular momentum). In the classical setting, these limiting equations are usually written

$$
\begin{gathered}
\operatorname{div} \mathbf{T}+\boldsymbol{F}=\mathbf{0} \\
\operatorname{div}\left(\frac{\partial W_{\mathrm{e}}}{\partial \nabla \boldsymbol{n}}\right)-\frac{\partial W_{\mathrm{e}}}{\partial \boldsymbol{n}}+\boldsymbol{G}+\lambda \boldsymbol{n}=\mathbf{0}
\end{gathered}
$$

Here $\mathbf{T}$ is the Cauchy stress tensor (associated with contact forces)

$$
\mathbf{T}=-p \mathbf{I}-(\nabla \boldsymbol{n})^{T} \frac{\partial W_{\mathrm{e}}}{\partial \nabla \boldsymbol{n}}
$$

$\boldsymbol{F}$ is the external body force (force per unit volume), $\boldsymbol{G}$ is the external generalized force acting on the director (force per unit area) - the torque density (torque per unit volume) is $\boldsymbol{n} \times \boldsymbol{G}$-the Lagrange multiplier field $\lambda$ is associated with the pointwise constraint $|\boldsymbol{n}|=$ 1 , and $p$ is the pressure field associated with incompressibility. The fields $\boldsymbol{F}$ and $\boldsymbol{G}$ are prescribed and depend on the particular system being studied. One can readily interpret (12a) as a force balance. The actual torque balance takes a form different from $(12 \mathrm{~b}$; we shall encounter it later in our development. To show that $12 \mathrm{~b}$ is equivalent to the torque balance requires some effort but is well documented-see the reference cited above. 
For a free-energy density $W$ of the form (2), the director equilibrium equation $(11 \mathrm{a})_{1}$ is consistent with $(12 \mathrm{~b})$ if we identify $\boldsymbol{G}$ as

$$
\boldsymbol{G}=\operatorname{div}\left[\frac{\partial\left(W-W_{\mathrm{e}}\right)}{\partial \nabla \boldsymbol{n}}\right]-\frac{\partial\left(W-W_{\mathrm{e}}\right)}{\partial \boldsymbol{n}}=\operatorname{div}\left(\frac{\partial W_{\mathrm{E}}}{\partial \nabla \boldsymbol{n}}\right)-\frac{\partial W_{\mathrm{E}}}{\partial \boldsymbol{n}}-\frac{\partial W_{\mathrm{H}}}{\partial \boldsymbol{n}} .
$$

In the absence of flexoelectric terms, we would have $\partial W_{\mathrm{E}} / \partial \nabla \boldsymbol{n}=\mathbf{0}$ and a reduced expression for $\partial W_{\mathrm{E}} / \partial \boldsymbol{n}$, and $\boldsymbol{G}$ would take the familiar form

$$
\boldsymbol{G}=\varepsilon_{0} \Delta \varepsilon(\boldsymbol{E} \cdot \boldsymbol{n}) \boldsymbol{E}+\mu_{0} \Delta \chi(\boldsymbol{H} \cdot \boldsymbol{n}) \boldsymbol{H}
$$

with $\boldsymbol{n} \times \boldsymbol{G}$ comprising the dielectric and magnetic torques. The full expression for $\boldsymbol{G}$ (with flexoelectric terms included) is less intuitive. The flexoelectric contributions are complicated: distortion of the director field $\boldsymbol{n}$ induces the flexoelectric polarization $\boldsymbol{P}_{\mathrm{f}}$, as given in (6), which the system strives to align with $\boldsymbol{E}$ in order to minimize the term $-\boldsymbol{P}_{\mathrm{f}} \cdot \boldsymbol{E}$ in $W$. It is also the case that an imposed electric field can induce distortion of $\boldsymbol{n}$ so as to minimize $-\boldsymbol{P}_{\mathrm{f}} \cdot \boldsymbol{E}$ as well (the so-called "inverse flexoelectric effect"). In the literature, a fair amount of attention has been given to various aspects of flexoelectricity in nematics. Some discussion and references can be found in [24, Sec. 3.3.2], [34, Sec. 8.7], and [38, Ch. 4]. Most of these issues are not important for our development.

It is not clear that with $G$ as in (13) with flexoelectric terms included that $112 \mathrm{~b}$ would still imply an appropriate balance of torques. An argument would be required, as the system now contains terms and couplings that were not present in the original analysis relating (12b) to torque balance. We shall see later in our development that working from a free energy that contains the potentials for all fields of external origin leads automatically to the satisfaction of the appropriate force and torque balances, thereby guaranteeing variational compatibility. This is one of the attractive features of our approach.

\subsection{Ericksen's compatibility potential}

It is conceivable that for certain choices of $\boldsymbol{F}$ in $(12 \mathrm{a})$ and $\boldsymbol{G}$ in $(12 \mathrm{~b})$, the variational equilibrium fields do not provide a consistent system for the determination of the pressure field $p$. This is referred to as the issue of "variational compatibility." It was addressed by Ericksen [14] and is recounted in [55. Sec. 3.1.4] and [56, Sec. 2.4]. A sufficient condition for variational compatibility is that there exist a function $\Psi(x, n)$ (the "compatibility potential") that gives rise to both $\boldsymbol{F}$ and $\boldsymbol{G}$ via

$$
\boldsymbol{F}=\frac{\partial \Psi}{\partial x}, \quad \boldsymbol{G}=\frac{\partial \Psi}{\partial \boldsymbol{n}}
$$

Here the partial derivatives are meant to be computed with $x$ and $\boldsymbol{n}$ treated as separate independent variables-the $x$ dependence of $\boldsymbol{n}$ is ignored in computing $\partial \Psi / \partial x$. If such a potential $\Psi$ exists, then it can be shown that consistency of $(12 \mathrm{a})$ and $(12 \mathrm{~b})$ is assured with $p=p_{0}-W_{\mathrm{e}}+\Psi, p_{0}=$ const (see [14] or [55, Sec. 3.1.4] or [56, Sec. 2.4]). If one simply incorporates the potential $\Psi$ into the free-energy density,

$$
W(x, \boldsymbol{n}, \nabla \boldsymbol{n})=W_{\mathrm{e}}(\boldsymbol{n}, \nabla \boldsymbol{n})-\Psi(x, \boldsymbol{n}),
$$

then the balances [12a and (12b) result naturally, as suggested by Ericksen himself later [15, Sec. II.A.1]. 
In [55, Sec. 3.1.4] and [56, Sec. 2.4], it is shown that the case of a uniform external magnetic field fits comfortably into this framework, using

$$
\Psi=\frac{1}{2} \mu_{0} \Delta \chi(\boldsymbol{H} \cdot \boldsymbol{n})^{2}
$$

as we have already used in (2). A gravitational body force fits comfortably into Ericksen's framework as well [56. Sec. 4.2.4], with a compatibility potential

$$
\Psi=-\rho_{\mathrm{m}} g h(x),
$$

as we have also used. The case of an external electric field is more complicated and does not fit as naturally into Ericksen's framework. The electric field is coupled to the director field (and to its gradient, if flexoelectric terms are included) and enters the free energy density as a dependent variable through $U$ and $\nabla U$. At least that is the case in the approach taken herea possible alternate approach would be to treat electrostatics as a PDE constraint. While a magnetic field and a gravitational field can be regarded as "external" fields, an electric field cannot. We now describe the extension of these ideas that serves to handle the general case, in essence following the suggestion of Ericksen, though in a more general context.

\section{Virtual Work Principle}

To determine the quantities of interest to us (body forces and couples, stress tensors, boundary tractions), we follow the same path as originally followed by Ericksen [13] (as recounted in [56, Sec. 2.4.2] and [61, Secs. 127-8]). Before involving boundary conditions and developing general expressions for variations, we determine the forms of the balance of forces and balance of torques that pertain to our system.

4.1 Force balance and torque balance

The free energy of an arbitrary subregion $V \subset \operatorname{int}(\Omega)$ is given by

$$
\mathscr{F}[\boldsymbol{n}, U ; V]=\int_{V} W(x, \boldsymbol{n}, \nabla \boldsymbol{n}, U, \nabla U) \mathrm{d} V,
$$

with $W$ as in 22. We consider small displacements of the form

$$
\begin{gathered}
x^{*}=\Phi_{\varepsilon}(x)=x+\varepsilon \varphi(x)+o(\varepsilon) \\
\boldsymbol{n}^{*}\left(x^{*}\right)=\boldsymbol{n}(x)+\varepsilon \boldsymbol{\psi}(x)+o(\varepsilon) \\
U^{*}\left(x^{*}\right)=U(x)+\varepsilon \Psi(x)+o(\varepsilon) .
\end{gathered}
$$

Here we follow the notation of [23, Sec. 37]. The perturbations $\varphi, \psi$, and $\Psi$ can depend on $\boldsymbol{n}, \nabla \boldsymbol{n}, U$, and $\nabla U$, though we do not express this. In the notation of [13], we would have

$$
\delta x=\varepsilon \varphi, \quad \Delta n=\varepsilon \psi, \quad \Delta U=\varepsilon \Psi .
$$

The change in $\mathscr{F}$ caused by such a displacement is

$$
\Delta \mathscr{F}=\mathscr{F}\left[\boldsymbol{n}^{*}, U^{*} ; V^{*}\right]-\mathscr{F}[\boldsymbol{n}, U ; V], \quad V^{*}=\Phi_{\varepsilon}(V),
$$


with the variation $\delta \mathscr{F}$ the first-order part

$$
\delta \mathscr{F}=\left.\varepsilon \frac{d}{d \varepsilon} \mathscr{F}\left[\boldsymbol{n}^{*}, U^{*} ; V^{*}\right]\right|_{\varepsilon=0},
$$

which is a linear form in $\varphi, \psi$, and $\Psi$.

The state of the system in $V$ is also affected by influences from outside of $V$, which can include forces and fluxes of various types. In equilibrium, all influences must be in balance, in some appropriate sense. The Principle of Virtual Work employed in [13] and utilized here embodies this by expressing the characterization that $(\boldsymbol{n}, U)$ is an equilibrium pair if and only if

$$
\boldsymbol{\delta} \mathscr{F}=\varepsilon \int_{S}(\boldsymbol{t} \cdot \boldsymbol{\varphi}+\boldsymbol{s} \cdot \boldsymbol{\psi}+r \Psi) \mathrm{d} S
$$

for all virtual displacements consistent with the constraints of incompressibility and $|\boldsymbol{n}|=1$. Here $S$ is the boundary of $V$, and $t, s$, and $r$ represent generalized force densities, which are to be identified. The physical dimensions of $t, s$, and $r$ are force per unit area, couple per unit area, and charge per unit area, which leads one to anticipate the forms they will take. Such a principle would ordinarily also involve work associated with volume densities, supported in $V$, in addition to the densities supported on $S$. These are not present in our principle because all such influences have been built into $W$. For (16) to correspond to a virtual displacement "consistent with constraints" means that it must conform to the constraints to first order. Incompressibility demands that displacements preserve volume pointwise (be isochoric), which implies $\operatorname{div} \boldsymbol{\varphi}=0$; while satisfaction of $|\boldsymbol{n}|=1$ to first order requires $\boldsymbol{n} \cdot \boldsymbol{\psi}=0$ at each point.

The expression (17) can be contrasted with that originally employed by Ericksen, which in our notation would take the form

$$
\delta \int_{V} W_{\mathrm{e}} \mathrm{d} V=\varepsilon \int_{V}(\boldsymbol{F} \cdot \boldsymbol{\varphi}+\boldsymbol{G} \cdot \boldsymbol{\psi}) \mathrm{d} V+\varepsilon \int_{S}(\boldsymbol{t} \cdot \boldsymbol{\varphi}+\boldsymbol{s} \cdot \boldsymbol{\psi}) \mathrm{d} S .
$$

The main differences are that the work function above involves only the distortional elasticity of the director field $W_{\mathrm{e}}$ and that the densities $\boldsymbol{F}$ and $\boldsymbol{G}$ are prescribed in the first integral on the right-hand side-(17) corresponds to [14. Eqn. (3.14)], in the more general context of a coupled electric potential field. The expression above leads to the classical balance of forces and balance of torques by following the same path we shall follow below.

Expressions of force balance and torque balance can be deduced from (17) by considering infinitesimal rigid displacements, as done in [41, Sec. II.A], [42, Sec. 2], [55, Sec. 3.1.4], and [56. Sec. 2.4, Remark (i)]. We shall write a general rigid displacement in the form

$$
x^{*}=o+\mathbf{R}\left(x-o^{*}\right), \quad \mathbf{R} \in S O(\mathscr{V}) .
$$

In this notation, the coordinates of the point $x^{*}$ with respect to the frame $\left(o, e_{i}\right)$ are the same as the coordinates of $x$ with respect to $\left(o^{*}, \boldsymbol{e}_{i}^{*}\right)$, with $\boldsymbol{e}_{i}^{*}=\mathbf{R}^{T} \boldsymbol{e}_{i}$. The transformed fields

$$
U^{*}\left(x^{*}\right)=U(x), \quad \boldsymbol{n}^{*}\left(x^{*}\right)=\mathbf{R} \boldsymbol{n}(x)
$$

satisfy

$$
\nabla^{*} U^{*}\left(x^{*}\right)=\mathbf{R} \nabla U(x), \quad \nabla^{*} \boldsymbol{n}^{*}\left(x^{*}\right)=\mathbf{R} \nabla \boldsymbol{n}(x) \mathbf{R}^{T} .
$$

Such transformations are isochoric and preserve $|\boldsymbol{n}|=1$. Special cases include rigid translations $(\mathbf{R}=\mathbf{I})$ and rigid rotations $\left(o^{*}=o\right)$. 
The variation in $\mathscr{F}$ associated with such transformations can be determined by expansion of

$$
\begin{aligned}
\Delta \mathscr{F} & =\mathscr{F}\left[\boldsymbol{n}^{*}, U^{*} ; V^{*}\right]-\mathscr{F}[\boldsymbol{n}, U ; V] \\
& =\int_{V^{*}} W\left(x^{*}, \boldsymbol{n}^{*}, \nabla^{*} \boldsymbol{n}^{*}, U^{*}, \nabla^{*} U^{*}\right) \mathrm{d} V^{*}-\int_{V} W(x, \boldsymbol{n}, \nabla \boldsymbol{n}, U, \nabla U) \mathrm{d} V \\
& =\int_{V}\left[W\left(x^{*}(x), \mathbf{R} \boldsymbol{n}, \mathbf{R} \nabla \boldsymbol{n} \mathbf{R}^{T}, U, \mathbf{R} \nabla U\right)-W(x, \boldsymbol{n}, \nabla \boldsymbol{n}, U, \nabla U)\right] \mathrm{d} V .
\end{aligned}
$$

Here we have used the fact that $\mathrm{d} V^{*}=\mathrm{d} V$ for an isochoric displacement-the volume dilation factor is one everywhere. The parts of $W$ associated with $W_{\mathrm{e}}$ and $W_{\mathrm{E}}$ do not contribute to this expression, since

$$
W_{\mathrm{e}}\left(\mathbf{R} \boldsymbol{n}, \mathbf{R} \nabla \boldsymbol{n} \mathbf{R}^{T}\right)=W_{\mathrm{e}}(\boldsymbol{n}, \nabla \boldsymbol{n}), \quad W_{\mathrm{E}}\left(\mathbf{R} \boldsymbol{n}, \mathbf{R} \nabla \boldsymbol{n} \mathbf{R}^{T}, \mathbf{R} \nabla U\right)=W_{\mathrm{E}}(\boldsymbol{n}, \nabla \boldsymbol{n}, \nabla U),
$$

$\forall \mathbf{R} \in S O(\mathscr{V})$, as can be verified directly. Contributions to $\Delta \mathscr{F}$ can come only from $W_{\mathrm{H}}$ (by virtue of the factor $\left.(\boldsymbol{H} \cdot \boldsymbol{n})^{2}\right)$ and from the terms with explicit $x$ dependence $\left(\rho_{\mathrm{f}}(x) U\right.$ and $\rho_{\mathrm{m}} g h(x)$ ).

For a rigid translation of the form

$$
x^{*}=x+\varepsilon \varphi_{0}, \quad \boldsymbol{n}^{*}\left(x^{*}\right)=\boldsymbol{n}(x), \quad U^{*}\left(x^{*}\right)=U(x),
$$

which corresponds to

$$
\mathbf{R}=\mathbf{I}, \quad o-o^{*}=\varepsilon \varphi_{0}, \quad \varphi=\varphi_{0}, \quad \psi=\mathbf{0}, \Psi=0,
$$

we obtain

$$
\delta \mathscr{F}=\varepsilon \varphi_{0} \cdot \int_{V} \frac{\partial W}{\partial x} \mathrm{~d} V
$$

Utilizing this in 17, along with the arbitrariness of $\varphi_{0}$, we conclude that

$$
\int_{V} \frac{\partial W}{\partial x} \mathrm{~d} V=\int_{S} t \mathrm{~d} S
$$

In equilibrium this must hold for all $V$, no matter what form the stress vector $t$ takes. Writing this in the manner

$$
\int_{V} \boldsymbol{F} \mathrm{d} V+\int_{S} \boldsymbol{t} \mathrm{d} S=\mathbf{0}, \quad \boldsymbol{F}=-\frac{\partial W}{\partial x},
$$

we see that here $-\partial W / \partial x$ plays the role of a body force, which for $W$ as in 2 is given by

$$
\boldsymbol{F}=-\frac{\partial W}{\partial x}=-U \nabla \rho_{\mathrm{f}}-\rho_{\mathrm{m}} g \nabla h
$$

By the definition of the height function $h, \nabla h$ is just a unit vector in the vertical direction.

An infinitesimal rigid rotation can be produced via

$$
\begin{aligned}
x^{*} & =o+\mathbf{R}(\varepsilon)(x-o), \quad \mathbf{R}(\varepsilon)=\mathbf{I}+\varepsilon \mathbf{W}_{0}+o(\varepsilon), \quad \mathbf{W}_{0} \in \operatorname{Skew}(\mathscr{V}) \\
& =x+\varepsilon \mathbf{W}_{0}(x-o)+o(\varepsilon)=x+\varepsilon \boldsymbol{w}_{0} \times \boldsymbol{r}(x)+o(\varepsilon),
\end{aligned}
$$

where $\boldsymbol{w}_{0}$ is the axial vector of the skew-symmetric tensor $\mathbf{W}_{0}$ and $\boldsymbol{r}(x)=x-o$, the position vector of the point $x$, and

$\boldsymbol{n}^{*}\left(x^{*}\right)=\mathbf{R}(\varepsilon) \boldsymbol{n}(x)=\boldsymbol{n}(x)+\varepsilon \mathbf{W}_{0} \boldsymbol{n}(x)+o(\varepsilon)=\boldsymbol{n}(x)+\varepsilon \boldsymbol{w}_{0} \times \boldsymbol{n}(x)+o(\varepsilon), U^{*}\left(x^{*}\right)=U(x)$. 
From this we obtain

$$
\boldsymbol{\delta} \mathscr{F}=\varepsilon \mathbf{W}_{0} \cdot \int_{V}\left(\frac{\partial W}{\partial x} \otimes \boldsymbol{r}+\frac{\partial W_{\mathrm{H}}}{\partial \boldsymbol{n}} \otimes \boldsymbol{n}\right) \mathrm{d} V=\boldsymbol{\varepsilon} \boldsymbol{w}_{0} \cdot \int_{V}\left(\boldsymbol{r} \times \frac{\partial W}{\partial x}+\boldsymbol{n} \times \frac{\partial W_{\mathrm{H}}}{\partial \boldsymbol{n}}\right) \mathrm{d} V .
$$

Utilizing this in (17), together with $\varphi=\boldsymbol{w}_{0} \times \boldsymbol{r}, \boldsymbol{\psi}=\boldsymbol{w}_{0} \times \boldsymbol{n}$, and the arbitrariness of $\boldsymbol{w}_{0}$, we obtain

$$
\int_{V}\left(\boldsymbol{r} \times \frac{\partial W}{\partial x}+\boldsymbol{n} \times \frac{\partial W_{\mathrm{H}}}{\partial \boldsymbol{n}}\right) \mathrm{d} V=\int_{S}(\boldsymbol{r} \times \boldsymbol{t}+\boldsymbol{n} \times \boldsymbol{s}) \mathrm{d} S .
$$

As before, this can be written in a form identical to the classical case:

$$
\int_{V}(\boldsymbol{r} \times \boldsymbol{F}+\boldsymbol{n} \times \boldsymbol{G}) \mathrm{d} V+\int_{S}(\boldsymbol{r} \times \boldsymbol{t}+\boldsymbol{n} \times \boldsymbol{s}) \mathrm{d} S=\mathbf{0}, \quad \boldsymbol{F}=-\frac{\partial W}{\partial x}, \quad \boldsymbol{G}=-\frac{\partial W_{\mathrm{H}}}{\partial \boldsymbol{n}},
$$

with $-\partial W / \partial x$ playing the role of the body force (as before) and $-\partial W_{\mathrm{H}} / \partial \boldsymbol{n}$ playing the role of the generalized force acting on $\boldsymbol{n}$. With $W_{\mathrm{H}}$ as in (8), $\boldsymbol{G}$ takes the form

$$
\boldsymbol{G}=-\frac{\partial W_{\mathrm{H}}}{\partial \boldsymbol{n}}=\mu_{0} \Delta \chi(\boldsymbol{H} \cdot \boldsymbol{n}) \boldsymbol{H},
$$

which is consistent with [55, Sec. 3.1.4] and [56, Secs. 2.4.2 Remark (ii), 4.2]. The body couple and couple stress vector are usually denoted

$$
K:=n \times G, \quad l:=n \times s .
$$

See [41, Sec. II.A] or [42, Sec. 2] or [56, Sec. 2.4]. Using the expressions for the magnetic induction $\boldsymbol{B}$ and magnetization $\boldsymbol{M}$ in $(9)$, which are valid for a material in a uniaxial nematic liquid crystal phase, we see that

$$
\boldsymbol{K}=\boldsymbol{B} \times \boldsymbol{H}=\mu_{0} \boldsymbol{M} \times \boldsymbol{H},
$$

the "magnetic torque" density [56, Sec. 2.4.2 Remark (ii)]. The expressions for $\boldsymbol{F}$ and $\boldsymbol{G}$ in 20) are exactly what one would obtain from the approach of Sec.3.3 after merging the appropriate compatibility potentials into $W$. The electric-field contributions (from $W_{\mathrm{E}}$ ) enter via a different route, as we shall see.

In our context, then, the conservation laws for linear momentum (force balance) and angular momentum (torque balance) are given in integral form by (18) and 20]. It will be seen below that $\boldsymbol{t}$ and $\boldsymbol{l}$ can be expressed in terms of appropriate tensors:

$$
t=\mathbf{T} \nu, \quad l=\mathbf{L} \nu .
$$

Expressions for the stress tensor $\mathbf{T}$ and couple stress tensor $\mathbf{L}$ will be deduced below. Using these in (18) and 20, combined with the arbitrariness of $V$ and the Divergence Theorem, one can express these balances in point form, precisely as in the classical case ([55, Sec. 3.1.3], [56, Sec. 2.4]):

$$
\boldsymbol{F}+\operatorname{div} \mathbf{T}=\mathbf{0}, \quad \varepsilon_{i j k} T_{k j} \boldsymbol{e}_{i}+\boldsymbol{K}+\operatorname{div} \mathbf{L}=\mathbf{0} .
$$

Here $\varepsilon_{i j k}$ is the Ricci alternator, $\left\{\boldsymbol{e}_{1}, \boldsymbol{e}_{2}, \boldsymbol{e}_{3}\right\}$ is a fixed orthonormal basis for $\mathscr{V}$, and summation over repeated indices is assumed. The condition $211_{1}$ leads to the elimination of the $r$-dependent parts of (20), leaving a balance of couples in $\sqrt{21})_{2}$ that is independent of the origin of the coordinate system. The equations 21] correspond to the static limit of Cauchy's first and second laws of motion for a polar material, as found in [62, Eqns. (205.2), (205.10)] (where the latter is expressed in terms of the skew tensors associated with these 
axial vectors). The first term in the torque balance is the torque density (per unit volume) associated with the skew part of $\mathbf{T}$. It is related to the axial vector of $\operatorname{skew}(\mathbf{T})$ via

$$
\operatorname{axial}(\operatorname{skew}(\mathbf{T}))=\frac{1}{2} \varepsilon_{i j k} T_{k j} e_{i}
$$

as observed in [55, Secs. 2.1.5, 3.1.3]. In the absence of any body couple and couple stress ( $\boldsymbol{K}=\mathbf{0}, \mathbf{L}=\mathbf{0}$ ), the torque balance would simply dictate that $\mathbf{T}$ be symmetric, as is the case in nonpolar materials.

\subsection{Variations and constraints}

To study more general displacements, we use a variational formula of [23, Sec. 37] for an integral functional of a collection of scalar fields of the form

$$
J\left[u_{1}, \ldots, u_{m}\right]=\int_{V} F\left(x, u_{1}, \ldots, u_{m}, \nabla u_{1}, \ldots, \nabla u_{m}\right) \mathrm{d} V .
$$

In the notation of that book, the variations take the form

$$
\begin{aligned}
x^{*} & =\Phi(x, u, \nabla u ; \varepsilon)=x+\varepsilon \varphi(x, u, \nabla u)+o(\varepsilon) \\
u_{i}^{*}\left(x^{*}\right) & =\Psi_{i}(x, u, \nabla u ; \varepsilon)=u_{i}(x)+\varepsilon \psi_{i}(x, u, \nabla u)+o(\varepsilon),
\end{aligned}
$$

where

$$
u=\left(u_{1}, \ldots, u_{m}\right), \quad \nabla u=\left(\nabla u_{1}, \ldots, \nabla u_{m}\right) .
$$

Such variations involve both the independent and dependent variables and are sometimes referred to as "combined" or "generalized" variations. In [56. Sec. 2.4.1], they are termed "non-contemporaneous variations." The expression for $\delta J$ (defined in the same way we have used in the previous section) can be written

$$
\begin{aligned}
\delta J= & \varepsilon \int_{V}\left(\frac{\partial F}{\partial x} \cdot \boldsymbol{\varphi}+\mathbf{T}_{\mathrm{c}} \cdot \nabla \boldsymbol{\varphi}+\frac{\partial F}{\partial u_{i}} \psi_{i}+\frac{\partial F}{\partial \nabla u_{i}} \cdot \nabla u_{i}\right) \mathrm{d} V \\
= & \varepsilon \int_{V}\left\{\left(\frac{\partial F}{\partial x}-\operatorname{div} \mathbf{T}_{\mathrm{c}}\right) \cdot \boldsymbol{\varphi}+\left[\frac{\partial F}{\partial u_{i}}-\operatorname{div}\left(\frac{\partial F}{\partial \nabla u_{i}}\right)\right] \psi_{i}\right\} \mathrm{d} V \\
& +\varepsilon \int_{S}\left[\mathbf{T}_{\mathrm{c}} \boldsymbol{\nu} \cdot \boldsymbol{\varphi}+\left(\frac{\partial F}{\partial \nabla u_{i}} \cdot \boldsymbol{\nu}\right) \psi_{i}\right] \mathrm{d} S
\end{aligned}
$$

where

$$
\mathbf{T}_{\mathrm{c}}=F \mathbf{I}-\nabla u_{1} \otimes \frac{\partial F}{\partial \nabla u_{1}}-\cdots-\nabla u_{m} \otimes \frac{\partial F}{\partial \nabla u_{m}} .
$$

In all formulas, summation over repeated indices is implied.

Though written in a different way, the expression above for $\delta J$ is equivalent to [23, Eqn. (105)] and [56, Eqn. (2.125)]. In [60, Sec.2.d], the tensor $\mathbf{T}_{\mathrm{c}}$ is referred to as the "canonical stress tensor," a term used in field theories in relativistic physics. In one space dimension, such expressions are related to canonical variables and first integrals [23, Sec. 17]. If, for example, $F$ were the strain-energy density of a hyperelastic material, then such a relation $\left(\mathbf{T}_{\mathrm{c}}=\partial F / \partial \nabla \varphi\right)$ would correspond to the relationship between the Piola-Kirchoff stress (or nominal stress) and the deformation gradient in that setting [29, Sec. 28], [47, 
Sec. 4.3.1]. For our free-energy functional (15), with displacements denoted as in (16), the general variational formula above assumes the form

$$
\begin{aligned}
\boldsymbol{\delta} \mathscr{F}= & \varepsilon \int_{V}\left(\frac{\partial W}{\partial x} \cdot \boldsymbol{\varphi}+\mathbf{T}_{\mathrm{c}} \cdot \nabla \boldsymbol{\varphi}+\frac{\partial W}{\partial \boldsymbol{n}} \cdot \boldsymbol{\psi}+\frac{\partial W}{\partial \nabla \boldsymbol{n}} \cdot \nabla \boldsymbol{\psi}+\frac{\partial W}{\partial U} \Psi+\frac{\partial W}{\partial \nabla U} \cdot \nabla \Psi\right) \mathrm{d} V \\
= & \varepsilon \int_{V}\left\{\left(\frac{\partial W}{\partial x}-\operatorname{div} \mathbf{T}_{\mathrm{c}}\right) \cdot \boldsymbol{\varphi}+\left[\frac{\partial W}{\partial \boldsymbol{n}}-\operatorname{div}\left(\frac{\partial W}{\partial \nabla \boldsymbol{n}}\right)\right] \cdot \boldsymbol{\psi}+\left[\frac{\partial W}{\partial U}-\operatorname{div}\left(\frac{\partial W}{\partial \nabla U}\right)\right] \boldsymbol{\Psi}\right\} \mathrm{d} V \\
& +\varepsilon \int_{S}\left[\mathbf{T}_{\mathrm{c}} \boldsymbol{\nu} \cdot \boldsymbol{\varphi}+\left(\frac{\partial W}{\partial \nabla \boldsymbol{n}}\right) \boldsymbol{\nu} \cdot \boldsymbol{\psi}+\left(\frac{\partial W}{\partial \nabla U} \cdot \boldsymbol{\nu}\right) \boldsymbol{\Psi}\right] \mathrm{d} S
\end{aligned}
$$

with

$$
\mathbf{T}_{\mathrm{c}}=W \mathbf{I}-(\nabla \boldsymbol{n})^{T} \frac{\partial W}{\partial \nabla \boldsymbol{n}}-\nabla U \otimes \frac{\partial W}{\partial \nabla U} .
$$

This simply corresponds to 24 and (25) with $m=4$ and the role of $u_{1}, u_{2}, u_{3}$, and $u_{4}$ played by $n_{1}, n_{2}, n_{3}$, and $U$ (where $n_{i}$ are the Cartesian components of $\boldsymbol{n}$ ).

In order to be used in our virtual-work principle, the displacements must be consistent with the constraints of incompressibility and $|\boldsymbol{n}|=1$, which imply $\operatorname{div} \boldsymbol{\varphi}=0$ and $\boldsymbol{\psi} \cdot \boldsymbol{n}=0$ [55. Sec. 2.1.2], [56, Sec. 2.4.2]. These constraints can be implemented in various ways. We impose the incompressibility constraint in the traditional way, by introducing the term $-p \operatorname{div} \varphi$ into the variation, with $p$ the Lagrange multiplier pressure field. The unit-length constraint on the director can be enforced via Lagrange multipliers as well, but here we choose instead to impose it by writing $\boldsymbol{\psi}=\boldsymbol{\chi} \times \boldsymbol{n}$, with $\chi$ an arbitrary rotation vector. With these changes, the constrained variation $\delta \mathscr{F}$ takes the form

$$
\begin{aligned}
\boldsymbol{\delta} \mathscr{F}= & \varepsilon \int_{V}\left\{\left(\frac{\partial W}{\partial x}-\operatorname{div} \mathbf{T}\right) \cdot \boldsymbol{\varphi}+\boldsymbol{n} \times\left[\frac{\partial W}{\partial \boldsymbol{n}}-\operatorname{div}\left(\frac{\partial W}{\partial \nabla \boldsymbol{n}}\right)\right] \cdot \boldsymbol{\chi}+\left[\frac{\partial W}{\partial U}-\operatorname{div}\left(\frac{\partial W}{\partial \nabla U}\right)\right] \Psi\right\} \mathrm{d} V \\
& +\varepsilon \int_{S}\left[\mathbf{T} \boldsymbol{\nu} \cdot \boldsymbol{\varphi}+\boldsymbol{n} \times\left(\frac{\partial W}{\partial \nabla \boldsymbol{n}}\right) \boldsymbol{\nu} \cdot \boldsymbol{\chi}+\left(\frac{\partial W}{\partial \nabla U} \cdot \boldsymbol{\nu}\right) \Psi\right] \mathrm{d} S
\end{aligned}
$$

with

$$
\mathbf{T}=\mathbf{T}_{\mathrm{c}}-p \mathbf{I}=(W-p) \mathbf{I}-(\nabla \boldsymbol{n})^{T} \frac{\partial W}{\partial \nabla \boldsymbol{n}}-\nabla U \otimes \frac{\partial W}{\partial \nabla U} .
$$

At this stage, the pressure field is undetermined. Using the general form for the constrained variation $\delta \mathscr{F}$ above in the virtual-work principle (17), along with the arbitrariness of $\varphi, \chi$, $\Psi$, and the subregion $V$, we obtain the following conditions (to be satisfied in equilibrium):

$$
\begin{array}{cl}
\frac{\partial W}{\partial x}-\operatorname{div} \mathbf{T}=\mathbf{0}, \quad \boldsymbol{n} \times\left[\frac{\partial W}{\partial \boldsymbol{n}}-\operatorname{div}\left(\frac{\partial W}{\partial \nabla \boldsymbol{n}}\right)\right]=\mathbf{0}, & \frac{\partial W}{\partial U}-\operatorname{div}\left(\frac{\partial W}{\partial \nabla U}\right)=0 \\
\boldsymbol{t}=\mathbf{T} \boldsymbol{\nu}, \quad \boldsymbol{n} \times \boldsymbol{s}=\boldsymbol{n} \times\left(\frac{\partial W}{\partial \nabla \boldsymbol{n}}\right) \boldsymbol{\nu}, \quad r=\frac{\partial W}{\partial \nabla U} \cdot \boldsymbol{\nu} .
\end{array}
$$

The field equations 28a recover the local form of the force balance $211_{1}$ along with the Euler-Lagrange equations for the equilibrium director field and electric potential (11a), while (28b) gives the formulas for the generalized force densities and fluxes associated with the influences outside of a fluid element. We note that 28a gives the "strong form" or "point form" of the field equations, and we have tacitly assumed sufficient regularity of the underlying fields to justify the various integrations by parts that we have employed.

One can show that when evaluated on an equilibrium pair $(\boldsymbol{n}, U)$, we necessarily have

$$
\operatorname{div} \mathbf{T}_{\mathrm{c}}=\frac{\partial W}{\partial x} .
$$


This can be verified by direct calculation, using the Euler-Lagrange equations satisfied by $\boldsymbol{n}$ and $U$ together with the relation $(\nabla \boldsymbol{n})^{T} \boldsymbol{n}=\mathbf{0}$ (which is a consequence of $|\boldsymbol{n}|=1$ ). It can also be deduced from the necessary vanishing of the inner variation $\delta_{x} \mathscr{F}$, that is, the unconstrained variation (26) with $\psi=\mathbf{0}, \Psi=0$, and $\operatorname{supp}(\varphi) \subset \operatorname{int}(V)$, which corresponds to a local parallel displacement of $\boldsymbol{n}$ and $U$-see [1, Sec. 2.5]. As a consequence of this, we obtain

$$
\frac{\partial W}{\partial x}-\operatorname{div} \mathbf{T}=\mathbf{0} \Rightarrow \nabla p=\mathbf{0} \Rightarrow p=p_{0}=\text { const },
$$

which is consistent with the fact that in a model that assumes incompressibility, the pressure can only be determined to within an arbitrary constant hydrostatic pressure [61, Sec. 30]. This then provides us with the final form for our stress tensor:

$$
\mathbf{T}=\left(W-p_{0}\right) \mathbf{I}-(\nabla \boldsymbol{n})^{T} \frac{\partial W}{\partial \nabla \boldsymbol{n}}+\boldsymbol{E} \otimes \boldsymbol{D} .
$$

Here we have used $\boldsymbol{E}=-\nabla U$ and $\boldsymbol{D}=\partial W / \partial \nabla U$ to simplify the last term. This is the total stress tensor containing all mechanical and electromagnetic influences, not just contact forces. It is perhaps more appropriate to refer to it as the total momentum-flux tensor. With this definition of $\mathbf{T}$, we readily see that any equilibrium pair $(\boldsymbol{n}, U)$ necessarily satisfies the force balance 21$)_{1}$. The form of the couple stress tensor follows from $(28 b)_{2}$ :

$$
\boldsymbol{l}=\boldsymbol{n} \times \boldsymbol{s}=\boldsymbol{n} \times\left(\frac{\partial W}{\partial \nabla \boldsymbol{n}}\right) \boldsymbol{\nu}=\mathbf{L} \boldsymbol{\nu} \Rightarrow L_{i j}=\varepsilon_{i k l} n_{k} \frac{\partial W}{\partial n_{l, j}},
$$

as in the classical case [55, Eqn. (3.73)], [56, Eqn. (2.165)]. The identification of $\mathbf{T}$ and $\mathbf{L}$ completes the picture foreshadowed at the end of Sec.4.1. The term

$$
r=\frac{\partial W}{\partial \nabla U} \cdot \boldsymbol{\nu}=\boldsymbol{D} \cdot \boldsymbol{\nu}
$$

corresponds to a flux of electric displacement, as in the integral form of the Gauss Law of electrostatics $\left(\int_{S} \boldsymbol{D} \cdot \boldsymbol{\nu} \mathrm{d} S=Q\right.$, with $Q$ the total charge contained in $\left.V\right)$.

\subsection{Satisfaction of force balance and torque balance}

In our development, everything follows from the free energy and associated virtual-work principle. This includes the force balance and torque balance [21, the equilibrium field equations 28a, and the expressions for the stress tensor 29) and couple stress tensor (30). It stands to reason that an equilibrium pair $(\boldsymbol{n}, U)$ (coupled solutions of the Euler-Lagrange equations (11a) should necessarily satisfy the force balance and torque balance, guaranteeing consistency with the hydrostatic limit of the Ericksen-Leslie equations. Satisfaction of the force balance is clear, as we have already observed. Satisfaction of the torque balance is less clear. The traditional approach to verify this is to use an identity due to Ericksen, which follows from the invariance of the distortional elasticity $W_{\mathrm{e}}$ with respect to rigid rotations. This was done first in the absence of external fields in [13] and later generalized to include gravitational and magnetic fields in [14]. Textbook accounts can be found in [55, Sec. 3.1.3] and [56, Sec. 2.4 Remark (i)]. That approach could be followed here, deriving and employing a modified version of "Ericksen's Identity." We choose instead a different approach.

In a simpler variational setting, such balances could be obtained as direct consequences of Noether's Theorem. Broadly stated, this theorem shows that continuous symmetries of 
a system imply conservation laws for that system. These ideas hold a prominent place in variational mechanics, thermodynamics, relativistic physics, and elsewhere. Classical discussions can be found in [11, Ch. 21], [23, Secs. 20, 37.5], and [39, Ch. XI Sec. 20, App. II]. A more modern point of view is presented in [44, Ch. 7], for example. Applications (in general terms) include symmetries with respect to time translation (which can imply conservation of energy), space translation (linear momentum), rotation (angular momentum), and the gauge symmetry of the Maxwell equations (which implies conservation of charge). In continuum mechanics, precursors of these ideas are found in early theories of elasticity-see [61. Sec. 98], where a version of these ideas is referred to as the Cosserat-Toupin Fundamental Equivalence Theorem for hyperelastic materials.

The simplest instance of Noether's Theorem relevant to our interests can be stated as follows. If a functional of the form 22] is invariant under transformations of the form 23 in the sense that $J\left[u_{1}^{*}, \ldots, u_{m}^{*} ; V^{*}\right]=J\left[u_{1}, \ldots, u_{m} ; V\right]$, for all $\varepsilon$ sufficiently small-and this holds for arbitrary subdomains $V$, then

$$
\operatorname{div}\left(\mathbf{T}_{\mathrm{c}}^{T} \boldsymbol{\varphi}+\psi_{i} \frac{\partial F}{\partial \nabla u_{i}}\right)=0
$$

when evaluated on equilibrium fields $u_{1}, \ldots, u_{m}$. Here $\mathbf{T}_{\mathrm{c}}$ is the canonical stress tensor 25]. The result simply follows from the expression for $\delta J$ in 24): invariance implies $\delta J=0$, and equilibrium implies

$$
\frac{\partial F}{\partial x}-\operatorname{div} \mathbf{T}_{\mathrm{c}}=\mathbf{0} \text { and } \frac{\partial F}{\partial u_{i}}-\operatorname{div}\left(\frac{\partial F}{\partial \nabla u_{i}}\right)=0, \quad i=1, \ldots, m,
$$

leaving

$$
0=\int_{S}\left[\mathbf{T}_{\mathrm{c}} \boldsymbol{\nu} \cdot \boldsymbol{\varphi}+\left(\frac{\partial F}{\partial \nabla u_{i}} \cdot \boldsymbol{\nu}\right) \psi_{i}\right] \mathrm{d} S=\int_{S}\left(\mathbf{T}_{\mathrm{c}}^{T} \boldsymbol{\varphi}+\psi_{i} \frac{\partial F}{\partial \nabla u_{i}}\right) \cdot \boldsymbol{\nu} \mathrm{d} S,
$$

with the conclusion following via the Divergence Theorem and the arbitrariness of $V$. See [23, Sec. 37.5]. An equivalent form of the conclusion is

$$
\operatorname{div} \mathbf{T}_{\mathrm{c}} \cdot \boldsymbol{\varphi}+\mathbf{T}_{\mathrm{c}} \cdot \nabla \varphi+\operatorname{div}\left(\frac{\partial F}{\partial \nabla u_{i}}\right) \psi_{i}+\frac{\partial F}{\partial \nabla u_{i}} \cdot \nabla \psi_{i}=0
$$

Note that in the absence of invariance $(\delta J \neq 0)$, the conclusion of Noether's Theorem for this case becomes

$$
\delta J=\varepsilon \int_{V}\left[\operatorname{div} \mathbf{T}_{\mathrm{c}} \cdot \varphi+\mathbf{T}_{\mathrm{c}} \cdot \nabla \varphi+\operatorname{div}\left(\frac{\partial F}{\partial \nabla u_{i}}\right) \psi_{i}+\frac{\partial F}{\partial \nabla u_{i}} \cdot \nabla \psi_{i}\right] \mathrm{d} V,
$$

for all subdomains $V$ and $\varepsilon$ sufficiently small.

A functional of the form

$$
J\left[u_{1}, \ldots, u_{m}\right]=\int_{V} F\left(u_{1}, \ldots, u_{m}, \nabla u_{1}, \ldots, \nabla u_{m}\right) \mathrm{d} V
$$

where $F$ satisfies

$$
F\left(u_{1}, \ldots, u_{m}, \mathbf{R} \nabla u_{1}, \ldots, \mathbf{R} \nabla u_{m}\right)=F\left(u_{1}, \ldots, u_{m}, \nabla u_{1}, \ldots, \nabla u_{m}\right), \quad \forall \mathbf{R} \in S O(\mathscr{V}),
$$

is invariant under rigid translations and rigid rotations. A rigid translation can be expressed

$$
x^{*}=x+\varepsilon \varphi_{0}, u_{i}^{*}\left(x^{*}\right)=u_{i}(x),
$$


in the notation of 23], for which

$$
\varphi=\varphi_{0}, \quad \nabla \varphi=0, \quad \psi_{i}=0, \quad \nabla \psi_{i}=\mathbf{0}
$$

Using these in (31), combined with the arbitrariness of $\varphi_{0}$, gives

$$
\operatorname{div} \mathbf{T}_{\mathrm{c}}=\mathbf{0}
$$

which can be interpreted as a balance of forces (or conservation of linear momentum). In one space dimension, such a relation simply corresponds to a first integral. If $F$ were to contain explicit $x$ dependence, as in 22, then the functional would no longer necessarily be invariant under translation, and this relation would become

$$
\operatorname{div} \mathbf{T}_{\mathrm{c}}=\frac{\partial F}{\partial x}
$$

An infinitesimal rigid rotation can be expressed

$$
x^{*}=x+\varepsilon \mathbf{W}_{0} \boldsymbol{r}(x), \quad u_{i}^{*}\left(x^{*}\right)=u_{i}(x), \quad \text { where } \mathbf{W}_{0} \in \operatorname{Skew}(\mathscr{V}) \text { and } \boldsymbol{r}(x)=x-o,
$$

for which

$$
\varphi=\mathbf{W}_{0} \boldsymbol{r}, \quad \nabla \varphi=\mathbf{W}_{0}, \quad \psi_{i}=0, \quad \nabla \psi_{i}=\mathbf{0} .
$$

Using these in 31 , gives

$$
\operatorname{div} \mathbf{T}_{\mathrm{c}} \cdot \mathbf{W}_{0} \boldsymbol{r}+\mathbf{T}_{\mathrm{c}} \cdot \mathbf{W}_{0}=0 .
$$

Using $\operatorname{div} \mathbf{T}_{\mathrm{c}}=\mathbf{0}$ and the arbitrariness of $\mathbf{W}_{0}$ leads to the conclusion that

$$
\mathbf{T}_{\mathrm{c}} \in \operatorname{Symm}(\mathscr{V}),
$$

which can be viewed as a balance of torques (or conservation of angular momentum). Thus for a frame-indifferent equilibrium model of the form (32), we necessarily have

$$
\operatorname{div} \mathbf{T}_{\mathrm{c}}=\mathbf{0} \text { and } \mathbf{T}_{\mathrm{c}} \in \operatorname{Symm}(\mathscr{V})
$$

when evaluated on fields satisfying the associated strong-form Euler-Lagrange equations. Similar examples, most in the context of relativistic physics, can be found in [23, Sec. 38].

Our liquid-crystal system is not so simple: it is neither translation invariant nor rotation invariant, and it is subject to constraints (incompressibility and $|\boldsymbol{n}|=1$ ). Nevertheless, the same path can be followed to show that conservation of angular momentum (balance of torques) is satisfied by equilibrium fields-linear-momentum conservation (balance of forces) is clear. For $\mathscr{F}[\boldsymbol{n}, U ; V]$ in the form (15) and transformations of the form $[16$, the variation $\delta \mathscr{F}$ is given by (26). When evaluated on an equilibrium pair $(\boldsymbol{n}, U)$, this can be put in the form of the conclusion of Noether's Theorem:

$$
\begin{aligned}
\boldsymbol{\delta} \mathscr{F}= & \varepsilon \int_{V}\left\{\operatorname{div} \mathbf{T}_{\mathrm{c}} \cdot \boldsymbol{\varphi}+\mathbf{T}_{\mathrm{c}} \cdot \nabla \boldsymbol{\varphi}+\left[\operatorname{div}\left(\frac{\partial W}{\partial \nabla \boldsymbol{n}}\right)+\lambda \boldsymbol{n}\right] \cdot \boldsymbol{\psi}\right. \\
& \left.+\frac{\partial W}{\partial \nabla \boldsymbol{n}} \cdot \nabla \boldsymbol{\psi}+\operatorname{div}\left(\frac{\partial W}{\partial \nabla U}\right) \Psi+\frac{\partial W}{\partial \nabla U} \cdot \nabla \Psi\right\} \mathrm{d} V,
\end{aligned}
$$

for all subdomains $V$ and $\varepsilon$ sufficiently small. For an infinitesimal rigid rotation of the form

$$
x^{*}=x+\varepsilon \mathbf{W}_{0} \boldsymbol{r}(x), \quad \boldsymbol{n}^{*}\left(x^{*}\right)=\boldsymbol{n}(x)+\varepsilon \mathbf{W}_{0} \boldsymbol{n}(x), \quad U^{*}\left(x^{*}\right)=U(x), \quad \mathbf{W}_{0} \in \operatorname{Skew}(\mathscr{V}),
$$


we have already obtained in Sec.4.1 that

$$
\boldsymbol{\delta} \mathscr{F}=\varepsilon \mathbf{W}_{0} \cdot \int_{V}\left(\frac{\partial W}{\partial x} \otimes \boldsymbol{r}+\frac{\partial W_{\mathrm{H}}}{\partial \boldsymbol{n}} \otimes \boldsymbol{n}\right) \mathrm{d} V .
$$

For such a transformation, we have

$$
\boldsymbol{\varphi}=\mathbf{W}_{0} \boldsymbol{r}, \nabla \boldsymbol{\varphi}=\mathbf{W}_{0}, \boldsymbol{\psi}=\mathbf{W}_{0} \boldsymbol{n}, \nabla \boldsymbol{\psi}=\mathbf{W}_{0} \nabla \boldsymbol{n}, \Psi=0, \nabla \Psi=\mathbf{0},
$$

which when substituted into 33 gives

$$
\begin{aligned}
\boldsymbol{\delta} \mathscr{F} & =\varepsilon \int_{V}\left\{\operatorname{div} \mathbf{T}_{\mathrm{c}} \cdot \mathbf{W}_{0} \boldsymbol{r}+\mathbf{T}_{\mathrm{c}} \cdot \mathbf{W}_{0}+\left[\operatorname{div}\left(\frac{\partial W}{\partial \nabla \boldsymbol{n}}\right)+\lambda \boldsymbol{n}\right] \cdot \mathbf{W}_{0} \boldsymbol{n}+\frac{\partial W}{\partial \nabla \boldsymbol{n}} \cdot \mathbf{W}_{0} \nabla \boldsymbol{n}\right\} \mathrm{d} V \\
& =\varepsilon \mathbf{W}_{0} \cdot \int_{V}\left[\operatorname{div} \mathbf{T}_{\mathrm{c}} \otimes \boldsymbol{r}+\mathbf{T}_{\mathrm{c}}+\operatorname{div}\left(\frac{\partial W}{\partial \nabla \boldsymbol{n}}\right) \otimes \boldsymbol{n}+\frac{\partial W}{\partial \nabla \boldsymbol{n}}(\nabla \boldsymbol{n})^{T}\right] \mathrm{d} V .
\end{aligned}
$$

Equating these two expressions for $\delta \mathscr{F}$ and using the fact that $\operatorname{div} \mathbf{T}_{\mathrm{c}}=\partial W / \partial x$, combined with the arbitrariness of $\varepsilon, V$, and $\mathbf{W}_{0}$, gives

$$
\mathbf{T}_{\mathrm{c}}-\frac{\partial W_{\mathrm{H}}}{\partial \boldsymbol{n}} \otimes \boldsymbol{n}+\frac{\partial W}{\partial \nabla \boldsymbol{n}}(\nabla \boldsymbol{n})^{T}+\operatorname{div}\left(\frac{\partial W}{\partial \nabla \boldsymbol{n}}\right) \otimes \boldsymbol{n} \in \operatorname{Symm}(\mathscr{V})
$$

and the above remains true with $\mathbf{T}_{\mathrm{c}}$ replaced by $\mathbf{T}$ (since they differ by the symmetric tensor $\left.p_{0} \mathbf{I}\right)$. Expressing in terms of Cartesian components and equating the skew part of the expression above to zero, we find this to be equivalent to

$$
\varepsilon_{i j k} T_{k j}-\varepsilon_{i j k} \frac{\partial W_{\mathrm{H}}}{\partial n_{k}} n_{j}+\frac{\partial}{\partial x_{l}}\left(\varepsilon_{i j k} \frac{\partial W}{\partial n_{k, l}} n_{j}\right)=0,
$$

which is precisely $(21)_{2}$. Thus an equilibrium pair $(\boldsymbol{n}, U)$ necessarily satisfies balance of torques, in addition to balance of forces, and therefore is consistent with the hydrostatic limit of the Ericksen-Leslie equations (properly formulated with a coupled electric field).

The total stress tensor $\mathbf{T}$ in 29 contains both mechanical and electromagnetic influences. The contribution to the torque density $\varepsilon_{i j k} T_{k j} e_{i}$ from the term $\boldsymbol{E} \otimes \boldsymbol{D}$ in $\mathbf{T}$,

$$
\varepsilon_{i j k}(\boldsymbol{E} \otimes \boldsymbol{D})_{k j}=\varepsilon_{i j k} E_{k} D_{j}=(\boldsymbol{D} \times \boldsymbol{E})_{i},
$$

admits an easy interpretation. The expression $\boldsymbol{D} \times \boldsymbol{E}$ is referred to as the "dielectric torque" [34. Sec. 8.4]. As a consequence of the relation $\boldsymbol{D}=\varepsilon_{0} \boldsymbol{E}+\boldsymbol{P}$, we obtain

$$
\boldsymbol{D} \times \boldsymbol{E}=\boldsymbol{P} \times \boldsymbol{E},
$$

and we see that this terms gives the couple per unit volume exerted by the electric field on the polarization, generalizing the formula for the torque on a point dipole in an external field $\boldsymbol{p}_{0} \times \boldsymbol{E}_{\text {ext }}\left(x_{0}\right)$ [57, Sec. 3.9]. In the case of a uniaxial liquid crystal with no flexoelectricity taken into account $\left(\boldsymbol{D}=\varepsilon \boldsymbol{E}, \varepsilon=\varepsilon_{0}\left[\varepsilon_{\perp} \mathbf{I}+\Delta \varepsilon(\boldsymbol{n} \otimes \boldsymbol{n})\right]\right)$, this becomes the familiar expression

$$
\boldsymbol{D} \times \boldsymbol{E}=\varepsilon_{0} \Delta \varepsilon(\boldsymbol{E} \cdot \boldsymbol{n})(\boldsymbol{n} \times \boldsymbol{E}),
$$

which strives to rotate $\boldsymbol{n}$ into alignment parallel to $\pm \boldsymbol{E}$ (if $\Delta \varepsilon>0$ ), perpendicular to $\boldsymbol{E}$ (if $\Delta \varepsilon<0)$. We note that for an isotropic linear dielectric $(\boldsymbol{D}=\varepsilon \boldsymbol{E}, \varepsilon$ a scalar field), we would have $\boldsymbol{D} \times \boldsymbol{E}=\mathbf{0}$. 


\subsection{Boundary conditions}

In order to determine the conditions that hold on the segment $\Gamma_{2}$ of the boundary of $\Omega$, we require the variation of the functional associated with the anchoring energy. Let us separate the total free energy $(1)$ into volume and surface parts:

$$
\mathscr{F}[\boldsymbol{n}, U]=\mathscr{F}_{\mathrm{v}}[\boldsymbol{n}, U ; \Omega]+\mathscr{F}_{\mathrm{s}}\left[\boldsymbol{n} ; \Gamma_{2}\right],
$$

where

$$
\mathscr{F}_{\mathrm{v}}[\boldsymbol{n}, U ; \Omega]=\int_{\Omega} W(x, \boldsymbol{n}, \nabla \boldsymbol{n}, U, \nabla U) \mathrm{d} V, \quad \mathscr{F}_{\mathrm{s}}\left[\boldsymbol{n} ; \Gamma_{2}\right]=\int_{\Gamma_{2}} W_{\mathrm{s}}(x, \boldsymbol{n} ; \boldsymbol{\nu}, \boldsymbol{\tau}) \mathrm{d} S .
$$

Using the same perturbations as in $\left[16\right.$, the variation $\delta \mathscr{F}_{\mathrm{s}}$ can be determined by expansion of

$$
\Delta \mathscr{F}_{\mathrm{s}}=\mathscr{F}_{\mathrm{s}}\left[\boldsymbol{n}^{*} ; \Gamma_{2}^{*}\right]-\mathscr{F}_{\mathrm{s}}\left[\boldsymbol{n} ; \Gamma_{2}\right], \quad \Gamma_{2}^{*}=\Phi_{\varepsilon}\left(\Gamma_{2}\right) .
$$

This is now a true "domain variation," as we now allow deformation of the boundary. In order to expand with respect to $\varepsilon$, we require the transformation properties of $\nu, \tau$, and $\mathrm{d} S$, in addition to those for $x$ and $\boldsymbol{n}$ in (16). The additional formulas that we need are given by

$$
\begin{gathered}
\boldsymbol{\nu}^{*}=\boldsymbol{\nu}-\varepsilon \mathbf{P}(\boldsymbol{\nu})(\nabla \boldsymbol{\varphi})^{T} \boldsymbol{\nu}+o(\varepsilon), \boldsymbol{\tau}^{*}=\boldsymbol{\tau}+\varepsilon \mathbf{P}(\boldsymbol{\tau})(\nabla \boldsymbol{\varphi}) \boldsymbol{\tau}+o(\varepsilon), \\
\mathrm{d} S^{*}=[1+\varepsilon \mathbf{P}(\boldsymbol{\nu}) \cdot \nabla \boldsymbol{\varphi}+o(\varepsilon)] \mathrm{d} S .
\end{gathered}
$$

Here $\mathbf{P}(\boldsymbol{\nu})$ and $\mathbf{P}(\boldsymbol{\tau})$ denote the projections transverse to $\boldsymbol{\nu}$ and $\boldsymbol{\tau}$,

$$
\mathbf{P}(\boldsymbol{\nu})=\mathbf{I}-\boldsymbol{\nu} \otimes \boldsymbol{\nu}, \quad \mathbf{P}(\boldsymbol{\tau})=\mathbf{I}-\boldsymbol{\tau} \otimes \boldsymbol{\tau},
$$

and $\nabla \varphi$ denotes limiting values from the interior of $\Omega$. Formulas similar to those for $\nu^{*}$ and $\mathrm{d} S^{*}$ above can be found in [29, Sec. 6] and [63, Sec. 5.2]. The formula for $\tau^{*}$ follows from

$$
\boldsymbol{\tau}^{*}=\frac{\left(\nabla \Phi_{\varepsilon}\right) \boldsymbol{\tau}}{\left|\left(\nabla \Phi_{\varepsilon}\right) \boldsymbol{\tau}\right|}, \quad \nabla \Phi_{\varepsilon}=\mathbf{I}+\varepsilon \nabla \varphi+o(\varepsilon) .
$$

In terms of these, we obtain

$$
\begin{aligned}
\Delta \mathscr{F}_{\mathrm{s}}= & \int_{\Gamma_{2}^{*}} W_{\mathrm{s}}\left(x^{*}, \boldsymbol{n}^{*} ; \boldsymbol{\nu}^{*}, \boldsymbol{\tau}^{*}\right) \mathrm{d} S^{*}-\int_{\Gamma_{2}} W_{\mathrm{s}}(x, \boldsymbol{n} ; \boldsymbol{\nu}, \boldsymbol{\tau}) \mathrm{d} S \\
= & \int_{\Gamma_{2}}\left[W_{\mathrm{s}}\left(x^{*}(x), \boldsymbol{n}^{*} ; \boldsymbol{\nu}^{*}, \boldsymbol{\tau}^{*}\right)-W_{\mathrm{s}}(x, \boldsymbol{n} ; \boldsymbol{\nu}, \boldsymbol{\tau})\right] \mathrm{d} S \\
& +\varepsilon \int_{\Gamma_{2}} W_{\mathrm{s}}(x, \boldsymbol{n} ; \boldsymbol{\nu}, \boldsymbol{\tau})[\mathbf{P}(\boldsymbol{\nu}) \cdot \nabla \boldsymbol{\varphi}] \mathrm{d} S+o(\varepsilon) \\
= & \varepsilon \int_{\Gamma_{2}}\left[\frac{\partial W_{\mathrm{s}}}{\partial x_{\mathrm{S}}} \cdot \boldsymbol{\varphi}+\frac{\partial W_{\mathrm{s}}}{\partial \boldsymbol{n}} \cdot \boldsymbol{\psi}-\frac{\partial W_{\mathrm{s}}}{\partial \boldsymbol{\nu}} \cdot \mathbf{P}(\boldsymbol{\nu})(\nabla \boldsymbol{\varphi})^{T} \boldsymbol{\nu}\right. \\
& \left.+\frac{\partial W_{\mathrm{s}}}{\partial \tau} \cdot \mathbf{P}(\boldsymbol{\tau})(\nabla \boldsymbol{\varphi}) \boldsymbol{\tau}+W_{\mathrm{s}} \mathbf{P}(\boldsymbol{\nu}) \cdot \nabla \boldsymbol{\varphi}\right] \mathrm{d} S+o(\varepsilon),
\end{aligned}
$$

which simplifies to

$$
\delta \mathscr{F}_{\mathrm{s}}=\varepsilon \int_{\Gamma_{2}}\left(\frac{\partial W_{\mathrm{s}}}{\partial x_{\mathrm{S}}} \cdot \boldsymbol{\varphi}+\mathbf{T}_{\mathrm{s}} \cdot \nabla_{\mathrm{S}} \boldsymbol{\varphi}+\frac{\partial W_{\mathrm{s}}}{\partial \boldsymbol{n}} \cdot \boldsymbol{\psi}\right) \mathrm{d} S,
$$


with

$$
\mathbf{T}_{\mathrm{s}}=W_{\mathrm{s}} \mathbf{P}(\boldsymbol{\nu})-\boldsymbol{\nu} \otimes \mathbf{P}(\boldsymbol{\nu}) \frac{\partial W_{\mathrm{s}}}{\partial \boldsymbol{\nu}}+\mathbf{P}(\boldsymbol{\tau}) \frac{\partial W_{\mathrm{s}}}{\partial \tau} \otimes \boldsymbol{\tau} .
$$

Here $\partial W_{\mathrm{s}} / \partial x_{\mathrm{S}}$ and $\nabla_{\mathrm{S}} \varphi$ denote surface gradients. For the small amount of surface calculus that we require, we rely on the formulations in [30,31], [55, Sec. 5.2.3], and [63, Secs. 2.3.6, 5.2]. While the approaches in these references differ somewhat in their details, they agree in the aspects that we require. Related developments relying more on tools of differential geometry and invariant calculus can be found in [45, Chs. XIV-XV], [51, App. A], and [64, Ch. XII]. Considerations of surface anchoring energies depending on only $\boldsymbol{n}$ and $\boldsymbol{\nu}$ can be found in [6,36] and [63, Ch. 5].

Thus for a scalar field $f$ defined on a surface $S$, the surface gradient $\nabla_{\mathrm{S}} f$ is defined as the tangential vector field characterized by

$$
\left.\frac{d}{d t} f(x(t))\right|_{t=0}=\nabla_{\mathrm{S}} f\left(x_{0}\right) \cdot \dot{x}(0),
$$

for all paths $x(t) \subset S$ through $x_{0}=x(0)$. For any extension $\tilde{f}$ of $f$ to a neighborhood of the surface, one can show that

$$
\nabla_{\mathrm{S}} f=\mathbf{P}(\boldsymbol{\nu}) \nabla \tilde{f} .
$$

The notation $\partial W_{\mathrm{s}} / \partial x_{\mathrm{S}}$ above refers to such a vector field. Thus for a simple anchoring energy of the form considered in Sec.2.2. we would have

$$
W_{\mathrm{s}}=-\frac{1}{2} W_{0}(x)\left(\boldsymbol{n} \cdot \boldsymbol{n}_{0}\right)^{2} \Rightarrow \frac{\partial W_{\mathrm{s}}}{\partial x_{\mathrm{S}}}=-\frac{1}{2}\left(\boldsymbol{n} \cdot \boldsymbol{n}_{0}\right)^{2} \nabla_{\mathrm{S}} W_{0},
$$

which implies a tangential force in the direction of decreasing values of $W_{0}$ (zero, if $W_{0}$ is constant). For a vector field $v$ on $S$, the surface gradient $\nabla_{\mathrm{S}} v$ is defined in a similar way:

$$
\left.\frac{d}{d t} \boldsymbol{v}(x(t))\right|_{t=0}=\nabla_{\mathrm{S}} \boldsymbol{v}\left(x_{0}\right) \dot{x}(0) \text {. }
$$

Thus at each point $x \in S, \nabla_{\mathrm{S}} \boldsymbol{v}(x)$ is a linear transformation that maps vectors tangent to $S$ at $x$ into vectors in $\mathscr{V}$. In terms of an extension $\tilde{\boldsymbol{v}}$ of $\boldsymbol{v}$, one can take

$$
\nabla_{\mathrm{S}} \boldsymbol{v}=(\nabla \tilde{\boldsymbol{v}}) \mathbf{P}(\boldsymbol{\nu})
$$

which produces the correct result when applied to a tangent vector and is extended to annihilate any component of a vector in the normal direction. Thus in 34a, we simply take

$$
\nabla_{\mathrm{S}} \boldsymbol{\varphi}=(\nabla \boldsymbol{\varphi}) \mathbf{P}(\boldsymbol{\nu})
$$

again with $\nabla \varphi$ denoting limiting values from $\operatorname{int}(\Omega)$.

To move the derivatives off $\nabla_{\mathrm{S}} \varphi$ in $(34 \mathrm{a})$, we make use of the identity

$$
\operatorname{div}_{\mathrm{S}}\left(\mathbf{L}^{T} \boldsymbol{v}\right)=\mathbf{L} \cdot \nabla_{\mathrm{S}} \boldsymbol{v}+\operatorname{div}_{\mathrm{S}} \mathbf{L} \cdot \boldsymbol{v}
$$

and the Surface Divergence Theorem for a tangential vector field $t$,

$$
\int_{S} \operatorname{div}_{S} t \mathrm{~d} S=\int_{\partial S} \boldsymbol{t} \cdot \boldsymbol{\nu}_{2} \mathrm{~d} s
$$

The identity can be found in [30, Eqn. $\left.(\mathrm{A} 14)_{1}\right]$ and [63, Eqn. (5.42)]; while the Surface Divergence Theorem can be found in [30, App. A], [31, Sec.2], and [63, Sec. 2.3.6]. Here 
$\nu_{2}$ denotes the binormal (unit vector pointing outward from $S$ on the boundary $\partial S$ ), and the surface divergences of a vector field $\boldsymbol{v}$ and tensor field $\mathbf{L}$ are defined

$$
\operatorname{div}_{\mathrm{S}} \boldsymbol{v}=\operatorname{tr}\left(\nabla_{\mathrm{S}} \boldsymbol{v}\right), \quad \operatorname{div}_{\mathrm{S}}\left(\mathbf{L}^{T} \boldsymbol{a}\right)=\operatorname{div}_{\mathrm{S}} \mathbf{L} \cdot \boldsymbol{a}, \forall \boldsymbol{a} \in \mathscr{V},
$$

as in [31, Sec. 2] and [63, Sec. 2.3.6]. In our setting, then,

$$
\operatorname{div}_{\mathrm{S}} \boldsymbol{\varphi}=\mathbf{P}(\boldsymbol{\nu}) \cdot \nabla \boldsymbol{\varphi}
$$

The surface stress tensor $\mathbf{T}_{\mathrm{s}}$ satisfies $\mathbf{T}_{\mathrm{s}} \boldsymbol{\nu}=\mathbf{0}$, and so $\mathbf{T}_{\mathrm{s}}^{T} \boldsymbol{v}$ is tangential for any vector field $\boldsymbol{v}$ on $S\left(\mathbf{T}_{\mathrm{s}}^{T} \boldsymbol{v} \cdot \boldsymbol{\nu}=\boldsymbol{v} \cdot \mathbf{T}_{\mathrm{s}} \boldsymbol{\nu}=0\right)$. The identity and integral formula above combine to give

$$
\int_{\Gamma_{2}} \mathbf{T}_{\mathrm{s}} \cdot \nabla_{\mathrm{S}} \boldsymbol{\varphi} \mathrm{d} S=\int_{\partial \Gamma_{2}} \mathbf{T}_{\mathrm{s}} \nu_{2} \cdot \varphi \mathrm{d} s-\int_{\Gamma_{2}} \operatorname{div}_{\mathrm{S}} \mathbf{T}_{\mathrm{s}} \cdot \varphi \mathrm{d} S
$$

and we obtain the final expression for $\delta \mathscr{F}_{\mathrm{s}}$ :

$$
\delta \mathscr{F}_{\mathrm{s}}=\varepsilon \int_{\Gamma_{2}}\left[\left(\frac{\partial W_{\mathrm{s}}}{\partial x_{\mathrm{S}}}-\operatorname{div}_{\mathrm{S}} \mathbf{T}_{\mathrm{s}}\right) \cdot \boldsymbol{\varphi}+\frac{\partial W_{\mathrm{s}}}{\partial \boldsymbol{n}} \cdot \boldsymbol{\psi}\right] \mathrm{d} S+\varepsilon \int_{\partial \Gamma_{2}} \mathbf{T}_{\mathrm{s}} \boldsymbol{\nu}_{2} \cdot \boldsymbol{\varphi} \mathrm{d} s
$$

Combining 27) (for the case $V=\Omega$ ) and 35], we have

$$
\begin{aligned}
\delta \mathscr{F}= & \varepsilon \int_{\partial \Omega}\left[\mathbf{T} \boldsymbol{\nu} \cdot \boldsymbol{\varphi}+\boldsymbol{n} \times\left(\frac{\partial W}{\partial \nabla \boldsymbol{n}}\right) \boldsymbol{\nu} \cdot \boldsymbol{\chi}+\left(\frac{\partial W}{\partial \nabla U} \cdot \boldsymbol{\nu}\right) \Psi\right] \mathrm{d} S \\
& +\varepsilon \int_{\Gamma_{2}}\left[\left(\frac{\partial W_{\mathrm{s}}}{\partial x_{\mathrm{S}}}-\operatorname{div}_{\mathrm{S}} \mathbf{T}_{\mathrm{s}}\right) \cdot \boldsymbol{\varphi}+\boldsymbol{n} \times \frac{\partial W_{\mathrm{s}}}{\partial \boldsymbol{n}} \cdot \chi\right] \mathrm{d} S+\varepsilon \int_{\partial \Gamma_{2}} \mathbf{T}_{\mathrm{s}} \boldsymbol{\nu}_{2} \cdot \boldsymbol{\varphi} \mathrm{d} s,
\end{aligned}
$$

where $\mathbf{T}$ is as in $(29), \mathbf{T}_{\mathrm{s}}$ is as in $(34 \mathrm{~b})$, and $\delta \mathscr{F}$ above is assumed to be evaluated on an equilibrium pair $(\boldsymbol{n}, U)$ (which is the reason the volume terms in 27) are not present). The virtual-work principle (17) now takes the form

$$
\delta \mathscr{F}=\varepsilon \int_{\partial \Omega}(\boldsymbol{t} \cdot \boldsymbol{\varphi}+\boldsymbol{l} \cdot \boldsymbol{\chi}+r \Psi) \mathrm{d} S+\varepsilon \int_{\partial \Gamma_{2}} \boldsymbol{f} \cdot \boldsymbol{\varphi} \mathrm{d} s
$$

Balancing expressions above and using the arbitrariness of $\varphi, \chi$, and $\Psi$, along with previously identified essential boundary conditions (see the Fig. 1 caption) and natural boundary condition (11b), we can state the full set of conditions that hold on each of the boundary segments.

On $\Gamma_{1}$, both $\boldsymbol{n}$ and $U$ satisfy Dirichlet boundary conditions $\left(\boldsymbol{n}=\boldsymbol{n}_{0}\right.$ and $\left.U=-V / 2\right)$, and we have the relations

$$
\boldsymbol{t}=\mathbf{T} \boldsymbol{\nu}, \quad \boldsymbol{l}=\boldsymbol{n} \times\left(\frac{\partial W}{\partial \nabla \boldsymbol{n}}\right) \boldsymbol{\nu}=\mathbf{L} \boldsymbol{\nu}, \quad r=\frac{\partial W}{\partial \nabla U} \cdot \boldsymbol{\nu}=\boldsymbol{D} \cdot \boldsymbol{\nu}=-\sigma_{\mathrm{f}} .
$$

Here $t$ is the traction from the substrate, $l$ the couple stress exerted by the interface on the director field adjacent to it (with $\mathbf{L}$ as given in (30), and $\sigma_{\mathrm{f}}$ the density of free charge at a point on the surface of the electrode. The minus sign in front of $\sigma_{\mathrm{f}}$ above is due to the fact that $\nu$ is outward from $\Omega$, not outward from the electrode. One can understand the connection between $r, \sigma_{\mathrm{f}}, \Psi$, and work as follows. The quantity $\varepsilon \Psi$ corresponds to an infinitesimal change $\delta U$ in the electric potential $U$ on $\Gamma_{1}$, i.e., an infinitesimal change in the voltage on the lower electrode. The associated work done by the variable voltage source to effect this change can be deduced from the relations for a parallel-plate capacitor:

$$
Q=C V, \quad W=\frac{1}{2} Q V=\frac{1}{2} C V^{2},
$$


where $Q$ is the total charge on the positively charged capacitor plate ( $-Q$ on the opposite plate), $C$ is the capacitance, $V$ is the potential difference, and $W$ is the work (electrostatic energy) to charge the capacitor in an incremental, reversible way (see [49, Sec. 6.6]). The incremental work associated with an incremental change in voltage is thus

$$
\delta W=C V \delta V=Q \delta V,
$$

which is a special case of [40, Eqn. (2.6)]. The coupling between $r=-\sigma_{\mathrm{f}}$ and $\varepsilon \Psi=\delta U$ is reflective of this in a pointwise sense on $\Gamma_{1}$ (one periodic cell of the lower electrode plate).

On $\Gamma_{2}, U$ satisfies $U=V / 2$, while $\boldsymbol{n}$ satisfies the natural boundary condition (11b), and we have the relations

$\boldsymbol{t}=\mathbf{T} \boldsymbol{\nu}+\frac{\partial W_{\mathrm{s}}}{\partial x_{\mathrm{S}}}-\operatorname{div}_{\mathrm{S}} \mathbf{T}_{\mathrm{s}}, \quad \boldsymbol{l}=\mathbf{L} \boldsymbol{\nu}+\boldsymbol{n} \times \frac{\partial W_{\mathrm{s}}}{\partial \boldsymbol{n}}=\boldsymbol{n} \times\left[\left(\frac{\partial W}{\partial \nabla \boldsymbol{n}}\right) \boldsymbol{\nu}+\frac{\partial W_{\mathrm{s}}}{\partial \boldsymbol{n}}\right]=\mathbf{0}, \quad \boldsymbol{f}=\mathbf{T}_{\mathrm{s}} \boldsymbol{\nu}_{2}$,

and $r=-\sigma_{\mathrm{f}}$ (as on $\Gamma_{1}$ ). In contrast to the relation on $\Gamma_{1}$, the stress vector is not necessarily continuous across $\Gamma_{2}$ : the limiting values are $\mathbf{T} \boldsymbol{\nu}$ from the interior versus $\boldsymbol{t}$ above from the exterior. It suffers instead a jump $\left(\partial W_{\mathrm{s}} / \partial x_{\mathrm{S}}-\operatorname{div}_{\mathrm{S}} \mathbf{T}_{\mathrm{s}}\right)$ related to the anchoring energy supported on $\Gamma_{2}$. The behavior is analogous to that of a surface tension, which can lead to a difference in pressure on opposite sides of an interface. The vector $\partial W_{\mathrm{s}} / \partial x_{\mathrm{S}}$ is tangential, while $\operatorname{div}_{S} \mathbf{T}_{\mathrm{s}}$ can have both tangential and normal components. The couple stress vector as well can be discontinuous, with limit $\mathbf{L} \boldsymbol{\nu}$ from inside and $\boldsymbol{l}=\mathbf{0}$ from outside (the vanishing of $\boldsymbol{l}$ a consequence of the natural boundary condition satisfied by $\boldsymbol{n}$ on $\Gamma_{2}$ ). Thus no torque is transmitted to the substrate by the couple stress; it is instead absorbed by the anchoring energy. The quantity $f$ is the force per unit length exerted by the substrate on the boundary of $\Gamma_{2}$. The couple balance on $\Gamma_{2}$ found here is consistent with [36], as conveyed in [6. Eqn. (2.13)]; while the traction balance here contains additional terms not present in those earlier papers (which considered the interface between a liquid crystal and an isotropic fluid), by virtue of the more general form of anchoring energy employed here. In the section that follows, an illustration is given of the forms that these boundary conditions and jumps take for a simple example anchoring energy.

On $\Gamma_{3}$, we have periodic conditions on both $\boldsymbol{n}$ and $U$ (on opposing sides). In terms of the periodic extensions of the fields, these conditions along with those related to the fluxes can be expressed as continuity conditions:

$$
\llbracket \boldsymbol{n} \rrbracket=\mathbf{0}, \quad \llbracket U \rrbracket=0, \quad \llbracket \mathbf{T} \boldsymbol{\nu} \rrbracket=\mathbf{0}, \quad \llbracket \mathbf{L} \boldsymbol{\nu} \rrbracket=\mathbf{0}, \quad \llbracket \boldsymbol{D} \cdot \boldsymbol{\nu} \rrbracket=0,
$$

where $\llbracket \cdot \rrbracket$ denotes the difference between the limit from the exterior of $\Omega$ and the limit from the interior of $\Omega$ at a point on $\Gamma_{3}$.

\subsection{Weak-anchoring boundary condition example}

For the sake of definiteness, we illustrate the boundary conditions and jump conditions on $\Gamma_{2}$ for the prototypical anchoring energy in 10 ,

$$
\begin{aligned}
W_{\mathrm{s}} & =-\frac{1}{2} W_{0}\left(\boldsymbol{n} \cdot \boldsymbol{n}_{0}\right)^{2}, \quad \boldsymbol{n}_{0}=a \boldsymbol{\nu}+b \boldsymbol{\tau}, \quad a^{2}+b^{2}=1 \\
& =-\frac{1}{2} W_{0}\left[a^{2}(\boldsymbol{n} \cdot \boldsymbol{\nu})^{2}+2 a b(\boldsymbol{n} \cdot \boldsymbol{\nu})(\boldsymbol{n} \cdot \boldsymbol{\tau})+b^{2}(\boldsymbol{n} \cdot \boldsymbol{\tau})^{2}\right],
\end{aligned}
$$

with $W_{0}$ a positive constant. This potential conforms to the full nematic symmetry (indifferent with respect to $O(\mathscr{V})$ ). Special cases include weak homeotropic anchoring $(a= \pm 1$, 
$b=0)$ and weak planar anchoring $(a=0, b= \pm 1)$; the general case with both $a$ and $b$ nonzero corresponds to a weak pre-tilt boundary condition. For a planar boundary $\Gamma_{2}$ (as in our model geometry in Fig. 1), with a Cartesian frame $e_{1}, e_{2}, e_{3}$ aligned with $\tau, \tau_{2}, \nu$, the Cartesian components of the surface stress tensor $\mathbf{T}_{\mathrm{s}}$ in $34 \mathrm{~b}$ associated with $W_{\mathrm{s}}$ above are given by

$$
\left[\mathbf{T}_{\mathrm{s}}\right]=-W_{0}\left(\boldsymbol{n} \cdot \boldsymbol{n}_{0}\right)\left[\begin{array}{ccc}
\frac{1}{2}\left(\boldsymbol{n} \cdot \boldsymbol{n}_{0}\right) & 0 & 0 \\
b\left(\boldsymbol{n} \cdot \boldsymbol{\tau}_{2}\right) & \frac{1}{2}\left(\boldsymbol{n} \cdot \boldsymbol{n}_{0}\right) & 0 \\
\boldsymbol{n} \cdot(b \boldsymbol{\nu}-a \boldsymbol{\tau}) & -a\left(\boldsymbol{n} \cdot \boldsymbol{\tau}_{2}\right) & 0
\end{array}\right] .
$$

The extrema of $W_{\mathrm{s}}$ are at $\boldsymbol{n}= \pm \boldsymbol{n}_{0}$ (minima) and $\boldsymbol{n} \perp \boldsymbol{n}_{0}$ (maxima), and at any point on $\Gamma_{2}$ at which $\boldsymbol{n}$ takes on these values, $\mathbf{T}_{\mathrm{s}}$ assumes simple forms:

$$
\boldsymbol{n}= \pm \boldsymbol{n}_{0} \Rightarrow \mathbf{T}_{\mathrm{s}}=-\frac{1}{2} W_{0}\left(\boldsymbol{e}_{1} \otimes \boldsymbol{e}_{1}+\boldsymbol{e}_{2} \otimes \boldsymbol{e}_{2}\right), \quad \boldsymbol{n} \perp \boldsymbol{n}_{0} \Rightarrow \mathbf{T}_{\mathrm{s}}=\mathbf{0} .
$$

It follows that

$$
\boldsymbol{n}= \pm \boldsymbol{n}_{0} \Rightarrow \boldsymbol{f}=\mathbf{T}_{\mathrm{s}} \boldsymbol{\nu}_{2}=-\frac{1}{2} W_{0} \boldsymbol{\nu}_{2},
$$

that is, at any point on $\partial \Gamma_{2}$ at which the director is aligned with the easy axis, the external force per unit length on $\partial \Gamma_{2}$ must be inward and normal. For the model geometry we assume in Fig. 1. these correspond to equal and opposite forces exerted by adjacent periodic cells on each other.

Since $W_{0}$ is assumed constant, we have $\partial W_{\mathrm{s}} / \partial x_{\mathrm{S}}=\mathbf{0}$, and the boundary condition on the stress vector above becomes

$$
\boldsymbol{t}=\mathbf{T} \boldsymbol{\nu}-\operatorname{div}_{\mathrm{S}} \mathbf{T}_{\mathrm{s}},
$$

with

$$
\operatorname{div}_{\mathrm{S}} \mathbf{T}_{\mathrm{s}}=\left(T_{11,1}+T_{12,2}\right) \boldsymbol{e}_{1}+\left(T_{21,1}+T_{22,2}\right) \boldsymbol{e}_{2}+\left(T_{31,1}+T_{32,2}\right) \boldsymbol{e}_{3}
$$

The formulas for these components are not especially illuminating. We give them here, for the record, excluding a common factor of $-W_{0}\left(T_{i j, k}=-W_{0} \widetilde{T}_{i j, k}\right)$ :

$$
\begin{gathered}
\widetilde{T}_{11,1}=\left(\boldsymbol{n} \cdot \boldsymbol{n}_{0}\right)\left(\frac{\partial \boldsymbol{n}}{\partial x_{1}} \cdot \boldsymbol{n}_{0}\right), \quad \widetilde{T}_{12,2}=0 \\
\widetilde{T}_{21,1}=b\left[\left(\boldsymbol{n} \cdot \boldsymbol{n}_{0}\right)\left(\frac{\partial \boldsymbol{n}}{\partial x_{1}} \cdot \boldsymbol{\tau}_{2}\right)+\left(\boldsymbol{n} \cdot \boldsymbol{\tau}_{2}\right)\left(\frac{\partial \boldsymbol{n}}{\partial x_{1}} \cdot \boldsymbol{n}_{0}\right)\right], \quad \widetilde{T}_{22,2}=\left(\boldsymbol{n} \cdot \boldsymbol{n}_{0}\right)\left(\frac{\partial \boldsymbol{n}}{\partial x_{2}} \cdot \boldsymbol{n}_{0}\right) \\
\widetilde{T}_{31,1}=\left(\boldsymbol{n} \cdot \boldsymbol{n}_{0}\right)\left[\frac{\partial \boldsymbol{n}}{\partial x_{1}} \cdot(b \boldsymbol{\nu}-a \boldsymbol{\tau})\right]+[\boldsymbol{n} \cdot(b \boldsymbol{\nu}-a \boldsymbol{\tau})]\left(\frac{\partial \boldsymbol{n}}{\partial x_{1}} \cdot \boldsymbol{n}_{0}\right) \\
\widetilde{T}_{32,2}=-a\left[\left(\boldsymbol{n} \cdot \boldsymbol{n}_{0}\right)\left(\frac{\partial \boldsymbol{n}}{\partial x_{2}} \cdot \boldsymbol{\tau}_{2}\right)+\left(\boldsymbol{n} \cdot \boldsymbol{\tau}_{2}\right)\left(\frac{\partial \boldsymbol{n}}{\partial x_{2}} \cdot \boldsymbol{n}_{0}\right)\right]
\end{gathered}
$$

Some simplifications occur when special cases (such as $\boldsymbol{n}=\boldsymbol{n}_{0}=\boldsymbol{\nu}, \boldsymbol{n} \perp \boldsymbol{n}_{0}=\boldsymbol{\nu}$, etc.) are considered. We conclude that in general $\operatorname{div}_{\mathrm{S}} \mathbf{T}_{\mathrm{S}}$ has nontrivial planar and normal components and that at any point on $\Gamma_{2}$ at which $\partial \boldsymbol{n} / \partial x_{1}=\partial \boldsymbol{n} / \partial x_{2}=\mathbf{0}$, we have $\operatorname{div}_{\mathrm{S}} \mathbf{T}_{\mathrm{s}}=\mathbf{0}$. In particular, for one-dimensional problems (in which fields depend only on $x_{3}$ ), the terms from $W_{\mathrm{s}}$ play no role $\left(\partial W_{\mathrm{s}} / \partial x_{\mathrm{S}}=\operatorname{div}_{\mathrm{S}} \mathbf{T}_{\mathrm{s}}=\mathbf{0}\right)$, and the stress vector suffers no jump across $\Gamma_{2}: t=\mathbf{T} \nu$.

The contribution from our example $W_{\mathrm{s}}$ to the boundary condition on the couple stress vector,

$$
\boldsymbol{l}=\mathbf{L} \boldsymbol{\nu}+\boldsymbol{n} \times \frac{\partial W_{\mathrm{s}}}{\partial \boldsymbol{n}}=\mathbf{0},
$$


is readily obtained from

$$
W_{\mathrm{s}}=-\frac{1}{2} W_{0}\left(\boldsymbol{n} \cdot \boldsymbol{n}_{0}\right)^{2} \Rightarrow \boldsymbol{n} \times \frac{\partial W_{\mathrm{s}}}{\partial \boldsymbol{n}}=-W_{0}\left(\boldsymbol{n} \cdot \boldsymbol{n}_{0}\right)\left(\boldsymbol{n} \times \boldsymbol{n}_{0}\right)
$$

and admits the following interpretation. Here $\nu$ is the outward normal from $\Omega$ and $-\boldsymbol{\nu}$ the outward normal from the substrate. Thus $-\mathbf{L} \boldsymbol{\nu}$ is the torque (per unit area) transmitted to $\boldsymbol{n}$ on $\Gamma_{2}$ from the interior of $\Omega$, and the boundary condition gives a torque balance on $\Gamma_{2}$ that reads

$$
\mathbf{0}=-\mathbf{L} \boldsymbol{\nu}-\boldsymbol{n} \times \frac{\partial W_{\mathrm{s}}}{\partial \boldsymbol{n}}=-\mathbf{L} \boldsymbol{\nu}+W_{0}\left(\boldsymbol{n} \cdot \boldsymbol{n}_{0}\right)\left(\boldsymbol{n} \times \boldsymbol{n}_{0}\right),
$$

with the last term giving the restoring torque from the anchoring potential striving to bring $\boldsymbol{n}$ into alignment with $\pm \boldsymbol{n}_{0}$. If the anchoring on $\Gamma_{2}$ were infinitely strong (the director on $\Gamma_{2}$ fixed to the boundary), then the torque from the interior would be transmitted to the substrate. With weak anchoring, the torque is instead absorbed by the anchoring potential.

\section{Interpretation}

We wish to put our findings in the context of other established results. We ignore, for the moment, issues related to weak anchoring and summarize our results as follows. For a freeenergy functional of a director field $\boldsymbol{n}$ and electric potential $U$ of the form

$$
\mathscr{F}[\boldsymbol{n}, U]=\int_{\Omega} W(x, \boldsymbol{n}, \nabla \boldsymbol{n}, U, \nabla U) \mathrm{d} V,
$$

the coupled Euler-Lagrange equations are given by

$$
\operatorname{div}\left(\frac{\partial W}{\partial \nabla \boldsymbol{n}}\right)-\frac{\partial W}{\partial \boldsymbol{n}}+\lambda \boldsymbol{n}=\mathbf{0}, \quad \operatorname{div}\left(\frac{\partial W}{\partial \nabla U}\right)-\frac{\partial W}{\partial U}=0
$$

the latter equation being equivalent to

$$
\operatorname{div} \boldsymbol{D}=\rho_{\mathrm{f}}, \text { with } \boldsymbol{D}=\varepsilon_{0} \boldsymbol{E}+\boldsymbol{P}, \boldsymbol{E}=-\nabla U
$$

when $W$ is constructed as in Sec.2.1 using (4). An equilibrium pair $(\boldsymbol{n}, U)$ (solutions of the strong-form Euler-Lagrange equations above) necessarily satisfies an appropriate balance of forces

and balance of torques

$$
\operatorname{div} \mathbf{T}+\boldsymbol{F}=\mathbf{0}, \quad \boldsymbol{F}=-\frac{\partial W}{\partial x}
$$

$$
2 \boldsymbol{w}+\boldsymbol{K}+\operatorname{div} \mathbf{L}=\mathbf{0},
$$

where

$$
\begin{gathered}
\mathbf{T}=\left(W-p_{0}\right) \mathbf{I}-(\nabla \boldsymbol{n})^{T} \frac{\partial W}{\partial \nabla \boldsymbol{n}}+\boldsymbol{E} \otimes \boldsymbol{D}, \quad \boldsymbol{w}=\operatorname{axial}(\operatorname{skew}(\mathbf{T}))=\frac{1}{2} \varepsilon_{i j k} T_{k j} \boldsymbol{e}_{i} \\
\boldsymbol{K}=\boldsymbol{n} \times \boldsymbol{G}, \quad \boldsymbol{G}=-\frac{\partial \widetilde{W}}{\partial \boldsymbol{n}}, \quad \mathbf{L}=\varepsilon_{i k l} n_{k} \frac{\partial W}{\partial n_{l, j}} \boldsymbol{e}_{i} \otimes \boldsymbol{e}_{j} .
\end{gathered}
$$

Here $\widetilde{W}$ denotes the collection of all terms included in $W$ that depend on $\boldsymbol{n}$ and are not invariant under rigid rotations, i.e., $\widetilde{W}(\ldots, \mathbf{R} n, \ldots) \neq \widetilde{W}(\ldots, \boldsymbol{n}, \ldots)$ for some $\mathbf{R} \in \operatorname{SO}(\mathscr{V})$. For $W$ as in (2), we have $\widetilde{W}=W_{\mathrm{H}}$, and the electric-field contributions to the torque balance come from skew $(\mathbf{T})$ (and $\mathbf{L}$, if flexoelectric terms are included). These balances assure us that variational equilibrium $(\boldsymbol{\delta} \mathscr{F}=0)$ would be consistent with the hydrostatic limit of the Ericksen-Leslie equations, properly formulated with a coupled electric field. We first compare these results with the "compatibility potential" approach discussed in Sec. 3 above. 
5.1 Contrast with compatibility potential

As discussed in Sec. 3.3 the original ideas of Ericksen are quite sufficient to establish consistency between variational equilibrium equations and Ericksen-Leslie hydrostatics for the cases of gravitational fields and/or magnetic fields. In that setting, the director equilibrium equation is written

$$
\operatorname{div}\left(\frac{\partial W_{\mathrm{e}}}{\partial \nabla \boldsymbol{n}}\right)-\frac{\partial W_{\mathrm{e}}}{\partial \boldsymbol{n}}+\boldsymbol{G}_{1}+\lambda \boldsymbol{n}=\mathbf{0}, \quad \boldsymbol{G}_{1}=\frac{\partial \Psi}{\partial \boldsymbol{n}},
$$

and the force and torque balances

$$
\operatorname{div} \mathbf{T}_{1}+\boldsymbol{F}_{1}=\mathbf{0}, \quad \boldsymbol{F}_{1}=\frac{\partial \Psi}{\partial x}, \quad 2 \boldsymbol{w}_{1}+\boldsymbol{K}_{1}+\operatorname{div} \mathbf{L}_{1}=\mathbf{0},
$$

where

$$
\begin{gathered}
\mathbf{T}_{1}=-p \mathbf{I}-(\nabla \boldsymbol{n})^{T} \frac{\partial W_{\mathrm{e}}}{\partial \nabla \boldsymbol{n}}, \quad p=p_{0}-W_{\mathrm{e}}+\Psi, \quad \boldsymbol{w}_{1}=\operatorname{axial}\left(\operatorname{skew}\left(\mathbf{T}_{1}\right)\right) \\
\boldsymbol{K}_{1}=\boldsymbol{n} \times \boldsymbol{G}_{1}, \quad \mathbf{L}_{1}=\varepsilon_{i k l} n_{k} \frac{\partial W_{\mathrm{e}}}{\partial n_{l, j}} \boldsymbol{e}_{i} \otimes \boldsymbol{e}_{j} .
\end{gathered}
$$

In the case of a combined magnetic field and gravitational field, the compatibility potential can be written

$$
\Psi=\frac{1}{2} \mu_{0} \Delta \chi(\boldsymbol{H} \cdot \boldsymbol{n})^{2}-\rho_{\mathrm{m}} g h=-W_{\mathrm{H}}-\rho_{\mathrm{m}} g h,
$$

and the two approaches can be seen to be equivalent by identifying $W=W_{\mathrm{e}}-\Psi$ and dropping any equations or terms involving $\boldsymbol{E}$ or $\boldsymbol{D}$ or $U$ (which aren't present in the model in this case).

For the case of an electric field without flexoelectric polarization taken into account, we would have

$$
W=W_{\mathrm{e}}-\Psi, \quad \Psi=\frac{1}{2} \boldsymbol{D} \cdot \boldsymbol{E}-\rho_{\mathrm{f}} U, \quad \boldsymbol{D}=\boldsymbol{\varepsilon}(\boldsymbol{n}) \boldsymbol{E}=\varepsilon_{0}\left[\varepsilon_{\perp} \boldsymbol{E}+\Delta \varepsilon(\boldsymbol{E} \cdot \boldsymbol{n}) \boldsymbol{n}\right] .
$$

Here one could proceed with the compatibility-potential approach by analogy with the magnetic-field case, treating $U$ and $\boldsymbol{E}$ as given fields, as originally hinted at by the language in [14] and as suggested in [55, Sec. 3.1.4], [56, Secs. 2.5, 4.2], and [63, Sec. 4.1.1]—though [56. Sec. 4.2] expresses that proceeding in this way "may be subject to more scrutiny." The results one would obtain are consistent to a good degree with those produced by the coupledelectric-field approach we have taken here, though there are some issues that arise. The fields in (36) versus (37) would be related as follows:

$$
\begin{gathered}
\mathbf{T}=\left(W-p_{0}\right) \mathbf{I}-(\nabla \boldsymbol{n})^{T} \frac{\partial W}{\partial \nabla \boldsymbol{n}}+\boldsymbol{E} \otimes \boldsymbol{D}=\mathbf{T}_{1}+\boldsymbol{E} \otimes \boldsymbol{D} \\
\boldsymbol{F}=-\frac{\partial W}{\partial x}=-U \nabla \rho_{\mathrm{f}}, \quad \boldsymbol{F}_{1}=\frac{\partial \Psi}{\partial x}=(\nabla \boldsymbol{E}) \boldsymbol{D}+\rho_{\mathrm{f}} \boldsymbol{E}-U \nabla \rho_{\mathrm{f}} \\
\boldsymbol{w}=\operatorname{axial}(\operatorname{skew}(\mathbf{T}))=\operatorname{axial}\left(\operatorname{skew}\left(\mathbf{T}_{1}\right)\right)+\operatorname{axial}(\operatorname{skew}(\boldsymbol{E} \otimes \boldsymbol{D}))=\boldsymbol{w}_{1}+\frac{1}{2} \boldsymbol{D} \times \boldsymbol{E} \\
\boldsymbol{G}=-\frac{\partial \widetilde{W}}{\partial \boldsymbol{n}}=\mathbf{0}, \quad \boldsymbol{G}_{1}=\frac{\partial \Psi}{\partial \boldsymbol{n}}=\varepsilon_{0} \Delta \boldsymbol{\varepsilon}(\boldsymbol{E} \cdot \boldsymbol{n}) \boldsymbol{E} \\
\boldsymbol{K}=\boldsymbol{n} \times \boldsymbol{G}=\mathbf{0}, \quad \boldsymbol{K}_{1}=\boldsymbol{n} \times \boldsymbol{G}_{1}=\varepsilon_{0} \Delta \varepsilon(\boldsymbol{E} \cdot \boldsymbol{n}) \boldsymbol{n} \times \boldsymbol{E}=\boldsymbol{D} \times \boldsymbol{E} \\
\mathbf{L}=\varepsilon_{i k l} n_{k} \frac{\partial W}{\partial n_{l, j}} \boldsymbol{e}_{i} \otimes \boldsymbol{e}_{j}=\varepsilon_{i k l} n_{k} \frac{\partial W_{\mathrm{e}}}{\partial n_{l, j}} \boldsymbol{e}_{i} \otimes \boldsymbol{e}_{j}=\mathbf{L}_{1} .
\end{gathered}
$$


Using these, one sees that

$$
\begin{aligned}
\operatorname{div} \mathbf{T}+\boldsymbol{F} & =\operatorname{div} \mathbf{T}_{1}+\operatorname{div}(\boldsymbol{E} \otimes \boldsymbol{D})-U \nabla \rho_{\mathrm{f}} \\
& =\operatorname{div} \mathbf{T}_{1}+(\operatorname{div} \boldsymbol{D}) \boldsymbol{E}+(\nabla \boldsymbol{E}) \boldsymbol{D}-U \nabla \rho_{\mathrm{f}} \\
& =\operatorname{div} \mathbf{T}_{1}+\rho_{\mathrm{f}} \boldsymbol{E}+(\nabla \boldsymbol{E}) \boldsymbol{D}-U \nabla \rho_{\mathrm{f}} \\
& =\operatorname{div} \mathbf{T}_{1}+\boldsymbol{F}_{1}
\end{aligned}
$$

and

$$
2 \boldsymbol{w}+\boldsymbol{K}+\operatorname{div} \mathbf{L}=2 \boldsymbol{w}_{1}+\boldsymbol{D} \times \boldsymbol{E}+\mathbf{0}+\operatorname{div} \mathbf{L}_{1}=2 \boldsymbol{w}_{1}+\boldsymbol{K}_{1}+\operatorname{div} \mathbf{L}_{1} .
$$

Thus the force systems $\boldsymbol{F}, \mathbf{T}, \boldsymbol{K}, \mathbf{L}$ and $\boldsymbol{F}_{1}, \mathbf{T}_{1}, \boldsymbol{K}_{1}, \mathbf{L}_{1}$ are equivalent, in the sense that

$$
\int_{V} \boldsymbol{F} \mathrm{d} V+\int_{S} \mathbf{T} \boldsymbol{\nu} \mathrm{d} S=\int_{V} \boldsymbol{F}_{1} \mathrm{~d} V+\int_{S} \mathbf{T}_{1} \boldsymbol{\nu} \mathrm{d} S
$$

and

$\int_{V}(\boldsymbol{r} \times \boldsymbol{F}+\boldsymbol{K}) \mathrm{d} V+\int_{S}(\boldsymbol{r} \times \mathbf{T} \boldsymbol{\nu}+\mathbf{L} \boldsymbol{\nu}) \mathrm{d} S=\int_{V}\left(\boldsymbol{r} \times \boldsymbol{F}_{1}+\boldsymbol{K}_{1}\right) \mathrm{d} V+\int_{S}\left(\boldsymbol{r} \times \mathbf{T}_{1} \boldsymbol{\nu}+\mathbf{L}_{1} \boldsymbol{\nu}\right) \mathrm{d} S$,

for any volume element $V$ (with boundary $S$ ). That is, both systems produce the same net force and the same net torque on all subregions. These relationships reflect the arbitrariness of how one chooses to define the "body force," "body couple," etc. (commented upon in many places).

The main issue that we see with this approach to dealing with electric fields concerns the definition of $\mathbf{T}$ versus $\mathbf{T}_{1}$ and the implications for determining proper boundary tractions. In our development, $\mathbf{T}$ emerges as a total momentum flux tensor, and so the total force per unit area exerted on one of the boundary electrodes is given by

$$
\mathbf{T} \boldsymbol{\nu}=\left(\mathbf{T}_{1}+\boldsymbol{E} \otimes \boldsymbol{D}\right) \boldsymbol{\nu}=\mathbf{T}_{1} \boldsymbol{\nu}+(\boldsymbol{D} \cdot \boldsymbol{\nu}) \boldsymbol{E}=\mathbf{T}_{1} \boldsymbol{\nu}+\sigma_{\mathrm{f}} \boldsymbol{E},
$$

where $\nu$ is the outward normal from the electrode and $\sigma_{\mathrm{f}}$ the surface charge density at a point. This last piece, $\sigma_{\mathrm{f}} \boldsymbol{E}$, is necessary-without it, the electric-field force density on the electrode (from $\mathbf{T}_{1}$ ) is $-\frac{1}{2} \sigma_{\mathrm{f}} \boldsymbol{E}$ instead of the correct value $\frac{1}{2} \sigma_{\mathrm{f}} \boldsymbol{E}$. Thus $\mathbf{T}_{1} \boldsymbol{\nu}$ does not give the correct expression for the stress vector on an electrode, the reason for this being that the term $\boldsymbol{E} \otimes \boldsymbol{D}$ in $\mathbf{T}$ has been put in $\boldsymbol{F}_{1}$ instead (as $\operatorname{div}(\boldsymbol{E} \otimes \boldsymbol{D})$ ). Other issues that one might raise with this approach are that one must append electrostatics $\left(\operatorname{div} \boldsymbol{D}=\rho_{\mathrm{f}}\right)$ as a side condition, instead of having it embedded naturally in the field equations, and the point that the expression $(\nabla \boldsymbol{E}) \boldsymbol{D}$ in $\boldsymbol{F}_{1}$ does not seem to admit a natural interpretation as a body force. One can make a case for the following as a candidate for the total/net electrostatic body force density (in a linear dielectric in equilibrium):

$$
\boldsymbol{F}_{2}=\operatorname{div}\left[\boldsymbol{E} \otimes \boldsymbol{D}-\frac{1}{2}(\boldsymbol{D} \cdot \boldsymbol{E}) \mathbf{I}\right]=\rho_{\mathrm{f}} \boldsymbol{E}+(\nabla \boldsymbol{E}) \boldsymbol{P}-\frac{1}{2} \nabla(\boldsymbol{P} \cdot \boldsymbol{E}) .
$$

This expression is discussed more in what follows. It has the dependencies $\boldsymbol{F}_{2}=\boldsymbol{F}_{2}(x, \boldsymbol{n}, \nabla \boldsymbol{n})$, however, and so it can't be expressed in the form $\boldsymbol{F}_{2}=\partial \Psi / \partial x$, for any $\Psi=\Psi(x, \boldsymbol{n})$. The term that prevents $\boldsymbol{F}_{2}$ from being such a potential field is the last term, $-\frac{1}{2} \nabla(\boldsymbol{P} \cdot \boldsymbol{E})$, which arises from a pressure gradient and would be handled separately in the compatibility potential framework. The case of electric fields with flexoelectricity taken into account does not fit in the compatibility-potential approach at all, and so it is not possible to make a comparison. 


\subsection{Electric field without flexoelectricity}

It is worthwhile to examine more closely and contrast the cases of an electric field without flexoelectric effects taken into account versus an electric field with flexoelectricity, and we do this now. As we have already observed, the former case is the more common and falls under the umbrella of a linear dielectric medium; while the latter case represents a nonlinear medium and also introduces a coupling between the electric-field variables and the gradient of the director field.

\subsubsection{No free-charge distribution}

In the absence of any free-charge distribution (the most common case), the free-energy density would be given by

$$
W=W_{\mathrm{e}}-\frac{1}{2} \boldsymbol{D} \cdot \boldsymbol{E}, \quad \boldsymbol{D}=\boldsymbol{\varepsilon}(\boldsymbol{n}) \boldsymbol{E}, \quad \boldsymbol{E}=-\nabla U,
$$

where the dielectric tensor $\varepsilon$ is as in (5). The field equations (28a) would take the form

$$
\operatorname{div} \mathbf{T}=\mathbf{0}, \quad \boldsymbol{n} \times\left[\operatorname{div}\left(\frac{\partial W_{\mathrm{e}}}{\partial \nabla \boldsymbol{n}}\right)-\frac{\partial W_{\mathrm{e}}}{\partial \boldsymbol{n}}\right]+\boldsymbol{D} \times \boldsymbol{E}=\mathbf{0}, \quad \operatorname{div} \boldsymbol{D}=0,
$$

with the total stress tensor $\mathbf{T}$ given by

$$
\mathbf{T}=-p_{0} \mathbf{I}+\mathbf{T}_{\mathrm{e}}+\mathbf{T}_{\mathrm{M}}, \quad \mathbf{T}_{\mathrm{e}}:=W_{\mathrm{e}} \mathbf{I}-(\nabla \boldsymbol{n})^{T} \frac{\partial W_{\mathrm{e}}}{\partial \nabla \boldsymbol{n}}, \quad \mathbf{T}_{\mathrm{M}}:=\boldsymbol{E} \otimes \boldsymbol{D}-\frac{1}{2}(\boldsymbol{D} \cdot \boldsymbol{E}) \mathbf{I} .
$$

Here again $\boldsymbol{D} \times \boldsymbol{E}$ is the dielectric torque, and we have decomposed $\mathbf{T}$ into the part arising from distortional elasticity and the part arising from the electric field. The tensor $\mathbf{T}_{\mathbf{M}}$ is familiar and is known as the "Minkowski stress tensor" [33, Sec. 6.9].

The effective total force per unit volume due to the electric field is given by

$$
\operatorname{div} \mathbf{T}_{\mathrm{M}}=(\operatorname{div} \boldsymbol{D}) \boldsymbol{E}+(\nabla \boldsymbol{E}) \boldsymbol{D}-\frac{1}{2} \nabla(\boldsymbol{D} \cdot \boldsymbol{E})=(\nabla \boldsymbol{E}) \boldsymbol{P}-\frac{1}{2} \nabla(\boldsymbol{P} \cdot \boldsymbol{E}) .
$$

Here we have used $\operatorname{div} \boldsymbol{D}=0$ and $\boldsymbol{D}=\varepsilon_{0} \boldsymbol{E}+\boldsymbol{P}$ to simplify above. The term $(\nabla \boldsymbol{E}) \boldsymbol{P}$ is familiar and is known as the "Kelvin force" [25], Sec. 13.1], [26, Ch. II, Secs. 5.f, 8.a]. It is the analogue for a polarization distribution of the formula for the force on a dipole $\boldsymbol{p}_{0}$ at a point $x_{0}$ in an external field (to which the dipole does not contribute): $\nabla \boldsymbol{E}_{\text {ext }}\left(x_{0}\right) \boldsymbol{p}_{0}$ [57, Sec. 3.9]. The term $-\frac{1}{2} \nabla(\boldsymbol{P} \cdot \boldsymbol{E})$, which is also found in [26, Eqn. (347)], is less familiar and admits various interpretations. The expression $-\frac{1}{2} \boldsymbol{P} \cdot \boldsymbol{E}$ gives the electrostatic energy density of a polarization in an electric field of its own generation, to which it is linearly coupled [33, Sec. 4.7], [57, Sec.2.10]. This derives from the expression for the energy of a point dipole in an external field: $-\boldsymbol{p}_{0} \cdot \boldsymbol{E}_{\text {ext }}\left(x_{0}\right)$ [49, Sec. 2-8], [57, Sec. 3.9]. Thus the term $-\frac{1}{2} \nabla(\boldsymbol{P} \cdot \boldsymbol{E})$ can be viewed as a pressure-related force per unit volume (the negative gradient of a pressure contribution $\frac{1}{2} \boldsymbol{P} \cdot \boldsymbol{E}$ ) expressing the desire of the material to expand in regions of large polarization density, so as to reduce the local concentration of polarization. The distinctions among the various pressure contributions ("Kelvin pressure," "Helmholtz pressure," etc.) is discussed in [26, Ch. II, Sec. 8.a]. The polarization is not expected to be uniform, and so one would expect some form of contribution to the force from $\nabla \boldsymbol{P}$. The term 
$-\frac{1}{2} \nabla(\boldsymbol{P} \cdot \boldsymbol{E})$ provides that. We note that terms involving gradients of $\boldsymbol{E}$ versus gradients of $\boldsymbol{P}$ are related by various identities, such as

$$
\int_{V}[(\operatorname{div} \boldsymbol{P}) \boldsymbol{E}+(\nabla \boldsymbol{E}) \boldsymbol{P}] \mathrm{d} V=\int_{S}(\boldsymbol{P} \cdot \boldsymbol{\nu}) \boldsymbol{E} \mathrm{d} S
$$

which is valid for any regular subdomain $V$ with boundary $S$. Using this, one can obtain

$$
\int_{V}\left[(\nabla \boldsymbol{E}) \boldsymbol{P}-\frac{1}{2} \nabla(\boldsymbol{P} \cdot \boldsymbol{E})\right] \mathrm{d} V=\int_{V} \rho_{\boldsymbol{P}} \boldsymbol{E} \mathrm{d} V+\int_{S}\left[\sigma_{\boldsymbol{P}} \boldsymbol{E}-\frac{1}{2}(\boldsymbol{P} \cdot \boldsymbol{E}) \boldsymbol{\nu}\right] \mathrm{d} S,
$$

where $\rho_{P}$ and $\sigma_{P}$ are the effective volume and surface charge densities associated with $\boldsymbol{P}$

$$
\rho_{\boldsymbol{P}}:=-\operatorname{div} \boldsymbol{P}, \quad \sigma_{\boldsymbol{P}}:=\boldsymbol{P} \cdot \boldsymbol{\nu} .
$$

\subsubsection{Self-field correction}

There are other analogies and interpretations to attach to the term $-\frac{1}{2} \nabla(\boldsymbol{P} \cdot \boldsymbol{E})$, and one of them concerns the notions of "self energies" and "self fields." Continuous distributions of charge (volume densities and surface densities) are idealizations and embody "self energies," as is illustrated in [33, Sec. 1.11]. For example, the electrostatic energy of a discrete collection of charges $q_{1}, \ldots, q_{n}$ located at points $p_{1}, \ldots, p_{n}$ (in the electric field of their own generation) can be written

$$
W=\frac{1}{2} \sum_{i} q_{i} U_{i}\left(p_{i}\right), \quad U_{i}(x)=\frac{1}{4 \pi \varepsilon_{0}} \sum_{j \neq i} \frac{q_{j}}{\left|x-p_{j}\right|},
$$

while the analogous formula for a volume distribution $\rho$ is

$$
W=\frac{1}{2} \int_{V} \rho U \mathrm{~d} V, \quad U(x)=\frac{1}{4 \pi \varepsilon_{0}} \int_{V} \frac{\rho\left(x^{\prime}\right)}{\left|x-x^{\prime}\right|} \mathrm{d} V^{\prime} .
$$

The electrostatics of discrete systems is filled with such summations, which exclude indices associated with divergent "self energies," while potential theory is filled with improper integrals similar to that above. It makes no sense to exclude $x=x^{\prime}$ from such an integral expression, and the singularity usually causes no difficulty. There are, however, situations in which complications can arise, such as in the case of the "Kelvin cavity conditions" [35, Sec. 6.10], [57. Sec. 3.28], [59. Sec. 6] (in which one tries to compute the electric field inside a polarized material via the negative gradient of an integral similar to that above and finds that the value of the limit characterizing the improper integral depends on the shape of the shrinking cavity). Another area in which trouble can arise concerns the force on a surface charge distribution, which we now recount.

Coulomb's Law states that the force on a point charge $q_{0}$ at a point $x_{0}$ in an external electric field $\boldsymbol{E}_{\text {ext }}$ (to which $q_{0}$ does not contribute) is given by $q_{0} \boldsymbol{E}_{\text {ext }}\left(x_{0}\right)$. On the basis of this, one would expect that the force density on a volume charge distribution $\rho$ would be given by $\rho \boldsymbol{E}$ and the force density on a surface charge distribution $\sigma$ would be $\sigma \boldsymbol{E}$. The first formula is correct; while the second is not. The true force per unit area on $\sigma$ is $\frac{1}{2} \sigma E$, and one can find multiple derivations and explanations of this - the force on an electrode in a parallel-plate capacitor is a special case. In [40, Sec. 5], the force on $\sigma$ is computed using the Maxwell stress tensor; while in [33, Sec. 1.11], it is computed by what amounts to a virtual-work argument. In introductory books on electromagnetism, this discrepancy $(\sigma \boldsymbol{E}$ versus $\left.\frac{1}{2} \sigma \boldsymbol{E}\right)$ is often explained in terms of "self-field corrections." This is the point of view 
taken in [49, Sec. 4-10], where it is argued that since electric charges do not feel that part of the electric field to which they contribute, then the force on a surface charge distribution should be given by

$$
\boldsymbol{F}_{\sigma}=\int_{S} \sigma \boldsymbol{E}^{\prime} \mathrm{d} S, \quad \boldsymbol{E}^{\prime}=\boldsymbol{E}-\boldsymbol{E}_{\text {self }}
$$

where $\boldsymbol{E}$ is the total electric field at a point and $\boldsymbol{E}_{\text {self }}$ is that part of $\boldsymbol{E}$ due to $\sigma$ on d $S$ itself. A limit argument (which is sketched in [49, Sec. 4-10] and can be made rigorous using results from potential theory [37. Ch. VI, Sec. 5]) gives $\boldsymbol{E}_{\text {self }}=\frac{1}{2} \boldsymbol{E}$, and the result follows.

The reason that the force on $\sigma$ requires adjustment while the force on $\rho$ does not can be explained in terms of scaling-here we use simple examples to illustrate results that are valid in more generality. The electric potentials of a volume charge density in a ball of radius $R$ and a surface charge density on a sphere of the same radius are given by

$$
U_{\rho}(x)=\frac{1}{4 \pi \varepsilon_{0}} \int_{B_{R}} \frac{\rho\left(x^{\prime}\right)}{\left|x-x^{\prime}\right|} \mathrm{d} V^{\prime}, \quad U_{\sigma}(x)=\frac{1}{4 \pi \varepsilon_{0}} \int_{S_{R}} \frac{\sigma\left(x^{\prime}\right)}{\left|x-x^{\prime}\right|} \mathrm{d} S^{\prime},
$$

with associated electric fields $\boldsymbol{E}=-\nabla U$, from which one can establish that

$$
U_{\rho}=O\left(R^{2}\right), \quad \boldsymbol{E}_{\rho}=O(R), \quad U_{\sigma}=O(R), \quad \boldsymbol{E}_{\sigma}=O(1), \quad \text { as } R \rightarrow 0 .
$$

Thus the self field associated with $\rho$ is of vanishing importance, while that of $\sigma$ persists in the infinitesimal limit. The analogous expressions for a polarization density $\boldsymbol{P}$ resemble those for $\sigma$, as we now illustrate-we note in addition that $\boldsymbol{P}$ and $\sigma$ have the same physical dimensions (charge per unit area), whereas $\rho$ has the dimensions of charge per unit volume.

The force on a point dipole $\boldsymbol{p}_{0}$ at a point $x_{0}$ in an external field $\boldsymbol{E}_{\text {ext }}$ (to which $\boldsymbol{p}_{0}$ does not contribute) is given by $\nabla \boldsymbol{E}_{\text {ext }}\left(x_{0}\right) \boldsymbol{p}_{0}$ (as we have already indicated above). On the basis of this, one would guess that the force per unit volume on a polarization (sometimes called the "ponderomotive force") would be given by $(\nabla \boldsymbol{E}) \boldsymbol{P}$ (the Kelvin force), and this has been proposed in various places, including [17, Sec. 3.5.B] and [58, Sec. 4.1]. However, the electric potential of a prescribed polarization $\boldsymbol{P}$ in a ball of radius $R$ (as used for illustration with $\rho$ and $\sigma$ above) is

$$
U_{\boldsymbol{P}}(x)=\frac{1}{4 \pi \varepsilon_{0}} \int_{B_{R}} \frac{\boldsymbol{P}\left(x^{\prime}\right) \cdot\left(x-x^{\prime}\right)}{\left|x-x^{\prime}\right|^{3}} \mathrm{~d} V^{\prime},
$$

from which we deduce that

$$
U_{\boldsymbol{P}}=O(R), \quad \boldsymbol{E}_{\boldsymbol{P}}=O(1), \quad \text { as } R \rightarrow 0 .
$$

Thus there is a non-negligible self field, and one would expect that a self-field correction would be needed to produce the proper force density on $\boldsymbol{P}$ (as in the case of a surface charge distribution). While the situation does not seem to be as amenable to direct calculation as the case of a surface charge density, the term $-\frac{1}{2} \nabla(\boldsymbol{P} \cdot \boldsymbol{E})$ is at least a plausible candidate for this correction: $-\frac{1}{2} \boldsymbol{P} \cdot \boldsymbol{E}$ is the self energy of $\boldsymbol{P}$ and the negative gradient $\frac{1}{2} \nabla(\boldsymbol{P} \cdot \boldsymbol{E})$ the associated self field (which must be subtracted as a "correction"). This point of view is reinforced by (40). In fact, if $\boldsymbol{E}$ were parallel to $\boldsymbol{\nu}$ on $S$, then the expression in (40) would simplify exactly as in the case of the force on a surface charge distribution on a conductor:

$$
\boldsymbol{E}=E \boldsymbol{\nu} \Rightarrow \sigma_{P} \boldsymbol{E}-\frac{1}{2}(\boldsymbol{P} \cdot \boldsymbol{E}) \boldsymbol{\nu}=\sigma_{P} \boldsymbol{E}-\frac{1}{2} \sigma_{P} \boldsymbol{E}=\frac{1}{2} \sigma_{P} \boldsymbol{E}
$$

This is just for the sake of illustration; there is no reason to expect $\boldsymbol{E}$ to be parallel to $\boldsymbol{\nu}$ in general-it would only do so at points at which $S$ coincided with or osculated to the 
equipotential surface through the point. As a final note concerning (40), we observe that the expression in the surface integral can be represented

$$
\sigma_{\boldsymbol{P}} \boldsymbol{E}-\frac{1}{2}(\boldsymbol{P} \cdot \boldsymbol{E}) \boldsymbol{\nu}=\mathbf{T}_{\boldsymbol{P}} \boldsymbol{\nu}, \quad \mathbf{T}_{\boldsymbol{P}}:=\boldsymbol{E} \otimes \boldsymbol{P}-\frac{1}{2}(\boldsymbol{P} \cdot \boldsymbol{E}) \mathbf{I},
$$

where $\mathbf{T}_{\boldsymbol{P}}$ is the part of the Minkowski tensor that involves the polarization $\boldsymbol{P}$. This is analogous to the way that the force on a surface charge density on a conductor in vacuo can be calculated using the Maxwell stress tensor, as mentioned earlier.

In any event, one doesn't expect the Kelvin force $(\nabla \boldsymbol{E}) \boldsymbol{P}$ (with $\boldsymbol{E}$ the total macroscopic electric field at a point) to provide the net force per unit volume on a distribution of polarization. The point is emphasized as well in [40. Sec. 16], where the following formula is given for the net force on a dielectric solid inserted into an electric field (for which the sources remain fixed):

$$
\boldsymbol{F}=\int_{\Omega}\left(\nabla \boldsymbol{E}_{\text {ext }}\right) \boldsymbol{P} \mathrm{d} V
$$

The authors there hasten to point out (in a footnote) that $\left(\nabla \boldsymbol{E}_{\text {ext }}\right) \boldsymbol{P}$ does not give the force density at a point. Other influences are present; however, these cancel out when the integration is done over the entire body. Thus it seems that even the most literal extrapolation from $\nabla \boldsymbol{E}_{\text {ext }}\left(x_{0}\right) \boldsymbol{p}_{0}$ fails to give an acceptable force per unit volume on $\boldsymbol{P}$.

\subsubsection{Free-charge distribution included}

If a free-charge distribution is included, then the expressions for $\boldsymbol{D}$ and $\boldsymbol{E}$ above in $(38)$ do not change, but $W$ would now be given by

$$
W=W_{\mathrm{e}}+\rho_{\mathrm{f}} U-\frac{1}{2} \boldsymbol{D} \cdot \boldsymbol{E} .
$$

The field equations would then take the form

$$
\operatorname{div} \mathbf{T}=U \nabla \rho_{\mathrm{f}}, \quad \boldsymbol{n} \times\left[\operatorname{div}\left(\frac{\partial W_{\mathrm{e}}}{\partial \nabla \boldsymbol{n}}\right)-\frac{\partial W_{\mathrm{e}}}{\partial \boldsymbol{n}}\right]+\boldsymbol{D} \times \boldsymbol{E}=\mathbf{0}, \quad \operatorname{div} \boldsymbol{D}=\rho_{\mathrm{f}},
$$

with the total stress tensor $\mathbf{T}$ given by

$$
\mathbf{T}=\left(\rho_{\mathrm{f}} U-p_{0}\right) \mathbf{I}+\mathbf{T}_{\mathrm{e}}+\mathbf{T}_{\mathrm{M}}
$$

with $\mathbf{T}_{\mathrm{e}}$ and $\mathbf{T}_{\mathrm{M}}$ as before in 39 . The net force per unit volume due to electrostatics (in equilibrium) would now given by

$$
\begin{aligned}
\nabla\left(\rho_{\mathrm{f}} U\right)+\operatorname{div} \mathbf{T}_{\mathrm{M}} & =\rho_{\mathrm{f}} \nabla U+U \nabla \rho_{\mathrm{f}}+(\operatorname{div} \boldsymbol{D}) \boldsymbol{E}+(\nabla \boldsymbol{E}) \boldsymbol{D}-\frac{1}{2} \nabla(\boldsymbol{D} \cdot \boldsymbol{E}) \\
& =-\rho_{\mathrm{f}} \boldsymbol{E}+U \nabla \rho_{\mathrm{f}}+\rho_{\mathrm{f}} \boldsymbol{E}+(\nabla \boldsymbol{E}) \boldsymbol{P}-\frac{1}{2} \nabla(\boldsymbol{P} \cdot \boldsymbol{E}) \\
& =U \nabla \rho_{\mathrm{f}}+(\nabla \boldsymbol{E}) \boldsymbol{P}-\frac{1}{2} \nabla(\boldsymbol{P} \cdot \boldsymbol{E}) .
\end{aligned}
$$

The Coulomb force density $\rho_{\mathrm{f}} \boldsymbol{E}$ is balanced by part of the gradient of the $\rho_{\mathrm{f}} U$ term. The other part of that pressure-related term $\left(U \nabla \rho_{\mathrm{f}}\right)$ is balanced by an identical term on the righthand side of the force balance $\operatorname{div} \mathbf{T}=U \nabla \rho_{\mathrm{f}}$ above, similar to the way in which the gravitational force $-\rho_{\mathrm{m}} g \nabla h$ in 19 would be balanced by an appropriate pressure gradient (as would be the case with any body force entering via $-\partial W / \partial x$ ). The last two terms give an 
expression for the force per unit volume on the polarization, and these terms must be balanced by the stresses due to distortional elasticity, as the force balance has now been reduced to

$$
\operatorname{div} \mathbf{T}_{\mathrm{e}}+(\nabla \boldsymbol{E}) \boldsymbol{P}-\frac{1}{2} \nabla(\boldsymbol{P} \cdot \boldsymbol{E})=\mathbf{0}
$$

The term $-U \nabla \rho_{\mathrm{f}}$ represents a force that arises due to the artificial way that the freecharge density enters our model, in which we treat $\rho_{\mathrm{f}}$ as a prescribed (fixed) scalar field. As we have indicated earlier, the more realistic situation in the setting of liquid crystals is for free charges to arise from mobile ionic impurities. In that case, the distribution $\rho_{\mathrm{f}}$ would influence and be influenced by the electric field and potential, which in turn influence and are influenced by the director field, and $\rho_{\mathrm{f}}, U$, and $\boldsymbol{n}$ would need to be computed in a coupled, self-consistent way. Poisson-Boltzmann theory would need to be employed [3, Ch. 7], [34, Sec. 8.5]—we do not pursue this here. While the force $-U \nabla \rho_{\mathrm{f}}$ is thus somewhat artificial in our setting, the pressure gradient associated with $\rho_{\mathrm{f}} U$ properly balances both this force and the Coulomb force on the free charges in equilibrium, as seen above.

The expression $\rho_{\mathrm{f}} \boldsymbol{E}+(\nabla \boldsymbol{E}) \boldsymbol{P}$ can be found in several places in the continuum-mechanics literature as a proposed form for the body force due to an electric field in a dielectric fluid or solid. In [58, Sec. 4.1], for example, a derivation of it is suggested based upon the formulas for the force on a point charge and that on a point dipole in an external electric field. In [17. Sec. 3.5.B], on the other hand, the authors put forward a derivation based on statistical averaging of the microscopic Maxwell-Lorentz equations, along the lines of [26]. The development in [17, Sec. 3.5.B] (once magnetic terms are removed) produces three contributions to the force density: the Coulomb force $\rho_{\mathrm{f}} \boldsymbol{E}$, a force on polarization of the form $(\nabla \boldsymbol{E}) \boldsymbol{P}$, and the negative gradient of a pressure, which is left to be modeled constitutively. For the constitutive theory of electromagnetic fluids later developed [17, Sec. 5.12], the authors incorporate $-\frac{1}{2} \boldsymbol{P} \cdot \boldsymbol{E}$ into the free energy $\left(\frac{1}{2} \boldsymbol{P} \cdot \boldsymbol{E}\right.$ into the pressure), effectively reproducing the third, fourth, and fifth terms in the middle step in 41) above. A somewhat similar statistical-averaging approach can be found in [19].

The authors of [17, 18] acknowledge the arbitrariness of what contributions are put in the "body force" versus the stress tensor [17, Sec. 3.10], and while they choose to take a body force of the form $\rho_{\mathrm{f}} \boldsymbol{E}+(\nabla \boldsymbol{E}) \boldsymbol{P}$ (in the electrostatic setting), when they derive jump conditions at material interfaces and mechanical tractions on boundaries, they move these terms into associated tensors-see [17, Secs. 3.10,3.14]. The relation

$$
\operatorname{div} \mathbf{T}_{\mathrm{M}}=\rho_{\mathrm{f}} \boldsymbol{E}+(\nabla \boldsymbol{E}) \boldsymbol{P}-\frac{1}{2} \nabla(\boldsymbol{P} \cdot \boldsymbol{E})
$$

with the Minkowski tensor $\mathbf{T}_{\mathbf{M}}$ as given in 39 , can in fact be derived as an identity directly from the macroscopic Maxwell equations of electrostatics, using only $\operatorname{curl} \boldsymbol{E}=\mathbf{0}$, $\boldsymbol{D}=\varepsilon_{0} \boldsymbol{E}+\boldsymbol{P}$, and $\operatorname{div} \boldsymbol{D}=\rho_{\mathrm{f}}$ (with no assumptions about how $\boldsymbol{P}$ depends on $\boldsymbol{E}$ ) [7, 19, 20]. By rearranging terms, however, other similar-looking identities can be obtained. The arbitrariness of various such expressions and decompositions (including those involving electromagnetic versus mechanical forces) is emphasized in [7], where the author encourages working with total momentum-flux tensors (as is urged by others as well).

\subsubsection{Representations and tensors in the literature}

The same expressions can be written in different (but equivalent) ways and can be found in different forms in the literature. The relations curl $\boldsymbol{E}=\mathbf{0}$ and $\boldsymbol{D}=\varepsilon_{0} \boldsymbol{E}+\boldsymbol{P}$ imply

$$
(\nabla \boldsymbol{E})^{T}=\nabla \boldsymbol{E} \quad \text { and } \quad(\nabla \boldsymbol{E}) \boldsymbol{D}-\frac{1}{2} \nabla(\boldsymbol{D} \cdot \boldsymbol{E})=(\nabla \boldsymbol{E}) \boldsymbol{P}-\frac{1}{2} \nabla(\boldsymbol{P} \cdot \boldsymbol{E}),
$$


which in the notation of $[19,20]$ is written $\frac{1}{2}[\boldsymbol{D}, \boldsymbol{E}]=\frac{1}{2}[\boldsymbol{P}, \boldsymbol{E}]$, with $[\mathbf{A}, \mathbf{B}]:=(\nabla \mathbf{B})^{T} \mathbf{A}-$ $(\nabla \mathbf{A})^{T} \mathbf{B}$. When combined with the assumption of a linear dielectric $(\boldsymbol{D}=\varepsilon \boldsymbol{E})$ with $\varepsilon^{T}=\varepsilon$, these become

$$
(\nabla \boldsymbol{E}) \boldsymbol{D}-\frac{1}{2} \nabla(\boldsymbol{D} \cdot \boldsymbol{E})=-\frac{1}{2} \varepsilon_{i j, k} E_{i} E_{j} \boldsymbol{e}_{k},
$$

which is the form of the ponderomotive force found in [7, 20]. In a perfect linear dielectric, the dielectric tensor $\varepsilon$ is taken to be real and symmetric [40, Sec. 13], [49, Sec. 15-3]. If the linear medium is isotropic (the dielectric tensor scalar), then the above expression simplifies to $-\frac{1}{2} E^{2} \nabla \varepsilon$, which forms part of the Helmholtz ponderomotive force as found in [25, Sec. 13.1], [26, Ch. II, Sec. 8.a], [40, Sec. 15], [57, Sec. 2.22]. Whether isotropic or not, the force density vanishes if the medium is homogeneous. For the case of a uniaxial nematic liquid crystal (absent flexoelectric effects), we would have a linear, anisotropic dielectric medium

$$
\boldsymbol{D}=\boldsymbol{\varepsilon} \boldsymbol{E}, \quad \boldsymbol{\varepsilon}=\varepsilon_{0}\left[\varepsilon_{\perp} \mathbf{I}+\Delta \varepsilon(\boldsymbol{n} \otimes \boldsymbol{n})\right],
$$

as in [5], and the polarization force above would simplify to

$$
(\nabla \boldsymbol{E}) \boldsymbol{D}-\frac{1}{2} \nabla(\boldsymbol{D} \cdot \boldsymbol{E})=-\varepsilon_{0} \Delta \varepsilon(\boldsymbol{E} \cdot \boldsymbol{n})(\nabla \boldsymbol{n})^{T} \boldsymbol{E} .
$$

As is the case with the general expressions, this force density depends on the gradient of the dielectric tensor, here through the term $\nabla \boldsymbol{n}$, vanishing in regions of uniform orientation $(\boldsymbol{n}=\mathrm{const})$. This force density also vanishes at any points where $\boldsymbol{E}$ is either parallel to $\boldsymbol{n}$ (because of $(\nabla \boldsymbol{n})^{T} \boldsymbol{n}=\mathbf{0}$ ) or perpendicular to $\boldsymbol{n}$ (because of $\boldsymbol{E} \cdot \boldsymbol{n}=\mathbf{0}$ ). We note that this expression is invariant with respect to both $\pm \boldsymbol{n}$ and $\pm \boldsymbol{E}$, as it should be.

The form of the tensor $\mathbf{T}$ that we have derived in $(29)$ is consistent with results in the physics literature, including those found in [25,33,40,57], the closest related results being those found in [40, Sec. 15]. There a dielectric fluid is modeled as a single species of fixed total mass in a fixed volume, with a thermodynamic state that depends on mass density $\rho_{\mathrm{m}}$, temperature $T$, and electric field $\boldsymbol{E}$ at each point. The authors employ what amounts to a virtual-work argument, involving an isothermal deformation at constant electric potential, and deduce the following expression for a general stress tensor (in the notation of that book):

$$
\sigma_{i k}=E_{i} D_{k}+\left[\widetilde{F}-\rho_{\mathrm{m}}\left(\frac{\partial \widetilde{F}}{\partial \rho_{\mathrm{m}}}\right)\right] \delta_{i k} .
$$

Here $\widetilde{F}=\widetilde{F}\left(\rho_{\mathrm{m}}, T, \boldsymbol{E}\right)$ is the Helmholtz free energy per unit volume, and we have transcribed from expressions written in terms of Gaussian units in the book. No additional assumptions about the nature of $\widetilde{F}$ have been made at this point. For an incompressible material (and isochoric deformation), the term involving $\partial \widetilde{F} / \partial \rho_{\mathrm{m}}$ would not arise, and the expression would read

$$
\boldsymbol{\sigma}=\boldsymbol{E} \otimes \boldsymbol{D}+\widetilde{F} \mathbf{I}
$$

in our notation.

For the case of a linear isotropic dielectric ( $\boldsymbol{D}=\varepsilon \boldsymbol{E}, \varepsilon$ a scalar field), $\widetilde{F}$ would be given by

$$
\widetilde{F}=F_{0}\left(\rho_{\mathrm{m}}, T\right)-\frac{1}{2} \varepsilon E^{2},
$$

where $F_{0}$ is the free energy density when no electric field is present. In this case, the stress tensor would take the form

$$
\sigma_{i k}=-P_{0} \delta_{i k}-\frac{1}{2}\left[\varepsilon-\rho_{\mathrm{m}}\left(\frac{\partial \varepsilon}{\partial \rho_{\mathrm{m}}}\right)\right] E^{2} \delta_{i k}+\varepsilon E_{i} E_{k}
$$


where

$$
P_{0}=P_{0}\left(\rho_{\mathrm{m}}, T\right)=-\frac{\partial}{\partial \rho_{\mathrm{m}}^{-1}}\left(\frac{F_{0}}{\rho_{\mathrm{m}}}\right),
$$

the thermodynamic pressure in the absence of an electric field (characterized as the negative of the derivative of the free energy per unit mass with respect to the specific volume $1 / \rho_{\mathrm{m}}$ [26. Ch. II, Sec. 8.a]). Discarding the term $\partial \varepsilon / \partial \rho_{\mathrm{m}}$ (which would not be present in a model of an incompressible material) and generalizing to an anisotropic linear dielectric $(\boldsymbol{D}=\varepsilon \boldsymbol{E}$, $\varepsilon$ a tensor field), the expression can be written

$$
\boldsymbol{\sigma}=\left(W_{\mathrm{E}}-P_{0}\right) \mathbf{I}+\boldsymbol{E} \otimes \boldsymbol{D}, \quad W_{\mathrm{E}}=-\frac{1}{2} \boldsymbol{D} \cdot \boldsymbol{E}, \quad \boldsymbol{D}=\boldsymbol{\varepsilon} \boldsymbol{E} .
$$

This is equivalent to 29) when $W$ does not depend on $\nabla \boldsymbol{n}$ and we make the identification $P_{0}=p_{0}+W_{\mathrm{E}}-W$. The thermodynamic pressure in the absence of an electric field, $P_{0}$, contains the constant hydrostatic pressure $p_{0}$ and all contributions from $W$ apart from $W_{\mathrm{E}}$. Thus except for the contributions due to distortional elasticity and flexoelectric polarization (both of which involve $\nabla \boldsymbol{n}$ ), (29) agrees with [40, Eqn. (15.9)] under the assumption of incompressibility. Similar expressions can be found in [25, Sec. 13] and [57, Secs. 2.21, 2.23], both of which are referenced in the brief discussion in [33, Sec. 6.9].

The Minkowski tensor $\mathbf{T}_{M}$ in (39), which arises in a natural way in our development (in the case of a linear dielectric medium), is one of several tensors found in the electromagnetics literature. Two other prominent ones are the Einstein-Laub tensor

$$
\mathbf{T}_{\mathrm{E}-\mathrm{L}}=\boldsymbol{E} \otimes \boldsymbol{D}-\frac{1}{2} \varepsilon_{0} E^{2} \mathbf{I}
$$

and the Hertz-Abraham tensor, given by the symmetric part of $\mathbf{T}_{\mathrm{E}-\mathrm{L}}$ (see [7, 19, 20] or [17, Sec. 3.6]). Here we are restricting attention to the case of electrostatics-these tensors all have magnetic-field contributions in their general forms. All of these tensors also reduce to the classical Maxwell stress tensor $\varepsilon_{0}\left[\boldsymbol{E} \otimes \boldsymbol{E}-\frac{1}{2} E^{2} \mathbf{I}\right]$ in the absence of matter. The Minkowski and Einstein-Laub tensors are closely related:

$$
\boldsymbol{D}=\varepsilon_{0} \boldsymbol{E}+\boldsymbol{P} \Rightarrow \mathbf{T}_{\mathrm{M}}=\mathbf{T}_{\mathrm{E}-\mathrm{L}}-\frac{1}{2}(\boldsymbol{P} \cdot \boldsymbol{E}) \mathbf{I},
$$

so that

$$
\operatorname{div} \mathbf{T}_{\mathrm{E}-\mathrm{L}}=\rho_{\mathrm{f}} \boldsymbol{E}+(\nabla \boldsymbol{E}) \boldsymbol{P} \text { vs } \operatorname{div} \mathbf{T}_{\mathrm{M}}=\rho_{\mathrm{f}} \boldsymbol{E}+(\nabla \boldsymbol{E}) \boldsymbol{P}-\frac{1}{2} \nabla(\boldsymbol{P} \cdot \boldsymbol{E})
$$

The Einstein-Laub tensor appears in several places in the continuum-mechanics literature on electromagnetics, including [17, Sec. 3.6] and [58, Sec. 4.2], because $\rho_{\mathrm{f}} \boldsymbol{E}+(\nabla \boldsymbol{E}) \boldsymbol{P}$ is the adopted expression for the electrostatic body force in those theories (with $\frac{1}{2} \boldsymbol{P} \cdot \boldsymbol{E}$ relegated to a pressure contribution). The Hertz-Abraham tensor is found in developments that insist on the stress tensors being symmetric.

\subsection{Electric field with flexoelectricity}

The introduction of flexoelectric terms into our model changes some aspects. As previously noted, the material can no longer be viewed as a linear dielectric medium, and the flexoelectric terms also introduce couplings between $\boldsymbol{E}$ and $\nabla \boldsymbol{n}$, in addition to the couplings between $\boldsymbol{E}$ and $\boldsymbol{n}$ already present in the non-flexoelectric case. It is somewhat surprising that this 
has such a small impact on our general development: the basic forms of the equilibrium equations (11a), force and torque balance (21), stress tensor $\mathbf{T}$ (29), and couple stress tensor L (30) all read the same whether flexoelectric terms are present or not. There are, however, some new contributions made by the flexoelectric terms, and we explore them in this section. It is difficult to find in the literature discussions of forces and stresses in liquid-crystal materials subjected to electric fields with flexoelectric effects included. An exception is [16], which treats macroscopic models of liquid crystals as a special case of a "micropolar" continuum mechanics theory.

We set aside the contributions of a free-charge density $\rho_{\mathrm{f}}$, which would play out as already seen in Sec.5.2.3, and consider a free-energy density of the form

$$
\begin{gathered}
W=W_{\mathrm{e}}(\boldsymbol{n}, \nabla \boldsymbol{n})+W_{\mathrm{E}}(\boldsymbol{n}, \nabla \boldsymbol{n}, \nabla U), W_{\mathrm{E}}=-\frac{1}{2} \varepsilon(\boldsymbol{n}) \boldsymbol{E} \cdot \boldsymbol{E}-\boldsymbol{P}_{\mathrm{f}}(\boldsymbol{n}, \nabla \boldsymbol{n}) \cdot \boldsymbol{E}, \quad \boldsymbol{E}=-\nabla U \\
\boldsymbol{\varepsilon}=\varepsilon_{0}\left[\varepsilon_{\perp} \mathbf{I}+\Delta \varepsilon(\boldsymbol{n} \otimes \boldsymbol{n})\right], \quad \boldsymbol{P}_{\mathrm{f}}=e_{\mathrm{s}}(\operatorname{div} \boldsymbol{n}) \boldsymbol{n}+e_{\mathrm{b}}(\boldsymbol{n} \times \operatorname{curl} \boldsymbol{n}), \quad \boldsymbol{D}=\boldsymbol{\varepsilon}(\boldsymbol{n}) \boldsymbol{E}+\boldsymbol{P}_{\mathrm{f}}(\boldsymbol{n}, \nabla \boldsymbol{n}) .
\end{gathered}
$$

The main elements of interest to us (equilibrium Euler-Lagrange equations, stress tensor and force balance, couple stress tensor and torque balance) all follow from this. The equilibrium equation for the electric potential has the general form

$$
\operatorname{div}\left(\frac{\partial W}{\partial \nabla U}\right)-\frac{\partial W}{\partial U}=0
$$

For $W$ as taken above, we have

$$
\frac{\partial W}{\partial \nabla U}=\boldsymbol{\varepsilon} \boldsymbol{E}+\boldsymbol{P}_{\mathrm{f}}=\boldsymbol{D}, \quad \frac{\partial W}{\partial U}=0 \Rightarrow \operatorname{div} \boldsymbol{D}=\mathbf{0} \text { or } \operatorname{div}(\boldsymbol{\varepsilon} \boldsymbol{E})=-\operatorname{div} \boldsymbol{P}_{\mathrm{f}},
$$

with $-\operatorname{div} \boldsymbol{P}_{\mathrm{f}}$ the effective charge density associated with the flexoelectric polarization $\boldsymbol{P}_{\mathrm{f}}$. If we had included a free-charge distribution in the model, then the above would have become

$$
\operatorname{div} \boldsymbol{D}=\rho_{\mathrm{f}} \text { or } \operatorname{div}(\varepsilon \boldsymbol{E})=\rho_{\mathrm{f}}-\operatorname{div} \boldsymbol{P}_{\mathrm{f}}
$$

The equilibrium equation for the director can be written

$$
\operatorname{div}\left(\frac{\partial W}{\partial \nabla \boldsymbol{n}}\right)-\frac{\partial W}{\partial \boldsymbol{n}}+\lambda \boldsymbol{n}=0 .
$$

For our present model, these terms take the forms

$$
\frac{\partial W}{\partial \boldsymbol{n}}=\frac{\partial W_{\mathrm{e}}}{\partial \boldsymbol{n}}-\frac{1}{2} \frac{\partial}{\partial \boldsymbol{n}}[\boldsymbol{\varepsilon}(\boldsymbol{n}) \boldsymbol{E} \cdot \boldsymbol{E}]-\frac{\partial}{\partial \boldsymbol{n}}\left[\boldsymbol{P}_{\mathrm{f}}(\boldsymbol{n}, \nabla \boldsymbol{n}) \cdot \boldsymbol{E}\right]
$$

with

$\frac{\partial}{\partial \boldsymbol{n}}[\boldsymbol{\varepsilon}(\boldsymbol{n}) \boldsymbol{E} \cdot \boldsymbol{E}]=2 \varepsilon_{0} \Delta \varepsilon(\boldsymbol{E} \cdot \boldsymbol{n}) \boldsymbol{E}, \frac{\partial}{\partial \boldsymbol{n}}\left[\boldsymbol{P}_{\mathrm{f}}(\boldsymbol{n}, \nabla \boldsymbol{n}) \cdot \boldsymbol{E}\right]=e_{\mathrm{s}}(\operatorname{div} \boldsymbol{n}) \boldsymbol{E}+e_{\mathrm{b}}(\operatorname{curl} \boldsymbol{n} \times \boldsymbol{E})$,

and

$$
\frac{\partial W}{\partial \nabla \boldsymbol{n}}=\frac{\partial W_{\mathrm{e}}}{\partial \nabla \boldsymbol{n}}-\frac{\partial}{\partial \nabla \boldsymbol{n}}\left[\boldsymbol{P}_{\mathrm{f}}(\boldsymbol{n}, \nabla \boldsymbol{n}) \cdot \boldsymbol{E}\right]
$$

with

$$
\frac{\partial}{\partial \nabla \boldsymbol{n}}\left[\boldsymbol{P}_{\mathrm{f}}(\boldsymbol{n}, \nabla \boldsymbol{n}) \cdot \boldsymbol{E}\right]=e_{\mathrm{s}}(\boldsymbol{n} \cdot \boldsymbol{E}) \mathbf{I}+e_{\mathrm{b}}(\boldsymbol{n} \otimes \boldsymbol{E}-\boldsymbol{E} \otimes \boldsymbol{n}) .
$$


Using these, we can write the Euler-Lagrange equation for the director in the form

$$
\operatorname{div}\left(\frac{\partial W_{\mathrm{e}}}{\partial \nabla \boldsymbol{n}}\right)-\frac{\partial W_{\mathrm{e}}}{\partial \boldsymbol{n}}+\boldsymbol{G}^{\prime}+\lambda \boldsymbol{n}=\mathbf{0}
$$

with

$$
\boldsymbol{G}^{\prime}=\varepsilon_{0} \Delta \varepsilon(\boldsymbol{E} \cdot \boldsymbol{n}) \boldsymbol{E}+\left(e_{\mathrm{s}}+e_{\mathrm{b}}\right)\left[(\operatorname{div} \boldsymbol{n}) \boldsymbol{E}-(\nabla \boldsymbol{n})^{T} \boldsymbol{E}\right]+\left(e_{\mathrm{b}}-e_{\mathrm{s}}\right)(\nabla \boldsymbol{E}) \boldsymbol{n} .
$$

Here we have deliberately segregated the terms arising from distortional elasticity in an attempt to write the equation in a form resembling the classical form (12b). We see that the part associated with the induced polarization $(\varepsilon \boldsymbol{E})$ contributes to the generalized force $G^{\prime}$ in the usual way (the first term of $G^{\prime}$ above coinciding with the first term of $G$ in (14)), while the flexoelectric polarization contributes the second and third terms of $G^{\prime}$ above. The associated couple density on the director field is $\boldsymbol{n} \times \boldsymbol{G}^{\prime}$, the first term of which $\left(\varepsilon_{0} \Delta \varepsilon(\boldsymbol{E}\right.$. $\boldsymbol{n})(\boldsymbol{n} \times \boldsymbol{E}))$ is the familiar dielectric torque. This term is easy to interpret, as it simply strives to rotate $\boldsymbol{n}$ into alignment parallel to the direction of $\pm \boldsymbol{E}$ (for $\Delta \varepsilon>0$ ), perpendicular to $\boldsymbol{E}$ (for $\Delta \varepsilon<0$ ). The second and third terms of $\boldsymbol{n} \times \boldsymbol{G}^{\prime}$ are more difficult to interpret, best left for the analysis of specific systems. We note however the important distinction that the dielectric torque $\varepsilon_{0} \Delta \varepsilon(\boldsymbol{E} \cdot \boldsymbol{n})(\boldsymbol{n} \times \boldsymbol{E})$ is invariant with respect to $\boldsymbol{E}$ versus $-\boldsymbol{E}$, while the torque associated with the flexoelectric polarization reverses when the direction of $\boldsymbol{E}$ is reversed (a characteristic of flexoelectric effects). We point out that $G^{\prime}$ here is not the same as $G$ in $(20)$ and $(36 \mathrm{c})$. The vector field $G^{\prime}$ here is what one obtains if one writes the director equilibrium equation for this case in the classical form $(12 \mathrm{~b}$ ) and $(13)$; whereas $G$ in 20 ) and $36 \mathrm{c}$ gives the body couple $\boldsymbol{K}=\boldsymbol{n} \times \boldsymbol{G}$ as it naturally emerges in the balance of torques in our development 21$)_{2}$ and $36 \mathrm{~b}$ (with the couples from the electric field coming from skew $(\mathbf{T})$ and $\mathbf{L})$.

The force balance has the general form

$$
\operatorname{div} \mathbf{T}-\frac{\partial W}{\partial x}=\mathbf{0}, \quad \mathbf{T}=\left(W-p_{0}\right) \mathbf{I}-(\nabla \boldsymbol{n})^{T} \frac{\partial W}{\partial \nabla \boldsymbol{n}}+\boldsymbol{E} \otimes \boldsymbol{D} .
$$

For the case of interest in this section, the total stress tensor can be written

$$
\mathbf{T}=-p_{0} \mathbf{I}+\mathbf{T}_{\mathrm{e}}-(\nabla \boldsymbol{n})^{T} \frac{\partial W_{\mathrm{E}}}{\partial \nabla \boldsymbol{n}}+W_{\mathrm{E}} \mathbf{I}+\boldsymbol{E} \otimes \boldsymbol{D},
$$

with $\mathbf{T}_{\mathrm{e}}$ as in 39 . The last two terms above contain contributions of Minkowski type plus contributions from the flexoelectric polarization $\boldsymbol{P}_{\mathrm{f}}$ :

$$
W_{\mathrm{E}} \mathbf{I}+\boldsymbol{E} \otimes \boldsymbol{D}=\boldsymbol{E} \otimes \boldsymbol{\varepsilon} \boldsymbol{E}-\frac{1}{2}(\boldsymbol{\varepsilon} \boldsymbol{E} \cdot \boldsymbol{E}) \mathbf{I}+\boldsymbol{E} \otimes \boldsymbol{P}_{\mathrm{f}}-\left(\boldsymbol{P}_{\mathrm{f}} \cdot \boldsymbol{E}\right) \mathbf{I} .
$$

The expression $W_{\mathrm{E}} \mathbf{I}+\boldsymbol{E} \otimes \boldsymbol{D}$ is along the lines of the general expression (43) from [40, Sec. 15], while the coupling between $\boldsymbol{E}$ and $\nabla \boldsymbol{n}$ introduces the term $-(\nabla \boldsymbol{n})^{T} \partial W_{\mathrm{E}} / \partial \nabla \boldsymbol{n}$, which (using (44)) takes the form

$$
-(\nabla \boldsymbol{n})^{T} \frac{\partial W_{\mathrm{E}}}{\partial \nabla \boldsymbol{n}}=e_{\mathrm{s}}(\boldsymbol{n} \cdot \boldsymbol{E})(\nabla \boldsymbol{n})^{T}-e_{\mathrm{b}}(\nabla \boldsymbol{n})^{T} \boldsymbol{E} \otimes \boldsymbol{n} .
$$

As with the torque density associated with $\boldsymbol{P}_{\mathrm{f}}$, the above contribution to the stress is linear in $\boldsymbol{E}$ but properly invariant with respect to $\boldsymbol{n}$ versus $-\boldsymbol{n}$.

As recalled in (36b) and (36c), the torque balance takes the form

$$
\varepsilon_{i j k} T_{k j} \boldsymbol{e}_{i}+\boldsymbol{K}+\operatorname{div} \mathbf{L}=\mathbf{0},
$$


with $\mathbf{T}$ the total stress tensor, $\boldsymbol{K}$ the body couple, and $\mathbf{L}$ the couple stress tensor. For the case of interest in this section, we have $\boldsymbol{K}=\mathbf{0}$, and $\mathbf{T}$ and $\mathbf{L}$ can be written in the forms

$$
\begin{gathered}
\mathbf{T}=\left(W_{\mathrm{e}}+W_{\mathrm{E}}-p_{0}\right) \mathbf{I}-(\nabla \boldsymbol{n})^{T} \frac{\partial W_{\mathrm{e}}}{\partial \nabla \boldsymbol{n}}-(\nabla \boldsymbol{n})^{T} \frac{\partial W_{\mathrm{E}}}{\partial \nabla \boldsymbol{n}}+\boldsymbol{E} \otimes \boldsymbol{D} \\
L_{i j}=\varepsilon_{i k l} n_{k} \frac{\partial W}{\partial n_{l, j}}=\varepsilon_{i k l} n_{k} \frac{\partial W_{\mathrm{e}}}{\partial n_{l, j}}+\varepsilon_{i k l} n_{k} \frac{\partial W_{\mathrm{E}}}{\partial n_{l, j}} .
\end{gathered}
$$

Only the skew part of $\mathbf{T}$ contributes to the torque balance, and the terms involving $W_{\mathrm{e}}$ contain the usual contributions from distortional elasticity. While $\boldsymbol{D}$ is now of the form $\boldsymbol{D}=\boldsymbol{\varepsilon} \boldsymbol{E}+$ $\boldsymbol{P}_{\mathrm{f}}$ (as opposed to $D=\varepsilon \boldsymbol{E}$, in the case of a linear dielectric medium), the contribution from $\boldsymbol{E} \otimes \boldsymbol{D}$ retains the same form:

$$
\varepsilon_{i j k}(\boldsymbol{E} \otimes \boldsymbol{D})_{k j} \boldsymbol{e}_{i}=\boldsymbol{D} \times \boldsymbol{E} .
$$

The newly appearing contributions from $\boldsymbol{P}_{\mathrm{f}}$ again enter by virtue of the coupling between $\boldsymbol{E}$ and $\nabla \boldsymbol{n}$, via $\partial W_{\mathrm{E}} / \partial \nabla \boldsymbol{n}$. From (44) we obtain

$$
-(\nabla \boldsymbol{n})^{T} \frac{\partial W_{\mathrm{E}}}{\partial \nabla \boldsymbol{n}}=e_{\mathrm{s}}(\boldsymbol{n} \cdot \boldsymbol{E})(\nabla \boldsymbol{n})^{T}-e_{\mathrm{b}}(\nabla \boldsymbol{n})^{T} \boldsymbol{E} \otimes \boldsymbol{n},
$$

which can be used to obtain the flexoelectric contribution to the torque density from the skew part of $\mathbf{T}$ :

$$
\varepsilon_{i j k}\left[-(\nabla \boldsymbol{n})^{T} \frac{\partial W_{\mathrm{E}}}{\partial \nabla \boldsymbol{n}}\right]_{k j} \boldsymbol{e}_{i}=e_{\mathrm{s}}(\boldsymbol{n} \cdot \boldsymbol{E}) \varepsilon_{i j k} n_{j, k} \boldsymbol{e}_{i}-e_{\mathrm{b}} \boldsymbol{n} \times(\nabla \boldsymbol{n})^{T} \boldsymbol{E}
$$

The flexoelectric terms also contribute to the couple stress tensor L. Again using (44), we obtain

$$
\varepsilon_{i k l} n_{k} \frac{\partial W_{\mathrm{E}}}{\partial n_{l, j}} \boldsymbol{e}_{i} \otimes \boldsymbol{e}_{j}=-e_{\mathrm{S}}(\boldsymbol{n} \cdot \boldsymbol{E}) \mathbf{W}(\boldsymbol{n})+e_{\mathrm{b}}(\boldsymbol{n} \times \boldsymbol{E}) \otimes \boldsymbol{n},
$$

where $\mathbf{W}(\boldsymbol{n})$ is the skew tensor associated with the vector $\boldsymbol{n}\left(W_{i j}=\varepsilon_{i k j} n_{k}\right)$. At a point on a surface element with normal $\nu$, the above contribution to the couple stress gives

$$
\varepsilon_{i k l} n_{k} \frac{\partial W_{\mathrm{E}}}{\partial n_{l, j}} v_{j} e_{i}=-e_{\mathrm{s}}(\boldsymbol{n} \cdot \boldsymbol{E})(\boldsymbol{n} \times \boldsymbol{\nu})+e_{\mathrm{b}}(\boldsymbol{n} \cdot \boldsymbol{\nu})(\boldsymbol{n} \times \boldsymbol{E}),
$$

which involves an interplay among $\boldsymbol{n}, \boldsymbol{E}$, and $\boldsymbol{\nu}$. The first term strives to rotate $\boldsymbol{n}$ towards $\boldsymbol{\nu}$ (if $-e_{\mathrm{S}}(\boldsymbol{n} \cdot \boldsymbol{E})>0$ ), away from $\boldsymbol{\nu}$ (if $-e_{\mathrm{s}}(\boldsymbol{n} \cdot \boldsymbol{E})<0$ ); while the second term strives to rotate $\boldsymbol{n}$ toward $\boldsymbol{E}$ (if $e_{\mathrm{b}}(\boldsymbol{n} \cdot \boldsymbol{\nu})>0$ ), away from $\boldsymbol{E}$ (if $e_{\mathrm{b}}(\boldsymbol{n} \cdot \boldsymbol{\nu})<0$ ). Both effects reverse when $\boldsymbol{E}$ is reversed, and the flexoelectric coefficients $e_{\mathrm{s}}$ and $e_{\mathrm{b}}$ can be positive or negative [38, Sec. 4.2] - a collection of results from experimental measurements of flexoelectric coefficients can be found in [8, App. A]. These various flexoelectric contributions are somewhat difficult to interpret in general. They involve both forward and inverse influences: director distortion affecting electric fields by induced polarization, and electric fields inducing director distortion (the so-called "inverse flexoelectric effect" [24, Sec. 3.3.2], [38, Sec. 4.3]). 


\section{Conclusions}

We have considered macroscopic models for the equilibrium orientational properties of a nematic liquid crystal and have included all the force fields of general interest (gravitational, magnetic, electric), focusing on the most common case of an electric field arising from electrodes held at constant potential and taking into account the coupling between the liquid crystal director field $\boldsymbol{n}$ and the electric potential field $U$. The starting point for the analysis has been a free energy that is expressed as an integral functional of $\boldsymbol{n}$ and $U$ and which includes a surface anchoring energy on part of the boundary of the region containing the material. From this (and the associated virtual-work principle), we have followed the same paths as taken by Ericksen and Leslie to deduce appropriate expressions for force balance (and stress tensor) and torque balance (and couple stress tensor) corresponding to hydrostatic equilibrium. A main difference here is the inclusion of the electric potential as a state variable to be determined in a coupled, self-consistent way together with the director field.

We have shown that the solutions of the coupled equilibrium Euler-Lagrange equations for $\boldsymbol{n}$ and $U$ necessarily satisfy the force and torque balance laws associated with hydrostatic equilibrium. We have done this by exploiting the analogy with ideas related to Noether's Theorem, which guarantees the satisfaction of appropriate conservation laws when variational models possess continuous symmetries (here related to translations and rotations). When one starts with a well-formed work function (stored-energy function), one should be able to deduce the satisfaction of such balances by variational equilibrium fields. As already observed in [59, Sec. 10] (in the context of an elastic dielectric solid with a stored energy and virtual-work principle): "If the energy principle is used, we see that a single scalar function of the variables of state is sufficient to characterize the mechanical and electrostatic properties of an elastic dielectric completely." This has been borne out here in a similar setting, though involving a complex dielectric fluid instead of an elastic dielectric solid. The issue of "variational compatibility," which confronts versions of related models for liquid crystals in which the forms of external force fields are postulated, here becomes a moot point, as all such fields arise from potentials that are built into the free energy and hydrostatic equilibrium conditions result automatically.

We have considered the three types of boundary conditions typically used in modeling liquid crystal systems: strong anchoring (Dirichlet boundary conditions), weak anchoring (natural boundary conditions associated with anchoring potentials), and periodic boundary conditions. On the part of the boundary subject to weak anchoring, the surface anchoring energy supported there acts somewhat like a surface tension in that it can lead to discontinuities in the stress vector and couple stress vector, in much the same way that a surface tension can lead to a jump in pressure across the interface supporting it. Here there is, in fact, no couple stress exerted by the liquid crystal material on a weak-anchoring substrate- the couple is instead absorbed by the anchoring energy.

We have endeavored to put our results in the context of related results in the physics and continuum-mechanics literatures. Our results are identical to those obtained by Ericksen using the approach of a "compatibility potential" in the cases in which the only force fields involved are gravitational and/or magnetic. In a sense, our development merely provides a generalization of those ideas to cases involving electric fields, in which the coupling between the director field and the electric field is taken into account (and which can involve couplings to $\nabla \boldsymbol{n}$ as well, if flexoelectric effects are included). We have examined carefully the similarities and differences between the coupled-electric-field approach taken here and the approach of treating the electric field (in the non-flexoelectric setting) in a "compatibility potential manner" analogous to the way that a prescribed non-homogeneous magnetic force 
field could be treated. This latter approach, though advocated in certain places, appears to us to have some difficulties, which we have discussed. In general, these various force fields are distinguished by the facts that a gravitational field is a true external field (independent of the state of the material at a point), a magnetic field can be treated as an external field (by virtue of the weakness of the dependence on the material state), while an electric field should not be treated as an external field (since its dependence on the state of the system is non-negligible).

A main result here is the derivation of the stress tensor

$$
\mathbf{T}=\left(W-p_{0}\right) \mathbf{I}-(\nabla \boldsymbol{n})^{T} \frac{\partial W}{\partial \nabla \boldsymbol{n}}+\boldsymbol{E} \otimes \boldsymbol{D},
$$

found in 29 ) and (36c). This tensor embodies the total stress (total momentum flux) associated with mechanical and electromagnetic influences in our setting, that is, in the setting of a macroscopic model for the equilibrium orientational properties of a material in a nematic liquid crystal phase that is incompressible, inhomogeneous, anisotropic, subject to gravitational and/or magnetic and/or electric force fields, and allows for flexoelectric effects as well as a prescribed distribution of free electric charge. This expression for $\mathbf{T}$ agrees with all of the expressions we have been able to find in the literature for all of the special cases for which we have found results. In the absence of an electric field, this tensor coincides with the classical results of Ericksen for the Cauchy stress in a nematic liquid crystal subject to gravitational and/or magnetic fields, as found in the textbook treatments in de Gennes and Prost [24], Sonnet and Virga [55], and Stewart [56]. In the absence of flexoelectricity, electrostatic parts may be segregated into

$$
W_{\mathrm{E}} \mathbf{I}+\boldsymbol{E} \otimes \boldsymbol{D}
$$

where $W_{\mathrm{E}}$ denotes the electrostatic contribution to the free-energy density, and this generic expression agrees with those found in Landau and Lifshitz [40] and Stratton [57] when the results in those books are specialized to incompressible materials. The term $\boldsymbol{E} \otimes \boldsymbol{D}$ arises no matter what constitutive model is used for the polarization $\boldsymbol{P}(\boldsymbol{E})$, so long as the recipe (4) is used (together with $\boldsymbol{D}=\varepsilon_{0} \boldsymbol{E}+\boldsymbol{P}(\boldsymbol{E})$ ) to construct $W_{\mathrm{E}}$.

In the classical setting of a linear dielectric medium $\left(W_{\mathrm{E}}=-\frac{1}{2} \boldsymbol{D} \cdot \boldsymbol{E}, \boldsymbol{D}=\boldsymbol{\varepsilon} \boldsymbol{E}\right)$, the expression above coincides with the Minkowski stress tensor $\mathbf{T}_{M}$ in (39). In that case, the net electrostatic force density ( $\left.\operatorname{div} \mathbf{T}_{M}\right)$ consists of the Coulomb force on the free-charge distribution $\left(\rho_{\mathrm{f}} \boldsymbol{E}\right)$, the Kelvin force on the polarization $((\nabla \boldsymbol{E}) \boldsymbol{P})$, plus a term from the pressure $\left(-\frac{1}{2} \nabla(\boldsymbol{P} \cdot \boldsymbol{E})\right)$, which we have attempted to interpret in various ways (by analogy to the force of an electric field on a surface charge density on a conductor, and by the related notion of a "self-field correction"). The combined terms $(\nabla \boldsymbol{E}) \boldsymbol{P}-\frac{1}{2} \nabla(\boldsymbol{P} \cdot \boldsymbol{E})$ agree with expressions for the "ponderomotive force" found in the literature for linear dielectrics: $-\frac{1}{2} \varepsilon_{i j, k} E_{i} E_{j} e_{k}$ (in general), $-\frac{1}{2} E^{2} \nabla \varepsilon$ (if the medium is isotropic). While it is more common in continuum mechanics to handle pressure gradients separately from body forces, the combination of $(\nabla \boldsymbol{E}) \boldsymbol{P}$ with $-\frac{1}{2} \nabla(\boldsymbol{P} \cdot \boldsymbol{E})$ is necessary here to obtain agreement with these other expressions of ponderomotive force. The $O(1)$ nature of the electric self field associated with the polarization density in a shrinking volume element $|\Delta V| \rightarrow 0$ (as discussed in Sec.5.2.2 must play a role somehow in the net electric force on this density of dipoles, and the term $-\frac{1}{2} \nabla(\boldsymbol{P} \cdot \boldsymbol{E})$ seems to be one way to account for it.

We have highlighted the differences that occur when flexoelectric terms are included. These take us out of the realm of a linear dielectric medium and also introduce couplings between $\nabla \boldsymbol{n}$ and $\boldsymbol{E}$. While the overall framework of our development remains unchangedthe expressions for the body force, stress, force balance, body couple, couple stress, and 
torque balance maintain their same general forms-there are new contributions introduced by the flexoelectric terms. We have derived and presented expressions for these new contributions and have tried to provide some interpretation of them. Concerning the expression for the total stress tensor $\mathbf{T}$ above (and in (29)), we note that there are flexoelectric contributions to the term $-(\nabla \boldsymbol{n})^{T} \partial W / \partial \nabla \boldsymbol{n}$, in addition to contributions to the expressions for $W$ and $\boldsymbol{D}$. Flexoelectric effects are complicated and difficult to interpret in general: they involve couplings among $\boldsymbol{n}, \nabla \boldsymbol{n}$, and $\boldsymbol{E}$, and they have both forward and inverse influences (director distortion inducing polarization and contributions to electric fields, and electric fields encouraging director distortion so as to minimize the coupling term $-\boldsymbol{P}_{\mathrm{f}}(\boldsymbol{n}, \nabla \boldsymbol{n}) \cdot \boldsymbol{E}$ in the free-energy density).

Any force field that enters our system in the form $\boldsymbol{F}=-\partial W / \partial x$ is balanced, in equilibrium, by all or part of a related pressure gradient. This follows from the force balance

$$
\operatorname{div} \mathbf{T}-\frac{\partial W}{\partial x}=\mathbf{0}
$$

and the fact that $-W$ forms part of the thermodynamic pressure $p=p_{0}-W$ :

$$
\operatorname{div} \mathbf{T}=\operatorname{div}\left[\left(W-p_{0}\right) \mathbf{I}-(\nabla \boldsymbol{n})^{T} \frac{\partial W}{\partial \nabla \boldsymbol{n}}+\boldsymbol{E} \otimes \boldsymbol{D}\right]=\nabla W-\cdots=\frac{\partial W}{\partial x}+\cdots
$$

The typical case here is that of a gravitational field. Another case (which we have not discussed in this report) would be the body force associated with a prescribed inhomogeneous magnetic field $\boldsymbol{H}=\boldsymbol{H}(x)$ (see [55, Sec. 3.1.4] or [56, Sec. 2.4.2 Remark (ii)]). The same type of balance holds true in the "compatibility potential" approach with $\boldsymbol{F}=\partial \Psi / \partial x$ and $p=p_{0}+\Psi-W$ (which, as we have noted earlier, is equivalent to our approach with $-\Psi$ folded into $W$ ), and this was observed by Ericksen some time ago [15, Sec. III.A]. In a more general sense, something similar holds true in the absence of any such force field at all, in which case the force balance

$$
\operatorname{div} \mathbf{T}=\mathbf{0}, \text { with } \mathbf{T}=\left(W_{\mathrm{e}}-p_{0}\right) \mathbf{I}-(\nabla \boldsymbol{n})^{T} \frac{\partial W_{\mathrm{e}}}{\partial \nabla \boldsymbol{n}}
$$

gives

$$
\nabla W_{\mathrm{e}}-\operatorname{div}\left[(\nabla \boldsymbol{n})^{T} \frac{\partial W_{\mathrm{e}}}{\partial \nabla \boldsymbol{n}}\right]=\mathbf{0}
$$

the effective force density associated with the Ericksen stress (the negative of the term in brackets above) being balanced by the negative gradient of the pressure from the distortional elastic energy density. Electric fields seem to behave in a different way, as seen in Sec.5.2.3. There, even in the absence of flexoelectric terms and with no free-charge distribution, the fully simplified force balance 42 , reads

$$
\operatorname{div} \mathbf{T}_{\mathrm{e}}+(\nabla \boldsymbol{E}) \boldsymbol{P}-\frac{1}{2} \nabla(\boldsymbol{P} \cdot \boldsymbol{E})=\mathbf{0}
$$

with $\mathbf{T}_{\mathrm{e}}$ as defined in (39), and we see that a combination of forces from both distortional elasticity and electrostatics is needed to obtain an equilibrium balance of forces (a reflection of the coupling between $\boldsymbol{n}$ and $U$ ).

Somewhat related to the above is the observation that the stress tensor $\mathbf{T}$ and couple stress tensor $\mathbf{L}$ contain mechanical and electrostatic influences commingled. This is consistent with the modern point of view in the continuum mechanics of electromagnetic mechanical systems. As already suggested by Toupin [60, Sec. 1] (dealing with elastic dielectric solids): "Any division of energy, momentum, stress, and energy flux into electromagnetic 
and mechanical components is bound to be somewhat arbitrary, and it is fruitless to attempt an independent theory of either component." This appears to be the contemporary consensus view. It also appears that past attempts at such divisions have led to some of the confusions and controversies in this area [4,5,7]. As also observed in [60, Sec. 4], the field equations and boundary conditions are independent of any chosen decomposition into mechanical and electromagnetic components. There are several ways in which work can be done in a system such as ours-this includes displacing matter, rotating $\boldsymbol{n}$, rotating $\boldsymbol{P}$, and moving charge on/off the electrodes - and these are reflected in various ways in the expression for the free energy.

We have focused on the case in which the electric field comes from electrodes held at constant potential by a battery or voltage source, the common case for liquid crystal experiments and devices. In such a case, the appropriate pointwise contribution to the freeenergy density can be written $-\int_{0}^{E} \boldsymbol{D} \cdot \mathrm{d} \boldsymbol{E}$ and takes the form $-\frac{1}{2} \boldsymbol{D} \cdot \boldsymbol{E}$ if the medium is a linear dielectric. While the electric potential remains constant on the electrodes in such a system, the charge densities on the electrode surfaces will in general change in response to changes in the state of the system. The voltage source may have to push charge on or off the electrodes to maintain the constant potential, and this work is what gives rise to the negative sign in the electrostatic contribution to the free-energy density. In other analyses of electromagnetic field/matter interactions, one often finds developments involving electric fields that are assumed to arise from fixed charge sources (charge distributions that do not change). For such systems, the appropriate contribution to the free-energy density would be $\int_{0}^{D} \boldsymbol{E} \cdot \mathrm{d} \boldsymbol{D}$, taking the form $+\frac{1}{2} \boldsymbol{D} \cdot \boldsymbol{E}$ for a linear dielectric [33, Sec. 4.7], [40, Sec. 10], [57, Sec. 2.8]. The contrasting energetics of "constant charge" versus "constant potential" are discussed in [33, Sec. 4.7] and [40, Secs. 5, 10] in general, and in [2, Secs. 3.6.2, 3.6.3] and [12. Sec. 10.1] in the context of liquid crystals. For the equilibrium electrostatic equations, one would have boundary conditions on $\boldsymbol{D} \cdot \boldsymbol{\nu}$ in the case of fixed charge sources versus boundary conditions on $U$ in the case of fixed potential.

Thus we have followed through a program to determine what results when one treats the electric potential as a state variable of equal standing with the director field. While the overall analysis and approach have considerable overlap with earlier works, they provide generalizations and (we hope) remove some of the question marks about the differences in handling magnetic fields versus electric fields in settings such as these. We also hope that our findings can shed some light on electric field/matter interactions in other settings, at least in the circumstances of incompressible fluid hydrostatics. Electromagnetic stress in ponderable media is complicated and has a long history that includes a number of controversies. Here, without taking sides in any of these debates, we have shown what expressions result from our starting point (of a free-energy density that contains potentials for all force fields of external origin) and have observed how these expressions compare with others found in the literature. To extend these ideas to liquid crystal hydrodynamics (at this level of modeling), a reasonable approach would seem to be to treat the electric field as adjusting instantaneously to motions of the fluid and director field (by virtue of the large differences in the time scales for fluid motion and director orientation changes compared to dielectric relaxation times [50]). This would result in a PDE-constrained version of the Ericksen-Leslie equations (with appropriate terms incorporated into the free-energy density, such as those we have given in Sec.2.1.

Acknowledgements The author is grateful to E. G. Virga for helpful comments on an earlier draft of this report and to P. Palffy-Muhoray for helpful discussions concerning surface anchoring energies. 


\section{References}

1. Ball, J.M.: Mathematics and liquid crystals. Mol. Cryst. Liq. Cryst. 647(1), 1-27 (2017)

2. Barbero, G., Evangelista, L.R.: An Elementary Course on the Continuum Theory for Nematic Liquid Crystals. World Scientific, Singapore (2001)

3. Barbero, G., Evangelista, L.R.: Adsorption Phenomena and Anchoring Energy in Nematic Liquid Crystals. CRC Press, Boca Raton (2006)

4. Barnett, S.M.: Resolution of the Abraham-Minkowski dilemma. Phys. Rev. Lett. 104, 070401 (2010)

5. Barnett, S.M., Loudon, R.: The enigma of optical momentum in a medium. Phil. Trans. R. Soc. A 368, 927-939 (2010)

6. Barratt, P.J., Jenkins, J.T.: Interfacial effects in the magnetohydrostatic theory of nematic liquid crystals. J. Phys. A: Math. Nucl. Gen. 6, 756-769 (1973)

7. Böcker, J.: Some reflections on electromagnetic force in matter. In: 2016 International Symposium on Power Electronics, Electrical Drives, Automation and Motion (SPEEDAM), pp. 1433-1440. IEEE (2016)

8. Buka, A., Éber, N. (eds.): Flexoelectricity in Liquid Crystals: Theory, Experiments and Applications. Imperial College Press, London (2013)

9. Bustamante, R., Dorfmann, A., Ogden, R.W.: Nonlinear electroelastostatics: a variational framework. Z. Angew. Math. Phys. 60, 154-177 (2009)

10. Bustamante, R., Dorfmann, A., Ogden, R.W.: On electric body forces and Maxwell stresses in nonlinearly electroelastic solids. Int. J. Engr. Sci. 47, 1131-1141 (2009)

11. Callen, H.B.: Thermodynamics and an Introduction to Thermostatistics, 2nd edn. John Wiley \& Sons, New York (1985)

12. Collings, P.J., Hird, M.: Introduction to Liquid Crystals Chemistry and Physics. Taylor \& Francis, London (1997)

13. Ericksen, J.L.: Conservation laws for liquid crystals. Trans. Soc. Rheol. 5, 23-34 (1961)

14. Ericksen, J.L.: Hydrostatic theory of liquid crystals. Arch. Ration. Mech. Anal. 9(1), 371-378 (1962)

15. Ericksen, J.L.: Equilibrium theory of liquid crystals. In: G.H. Brown (ed.) Advances in Liquid Crystals, vol. 2, pp. 233-298. Academic Press, New York (1976)

16. Eringen, A.C.: Continuum theory of nematic liquid crystals subject to electromagnetic fields. J. Math. Phys. 20(12), 2671-2681 (1979)

17. Eringen, A.C., Maugin, G.A.: Electrodynamics of Continua I: Foundations and Solid Media. SpringerVerlag, New York (1990)

18. Eringen, A.C., Maugin, G.A.: Electrodynamics of Continua II: Fluids and Complex Media. SpringerVerlag, New York (1990)

19. Eu, B.C.: Statistical foundation of the Minkowski tensor for ponderable media. Phys. Rev. A 33(6), 4121-4131 (1986)

20. Eu, B.C., Oppenheim, I.: On the Minkowski tensor and thermodynamics of media in an electromagnetic field. Physica 136A, 233-254 (1986)

21. Faetti, S., Virga, E.G.: On a curvature surface energy for nematic liquid crystals. Arch. Rational Mech. Anal. 140(1), 31-52 (1997)

22. Frank, F.C.: On the theory of liquid crystals. Discuss. Faraday Soc. 25, 19-28 (1958)

23. Gelfand, I.M., Fomin, S.V.: Calculus of Variations. Prentice-Hall, Englewood Cliffs, N.J. (1963). Translated and Edited by Richard A. Silverman

24. de Gennes, P.G., Prost, J.: The Physics of Liquid Crystals, 2nd edn. Clarendon Press, Oxford (1993)

25. de Groot, S.R.: The Maxwell Equations. North Holland, Amsterdam (1969)

26. de Groot, S.R., Suttorp, L.G.: Foundations of Electrodynamics. North Holland, Amsterdam (1972)

27. Guggenheim, E.A.: Thermodynamics: An Advanced Treatment for Chemists and Physicists, 5th edn. North-Holland, Amsterdam (1967)

28. Guo, T., Zheng, X., Palffy-Muhoray, P.: Surface anchoring energy of cholesteric liquid crystals. Liquid Crystals (2019). DOI 10.1080/02678292.2019.1660425

29. Gurtin, M.E.: An Introduction to Continuum Mechanics. Academic Press, San Diego (1981)

30. Gurtin, M.E.: Multiphase thermomechanics with interfacial structure 1. Heat conduction and the capillary balance law. Arch. Rational Mech. Anal. 104(3), 195-221 (1988)

31. Gurtin, M.E., Murdoch, A.I.: A continuum theory of elastic material surfaces. Arch. Rational Mech. Anal. 57(4), 291-323 (1975)

32. Hutter, K., van de Ven, A.A.F., Ursescu, A.: Electromagnetic Field Matter Interactions in Thermoelastic Solids and Viscous Fluids. Springer, Berlin Heidelberg (2006)

33. Jackson, J.D.: Classical Electrodynamics, 2nd edn. John Wiley \& Sons, New York (1975)

34. Jákli, A., Saupe, A.: One- and Two-Dimensional Fluids: Properties of Smectic, Lamellar and Columnar Liquid Crystals. CRC Press, Boca Raton (2006) 
35. Jeffreys, S.H., Jeffreys), B.S.L.: Methods of Mathematical Physics, 3rd edn. Cambridge University Press, Cambridge (1956)

36. Jenkins, J.T., Barratt, P.J.: Interfacial effects in the static theory of nematic liquid crystals. Quart. J. Mech. Appl. Math. 27(1), 111-127 (1974)

37. Kellogg, O.D.: Foundations of Potential Theory. Springer, Berlin (1929)

38. Lagerwall, S.T.: Ferroelectric and Antiferroelectric Liquid Crystals. Wiley-VCH, Weinheim (1999)

39. Lanczos, C.: The Variational Principles of Mechanics, 4th edn. University of Toronto Press, Toronto (1970)

40. Landau, L.D., Lifshitz, E.M., Pitaevskii, L.P.: Electrodynamics of Continuous Media, 2nd edn. Butterworth-Heinemann, Oxford (1993)

41. Leslie, F.M.: Theory of flow phenomena in liquid crystals. In: G.H. Brown (ed.) Advances in Liquid Crystals, vol. 4, pp. 1-81. Academic Press, New York (1979)

42. Leslie, F.M.: Some topics in equilibrium theory of liquid crystals. In: J.L. Ericksen, D. Kinderlehrer (eds.) Theory and Applications of Liquid Crystals, IMA Volumes in Mathematics and Its Applications, vol. 5, pp. 211-234. Springer-Verlag, New York (1987)

43. Liu, L.: On energy formulations of electrostatics for continuum media. J. Mech. Phys. Solids 61, 968990 (2013)

44. Mansfield, E.L.: A Practical Guide to the Invariant Calculus. Cambridge University Press, Cambridge (2010)

45. McConnell, A.J.: Applications of Tensor Analysis. Dover, New York (1957)

46. Mottram, N.J., Newton, C.J.P.: Introduction to Q-tensor theory. arXiv.org e-Print archive (2014). URL https://arxiv.org/abs/1409.3542

47. Ogden, R.W.: Non-Linear Elastic Deformations. Dover, Mineola, New York (1997)

48. Oseen, C.W.: The theory of liquid crystals. Trans. Faraday Soc. 29, 883-889 (1933)

49. Reitz, J.R., Milford, F.J.: Foundations of Electromagnetic Theory, 2nd edn. Addison-Wesley, Reading, Massachusetts (1967)

50. Shiyanovskii, S.V., Lavrentovich, O.D.: Dielectric relaxation and memory effects in nematic liquid crystals. Liquid Crystals 37(6-7), 737-745 (2010)

51. Slattery, J.C., Sagis, L., Eun-Suok, O.: Interfacial Transport Phenomena, 2nd edn. Springer, New York (2007)

52. Sluckin, T.J.: Anchoring transitions at liquid crystal surfaces. Physica A 213, 105-109 (1995)

53. Sluckin, T.J., Poniewierski, A.: Orientational wetting transitions and related phenomena in nematics. In: C.A. Croxton (ed.) Fluid Interfacial Phenomena, chap. 5, pp. 215-253. John Wiley \& Sons, Chichester (1986)

54. Sonin, A.A.: The Surface Physics of Liquid Crystals. Gordon and Breach, Luxembourg (1995)

55. Sonnet, A.M., Virga, E.G.: Dissipative Ordered Fluids: Theories for Liquid Crystals. Springer, New York (2012)

56. Stewart, I.W.: The Static and Dynamic Continuum Theory of Liquid Crystals. Taylor \& Francis, London (2004)

57. Stratton, J.A.: Electromagnetic Theory. McGraw-Hill, New York and London (1941)

58. Tiersten, H.F.: A Development of the Equations of Electromagnetism in Material Continua. SpringerVerlag, New York (1990)

59. Toupin, R.A.: The elastic dielectric. J. Rational Mech. Anal. 5(6), 849-915 (1956)

60. Toupin, R.A.: Stress tensors in elastic dielectrics. Arch. Ration. Mech. Anal. 5(1), 440-452 (1960)

61. Truesdell, C., Noll, W.: The Non-Linear Field Theories of Mechanics, 3rd edn. Springer, Berlin and Heidelberg (2004)

62. Truesdell, C., Toupin, R.A.: The Classical Field Theories. In: S. Flügge (ed.) Principles of Classical Mechanics and Field Theory, Encyclopedia of Physics, vol. III/1, pp. 226-858. Springer-Verlag, Berlin Heidelberg (1960)

63. Virga, E.G.: Variational Theories for Liquid Crystals. Chapman \& Hall, London (1994)

64. Weatherburn, C.E.: Differential Geometry of Three Dimensions, vol. I. Cambridge University Press, Cambridge (1961)

65. Zocher, H.: The effect of a magnetic field on the nematic state. Trans. Faraday Soc. 29, 945-957 (1933) 